\title{
Módulos irredutíveis para subálgebras de Heisenberg de álgebras de Krichever-Novikov
}

Felipe Albino dos Santos

DisSERTAÇÃO APRESENTADA

$\mathrm{AO}$

Instituto DE MATEMÁtica E EstatísticA

DA

Universidade DE SÃo PAUlo

PARA

OBTENÇÃO DO TÍTULO

$\mathrm{DE}$

Mestre em CiênCIAS

Programa: Matemática

Orientador: Prof. Dr. Vyacheslav Futorny

Durante o desenvolvimento deste trabalho o autor recebeu auxílio financeiro do Conselho

Nacional de Desenvolvimento Científico e Tecnológico (Processo 133943/2015-1) e do Escritório Internacional Canadense para a Educação Internacional. 


\section{Módulos irredutíveis para subálgebras de Heisenberg de álgebras de Krichever-Novikov}

Esta versão da dissertação contém as correções e alterações sugeridas pela Comissão Julgadora durante a defesa da versão original do trabalho, realizada em 20/02/2017. Uma cópia da versão original está disponível no

Instituto de Matemática e Estatística da Universidade de São Paulo.

Comissão Julgadora:

- Prof. Dr. Vyacheslav Futorny (orientador) - IME-USP

- Prof. Dr. Viktor Bekkert - UFMG

- Prof. Dr. Luiz Enrique Ramirez - UFABC 


\section{Agradecimentos}

Durante os meus últimos anos de estudo, tive a grande oportunidade de contar com pessoas que cruzaram rapidamente o meu caminho de forma passageira, com pessoas que entraram definitivamente na minha vida pessoal e profissional e com pessoas que sempre estiveram e sempre estarão comigo. Todas essas pessoas contribuíram de forma fundamental e única neste trabalho e no meu crescimento pessoal.

Em primeiro lugar, agradeço à minha família pelo apoio material e emocional. Contar com o amparo incondicional da minha mãe, do meu pai e dos meus irmãos foi crucial para o bom desenvolvimento dos meus estudos.

Agradeço aos meus amigos que sempre me acompanharam durante todos os momentos. Tanto aqueles que estiveram nas ocasiões de lazer quanto os que me acompanharam na vida acadêmica tiveram grande impacto positivo na redação deste trabalho.

Agradeço ao meu orientador Vyacheslav Futorny pela sua orientação durante os últimos anos de trabalho juntos, pela proposta de trabalhar neste tema e pelas oportunidades de participar de atividades acadêmicas no Brasil e no exterior. Também agradeço ao professor Michael Lau que supervisionou meu estágio de pesquisa no Quebec e contribuiu significativamente para que os resultados apresentados aqui fossem alcançados.

Por último, mas não menos importante, expresso aqui um agradecimento especial ao meu amigo João Schwarz pelo suporte durante todo o meu mestrado e à minha psicóloga Márcia Neves pelos anos de acompanhamento. 


\section{Resumo}

DOS SANTOS, F. A. Módulos irredutíveis para subálgebras de Heisenberg de álgebras de Krichever-Novikov. 2017. 64 f. Dissertação (Mestrado) - Instituto de Matemática e Estatística, Universidade de São Paulo, São Paulo, 2017.

Este trabalho apresenta, de forma resumida, dois anos de estudo sob orientação do professor Vyacheslav Futorny. Durante este período, o autor pôde ter contato com a comunidade científica trabalhando em álgebras de Lie e suas representações, além de fazer suas primeiras contribuições originais nesta área. O autor também dedicou-se ao estudo de material preliminar e a um primeiro contato com artigos de pesquisa e publicações recentes. Entre fevereiro e julho de 2016, foi estagiário de pesquisa na Université Laval (Quebec) sob supervisão do professor Michael Lau, onde produziu parte significativa desta dissertação. Os principais resultados deste trabalho foram alcançados em colaboração com os professores Futorny e Lau. Esta dissertação oferece uma introdução às já conhecidas álgebras de Krichever-Novikov se restringindo aos exemplos abordados previamente em [Bre95], [Cox08], [CJ13],[CFM14] e [BCF09], e as definições de estruturas que podem auxiliar a estudar estes espaços, incluindo álgebras de Lie afins, álgebras de loop e módulos de Verma. Considerando $\mathscr{K}$ uma álgebra de Krichever-Novikov do tipo 4-ponto, 3-ponto, elíptica ou DJKM e suas respectivas

subálgebras de Heisenberg $\hat{\mathscr{K}}=\mathscr{K} \otimes \mathfrak{h}_{\mathscr{K}}$, onde $\mathfrak{h}_{\mathscr{K}}$ é a subálgebra de Cartan de $\mathscr{K}$, nos Teoremas 3.2.3, 3.4.3, 3.6.3 e 3.8.3 são apresentados critérios explícitos de irredutibilidade para $\hat{\mathscr{K}}$-módulos do tipo $\varphi$-Verma $M_{\hat{\mathscr{K}}, \varphi}$.

Palavras-chave: álgebras de Krichever-Novikov, álgebras de Heisenberg, módulos de Verma. 


\section{Abstract}

\section{DOS SANTOS, F. A. Representations of Heisenberg subálgebras of Krichever-}

Novikov algebras. 2017. 64 f. Dissertação (Mestrado) - Instituto de Matemática e Estatística, Universidade de São Paulo, São Paulo, 2017.

This work presents, in a summarized format, two years of study under the advisoring of professor Vyacheslav Futorny. During this period of time, the author was able to be in touch with the cientific comunity working in Lie algebras and their representations, and to do his first original contributions on Lie algebras as well. The author has also dedicated to the study of preliminary material and to a first contact with research articles and recent publications. From February to July, 2016, the author was a research intern at Université Laval (Québec) under the supervision of professor Michael Lau, and could produce a significant portion of this dissertation. The main results of this work were reached in collaboration with both Professor Futorny and Professor Lau. This work gives an introduction to the already known Krichever-Novikov algebras limited only to the examples approached before in [Bre95], [Cox08], [CJ13],[CFM14] and [BCF09], and the structures definitions that could help us to study these spaces, including affine Lie algebras, loop algebras and Verma modules. Let $\mathscr{K}$ be a 4-point, 3-point, elliptic or DJKM Krichever-Novikov algebra and its respective Heisenberg subalgebras $\hat{\mathscr{K}}=\mathscr{K} \otimes \mathfrak{h}_{\mathscr{K}}$, where $\mathfrak{h}_{\mathscr{K}}$ is the K Cartan subalgebra. In the Theorems 3.2.3, 3.4.3, 3.6.3 and 3.8.3 we will give a explicit irreducibility criteria for $\varphi$-Verma $\hat{\mathscr{K}}$-modules $M_{\hat{\mathscr{K}}, \varphi}$

Keywords: Krichever-Novikov algebras, Heisenberg algebras, Verma modules. 


\section{Sumário}

$\begin{array}{ll}\text { Lista de símbolos } & \text { ix }\end{array}$

$\mathbf{0}$ Introdução $\quad \mathbf{1}$

0.1 Sobre este trabalho . . . . . . . . . . . . . . . . . . . . . 1

0.2 Como este trabalho está organizado . . . . . . . . . . . . . . . . . 2

1 Conceitos básicos $\quad 5$

1.1 Álgebras de Lie afins . . . . . . . . . . . . . . . . . . . . . . . . 5 5

1.2 Álgebras de Heisenberg . . . . . . . . . . . . . . . . . . . . . . 7

1.3 O Teorema de Poincaré-Birkhoff-Witt $(\mathrm{PBW}) \ldots \ldots \ldots$

1.4 Álgebras de loop . . . . . . . . . . . . . . . . . . . 8

1.5 Decomposições triangulares . . . . . . . . . . . . . . . . . . . . . . 9

1.6 Módulos de Verma . . . . . . . . . . . . . . . . . . . . . . . . . . . . 10

1.7 Módulos irredutíveis para álgebras de Heisenberg e álgebras de Lie afins . . . 11

1.7.1 Módulos de Verma correspondentes à aplicação $\varphi$. . . . . . . . . . . 11

1.8 Módulos irredutíveis para álgebras de Heisenberg de uma extensão de álgebras de loop . . . . . . . . . . . . . . . . . . . . . . . . . 12

2 Ålgebras de Krichever-Novikov $\quad 15$

2.1 Extensão central universal de álgebra de Lie . . . . . . . . . . . . . . . . . . 15

2.2 Álgebra de Virasoro . . . . . . . . . . . . . . . . . . . . . . . 16

2.3 Álgebras de Krichever-Novikov . . . . . . . . . . . . . . . . . . . . . . 17

2.4 Módulo de diferenciais de Kähler . . . . . . . . . . . . . . . . . . . 17

2.5 Exemplos . . . . . . . . . . . . . . . . . . . 17

2.5.1 A álgebra de Lie 4-ponto $\mathscr{A} \ldots \ldots$. . . . . . . . . . . . . . . . . . . . . . . . . . . . . .

$2.5 .2 \quad$ A álgebra de Lie 3 -ponto $\mathscr{B} \ldots \ldots \ldots$

2.5.3 A álgebra de Lie elíptica $\mathscr{C}$. . . . . . . . . . . . . . . . . . . . 21

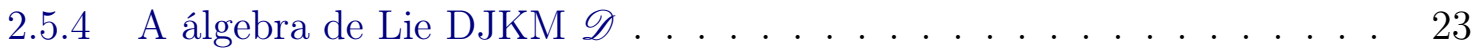

3 Módulos de Verma para álgebras de Krichever-Novikov 25

3.1 Módulos de Verma para $\hat{\mathscr{A}} \ldots \ldots \ldots \ldots$

3.2 Módulos $\varphi$-Verma para $\hat{\mathscr{A}} \ldots \ldots \ldots \ldots$ 
3.3 Módulos de Verma para $\hat{\mathscr{B}} \ldots \ldots \ldots \ldots$. . . . . . . . . . . . . 35

3.4 Módulos $\varphi$-Verma para $\hat{\mathscr{B}} \ldots \ldots \ldots \ldots$

3.5 Módulos de Verma para $\hat{\mathscr{C}} \ldots \ldots \ldots$. . . . . . . . . . . . . 43

3.6 Módulos $\varphi$-Verma para $\hat{\mathscr{C}} \ldots \ldots \ldots \ldots \ldots \ldots$

3.7 Módulos de Verma para $\hat{\mathscr{D}} \ldots \ldots \ldots$. . . . . . . . . . . . . 52

3.8 Módulos $\varphi$-Verma para $\hat{\mathscr{D}} \ldots \ldots \ldots \ldots \ldots$

$\begin{array}{ll}\text { Referências Bibliográficas } & 63\end{array}$ 


\section{Lista de símbolos}

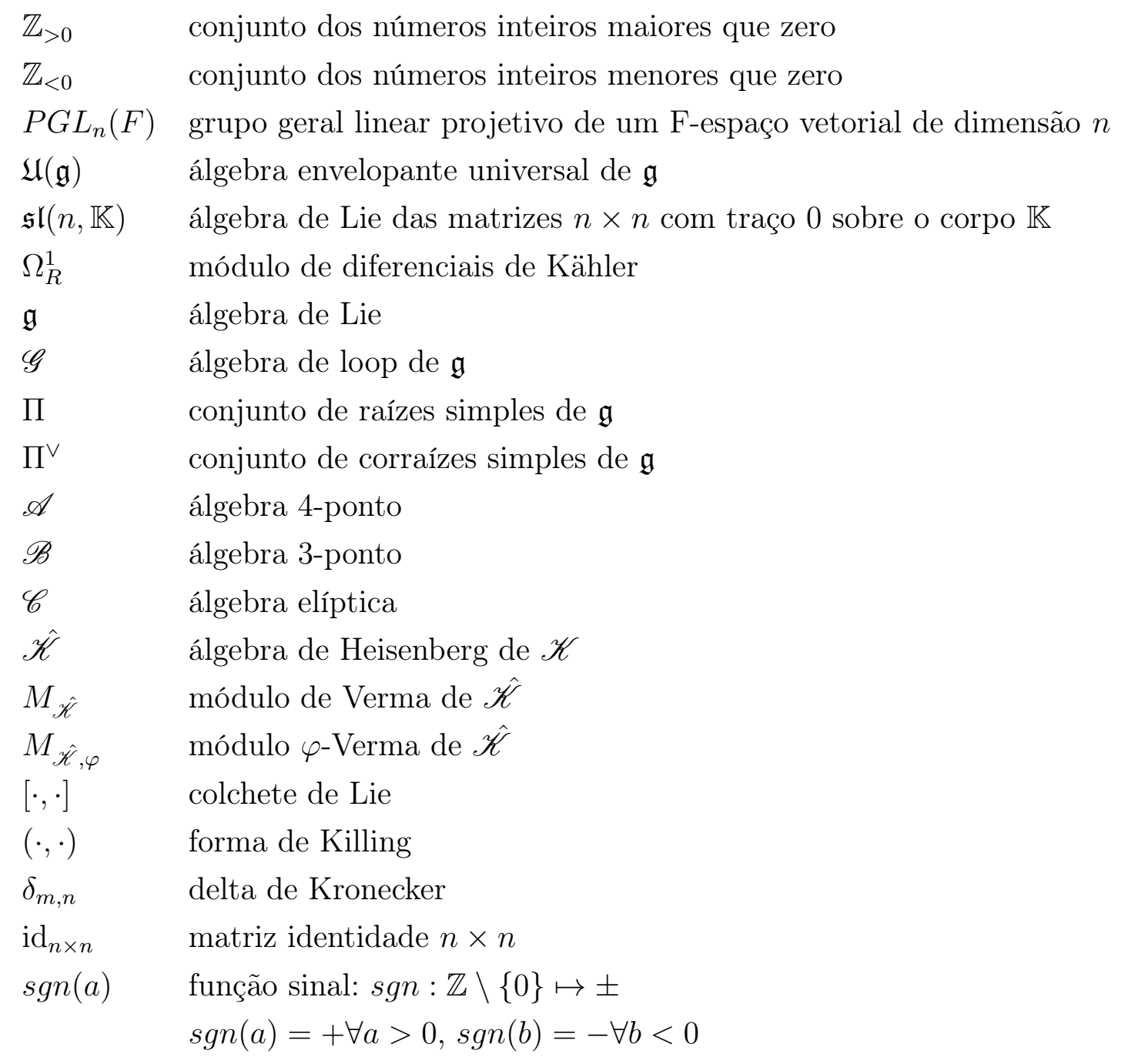




\section{Capítulo 0}

\section{Introdução}

\subsection{Sobre este trabalho}

Durante os últimos 45 anos, matemáticos e físicos estudam em cuidadoso detalhe as estruturas das álgebras de Kac-Moody. Se $\mathfrak{g}$ é uma álgebra de Lie complexa e de dimensão finita, a álgebra de loop de $\mathfrak{g}$ é a álgebra de Lie complexa de dimensão infinita $\mathscr{G}=\mathfrak{g} \otimes \mathbb{C}\left[t, t^{-1}\right]$ com colchete de Lie dado por $[x \otimes f, y \otimes g]=[x y] \otimes f g$, com $x, y \in \mathfrak{g}$. Chamamos $\hat{\mathscr{G}}$ de extensão central universal de $\mathscr{G}$ da álgebra de Kac-Moody afim não torcida.

Em 1995, Bremner introduziu em [Bre95] a ideia de generalizar a construção de álgebras de loop obtendo uma nova e interessante estrutura algébrica inspirado nas álgebras de Krichever-Novikov, em particular em [KN87]. Estes tipos de álgebras foram denominadas álgebras n-ponto. Apoiado especialmente num resultado sobre extensões centrais universais de álgebras de Lie em [Kas84], Bremner descreveu em sua publicação toda a estrutura das álgebras de Lie 4-ponto. Nos anos seguintes a esta publicação, alguns trabalhos foram publicados com o objetivo de compreender melhor possibilidades de generalizações de álgebras de loop, como podemos ver em [CJ13]. Além das álgebras n-ponto, outros exemplos de álgebras de Krichever-Novikov foram explorados, como as álgebras de Lie elípticas em [BCF09] e as álgebras de Lie DJKM em [CFM14].

Em 2008, em [Cox08] Cox publicou um estudo sobre o caso de álgebras 4-ponto de álgebras $\mathfrak{s l}(2, \mathbb{C})$. No ano seguinte, Cox, Bueno e Futorny publicam em [BCF09] outro estudo sobre um caso particular de álgebras de Krichever-Novikov, desta vez o caso de álgebras elípticas.

Em 2011, Bekkert, Benkart, Futorny e Kashuba publicam [BBFK13] apresentando novos módulos irredutíveis para álgebras de Heisenberg e álgebras de Lie afins. Neste trabalho, foi apresentada uma forma de generalizar módulos de tipo Verma denominados módulos $\varphi$-Verma. A construção é feita por meio de uma função $\varphi: \mathbb{N} \rightarrow\{ \pm\}$, com o objetivo de "misturar" a parte positiva e negativa da decomposição triangular de uma subálgebra de Heisenberg de uma álgebra de Lie afim. Foram explicitados critérios de irredutibilidade para estes módulos e este resultado pode ser aplicado em álgebras de loop de $\mathfrak{s l}(2, \mathbb{C})$.

Finalmente em 2013, Cox, Futorny e Martins publicam [CFM14], explicitando toda a estrutura das álgebras Date-Jimbo-Kashiwara-Miwa, mais um caso de álgebras de KricheverNovikov.

Tomando este histórico como ponto de partida, mostraremos que é possível, de forma similar a que [BBFK13] obtêm uma classificação para módulos de Verma e $\varphi$-Verma para subálgebras de Heisenberg de álgebras de Lie afins, obter módulos de Verma e $\varphi$-Verma irredutíveis para subálgebras de Heisenberg das álgebras 4-ponto, 3-ponto, elípticas e DateJimbo-Kashiwara-Miwa. A estratégia para a demonstração é muito parecida, porém algumas 
adaptações são necessárias.

A presente dissertação de mestrado apresenta o trabalho desenvolvido entre março de 2015 e dezembro de 2016, onde o autor teve contato pela primeira vez com artigos, a experiência de trabalhar com pesquisa relacionando diferentes artigos sob a orientação do professor Futorny, contato com resultados inéditos e a oportunidade enriquecedora de estagiar por seis meses no Quebec sob supervisão do professor Michael Lau.

Este trabalho alcança alguns resultados inéditos sobre a classificação de módulos irredutíveis para subálgebras de Heisenberg de álgebras de Krichever-Novikov, apresentados no Capítulo 3. Os principais resultados deste trabalho são:

1. Teorema 3.2.3: Seja $\mathscr{A}$ a álgebra de Lie 4-ponto dada segundo a Definição 2.5.5 e sua subálgebra de Heisenberg $\hat{\mathscr{A}}$ dada segundo a Definição 3.1.1. Considere o módulo $\varphi$-Verma $M_{\hat{\mathscr{A}}, \varphi}$ de $\hat{\mathscr{A}}$ conforme definido em 3.17. $M_{\hat{\mathscr{A}}, \varphi}$ é irredutível se, e somente se, nas relações $(3.16), \kappa_{0} \neq 0$ e $\chi_{+}=0$.

2. Teorema 3.4.3: Seja $\mathscr{B}$ a álgebra de Lie 3-ponto dada segundo a Definição 2.5.10 e sua subálgebra de Heisenberg $\hat{\mathscr{B}}$ dada segundo a Definição 3.3.1. Considere o módulo $\varphi$-Verma $M_{\hat{\mathscr{B}}, \varphi}$ de $\hat{\mathscr{B}}$ conforme definido em 3.40. $M_{\hat{\mathscr{B}}}, \varphi$ é irredutível se, e somente se, nas relações $(3.39), \kappa_{0} \neq 0$.

3. Teorema 3.6.3: Seja $\mathscr{C}$ a álgebra de Lie elíptica dada segundo a Definição 2.5.12 e sua subálgebra de Heisenberg $\hat{\mathscr{C}}$ dada segundo a Definição 3.5.1. Considere o módulo $\varphi$-Verma $M_{\hat{\mathscr{C}}, \varphi}$ de $\hat{\mathscr{C}}$ conforme definido em 3.69. $M_{\hat{\mathscr{C}}, \varphi}$ é irredutível se, e somente se, nas relações $(3.66), \kappa_{0} \neq 0$.

4. Teorema 3.8.3: Seja $\mathscr{D}$ a álgebra de Lie Date-Jimbo-Kashiwara-Miwa dada segundo a Definição 2.5.16 e sua subálgebra de Heisenberg $\hat{\mathscr{D}}$ dada segundo a Definição 3.7.1. Considere o módulo $\varphi$-Verma $M_{\hat{\mathscr{D}}, \varphi}$ de $\hat{\mathscr{D}}, \varphi$ conforme definido em 3.94. $M_{\hat{\mathscr{D}}, \varphi}$ é irredutível se, e somente se, nas relações $(3.93), \kappa_{0} \neq 0$.

Obtidos, respectivamente, como generalizações dos Teoremas 3.1.4, 3.3.4, 3.5.4 e 3.7.4, também alcançados neste trabalho.

\subsection{Como este trabalho está organizado}

Esta dissertação prevê um leitor com conhecimentos prévios sobre álgebras de Lie e suas representações e, por esse motivo, sugerimos ao longo do texto bibliografias auxiliares. Dito isso, apresentamos a forma como está organizada esta dissertação:

O Capítulo 1 é dedicado às estruturas fundamentais. Aqui o leitor encontrará as definições de álgebra de Lie afim, álgebra de Heisenberg, álgebra de loop e módulo de Verma. Na Seção 1.1 apresentamos uma motivação física e matemática para o estudo de álgebras de Lie afins. Apoiados principalmente em [Fuc95], construímos álgebras de Kac-Moody e álgebras de Lie afins. Na Seção 1.2 seguimos com a definição de álgebras de Heisenberg, estrutura fundamental para este trabalho, pois estudamos seus módulos irredutíveis no Capítulo 3. Na Seção 1.4 definimos álgebras de loop por meio de grupos de loop seguindo a construção de [DKBTK97], a generalização dessa construção é parte da motivação em [Bre95]. Na Seção 1.6 definimos rapidamente o conceito de vetor de peso, vetor de peso máximo e finalmente de módulo de Verma. Na Seção 1.7, destacamos os tópicos mais importantes para este trabalho obtidos em [BBFK13]. Definimos os módulos $\varphi$-Verma, que são uma generalização 
dos módulos de Verma da Seção 1.6, uma decomposição triangular de uma álgebra de Lie afim e um critério de irredutibilidade para módulos $\varphi$-Verma de subálgebras de Heisenberg de álgebras de Lie afim será apresentada. Finalmente, na Seção 1.8 exibiremos uma aplicação concreta do critério de irredutibilidade apresentado na seção anterior. De fato, exibiremos como o resultado de [BBFK13] pode ser aplicado numa extensão da álgebra de loop de $\mathfrak{s l}(2, \mathbb{C})$, confirmando o que Kac apresenta em [Kac94].

No Capítulo 2, introduzimos mais algumas definições relevantes neste trabalho. Na Seção 2.1, apoiados principalmente em [MP95], definimos a extensão central universal de uma álgebra de Lie e então justificamos o motivo pelo qual utilizamos determinada extensão na Seção 1.8. Na Seção 2.2, seguindo [Sch14], apresentamos uma muito breve motivação para álgebras de Virasoro, e a definimos como sendo a extensão central universal da álgebra de Witt, definida logo no início desta seção. Na Seção 2.3, damos a intuição geométrica para as álgebras de Krichever-Novikov. Aqui, optamos por não formalizar a construção destas estruturas para esquivarmos-nos dos detalhes talvez supérfluos para esta abordagem introdutória, no entanto, a construção detalhada pode ser apreciada em [Sch14] e fica como referência para o leitor motivado a entender profundamente este tópico. A Seção 2.5 mostra os exemplos principais de álgebras de Krichever-Novikov estudados durante este trabalho, são eles: a álgebra de Lie 4-ponto, estudada em [Bre95] e [Cox08], a álgebra de Lie 3-ponto estudada em [CJ13], a álgebra de Lie elíptica estudada em [BCF09] e a álgebra de Lie Date-Jimbo-Kashiwara-Miwa estudada em [CFM14].

O Capítulo 3 encerra este trabalho fazendo uso de todas as ferramentas abordadas previamente. Este capítulo contém resultados originais e é um primeiro passo para a produção de material novo em álgebras de Lie. Nele, classificamos quanto à irredutibilidade dos módulos de Verma e do tipo $\varphi$-Verma dos exemplos do Capítulo 2, utilizando técnicas similares às encontradas [BBFK13] com as devidas adaptações. 


\section{Capítulo 1}

\section{Conceitos básicos}

Neste capítulo nos apoiamos principalmente na abordagem do clássico livro de Kac intitulado Infinite-dimensional Lie algebras [Kac94] e no livro de Fuchs chamado Affine Lie Algebras and Quantum Groups [Fuc95] de forma a recordar rapidamente o que são álgebras de Lie afins, álgebras de loop, álgebras de Heisenberg e algumas outras estruturas fundamentais. Em seguida, apresentaremos alguns resultados obtidos em [BBFK13].

Ao leitor que não tem familiaridade com Teoria de Representações, recomendamos a consulta de [EGH $\left.{ }^{+} 11\right]$ e [Hum72]. Caso seja necessário revisitar algumas noções de álgebras de Lie, recomendamos [SM10].

\section{1 Álgebras de Lie afins}

Em física, álgebras de Lie se inserem na descrição de simetrias. Como alguns sistemas interessantes possuem infinitas simetrias, álgebras de Lie de dimensão infinita têm um importante papel. Uma classe interessante dessas estruturas é aquela que estuda álgebras de Lie de aplicações suaves de uma variedade para uma álgebra de Lie de dimensão finita. As álgebras de Kac-Moody são exemplos deste tipo de álgebra. Olhando mais de perto, as álgebras de Kac-Moody são de grande interesse pois uma relevante subclasse delas pode ser classificada completamente e sua teoria de representações é ainda mais rica do que aquela de álgebras de Lie simples de dimensão finita. Essa subclasse é a de álgebras de Lie afins.

Dada uma álgebra de Lie simples de dimensão finita $\mathfrak{g}$, lembre-se que podemos determinála completamente utilizando-nos das relações de Chevalley-Serre, isto é, por meio de $3 r$ geradores $\left\{e_{ \pm}^{i}, h^{i} \mid i=1, \ldots, r\right\}$ com colchetes obedecendo à identidade de Jacobi e às relações

$$
\begin{aligned}
& {\left[h^{i}, h^{j}\right]=0} \\
& {\left[h^{i}, e_{ \pm}^{j}\right]= \pm a_{j i} e_{ \pm}^{j}} \\
& {\left[e_{+}^{i}, e_{-}^{j}\right]=\delta_{i, j} h^{j}} \\
& \left(a d_{e_{ \pm}^{i}}\right)^{1-a_{j i}} e_{ \pm}^{j}=0 \text { para } i \neq j
\end{aligned}
$$

onde $a d$ é a aplicação adjunta e $\left(a d_{x}\right)^{n}$ denota $\underbrace{a d_{x} \circ a d_{x} \circ \cdots \circ a d_{x}}_{n \text { vezes }}$, A é a matriz de Cartan $r \times r$ associada às raízes simples $\Phi_{s}$ com entradas $a_{i j}$. Poderíamos então obter álgebras de Lie simples apenas requerendo algumas condições sobre a matriz de Cartan $A$. São elas:

(c.i) A matriz de Cartan $A$ deve ser indecomponível (ou irredutível). Isto significa que $n \tilde{a} o$ existe uma reordenação das entradas de $A$ que permita descrevê-la como uma matriz 
diagonal de blocos

$$
\left(\begin{array}{cc}
A_{1} & 0 \\
0 & A_{2}
\end{array}\right) \text {. }
$$

Além disso,

(c.ii)

$$
\begin{aligned}
& a_{i i}=2, \\
& a_{i j} \leq 0 \text { para } i \neq j, \\
& a_{i j}=0 \Leftrightarrow a_{j i}=0, \\
& a_{i j} \in \mathbb{Z}
\end{aligned}
$$

$\mathrm{e}$

$$
\operatorname{det} A>0
$$

As álgebras de Kac-Moody são obtidas enfraquecendo as condições acima sobre a matriz de Cartan $A$. Em particular, se removermos a condição (1.4), obtemos as álgebras de KacMoody em geral. No entanto, ao invés de simplesmente remover esta condição, pode ser interessante substituí-la por outra.

Considere substituir (1.4) por

$$
\operatorname{det} A_{\{i\}}>0, \text { para todo } i=0, \ldots, r
$$

onde $A_{\{i\}}$ denota a matriz obtida de $A$ com a remoção da $i$-ésima linha e coluna. Renumerando os geradores de Chevalley com $i=1, \ldots, r$, teremos que a condição (1.5) implica que $\operatorname{det} A \geq r$.

Definição 1.1.1 (Matriz de Cartan afim). Uma matriz obedecendo às desigualdades (1.5) é chamada semidefinita degenerada positiva. Uma matriz obedecendo às relações (1.3) é chamada matriz de Cartan generalizada. Uma matriz de Cartan generalizada que é semidefinita degenerada positiva é chamada matriz de Cartan afim.

Finalmente apresentamos a definição das álgebras que dão título a esta seção.

Definição 1.1.2 (Álgebras de Lie afins). As álgebras de Lie dadas por geradores e relações como em (1.1) com matriz de Cartan $A$ afim são chamadas álgebras de Lie afins.

Agora partiremos para outras definições essenciais. Os detalhes adicionais sobre a teoria de cada uma dessas estruturas pode ser encontrado em [MP95].

A partir daqui assumiremos que a álgebra de Lie $\mathfrak{g}$ sempre será do tipo afim, a menos de menção contrária.

Antes de continuarmos, seguindo Moody e Pianzola [MP95], podemos definir a forma de Killing em $\mathfrak{g}$.

Definição 1.1.3 (Forma de Killing). Seja $\mathfrak{g}$ uma álgebra de Lie complexa de dimensão finita. A forma de Killing $\kappa: \mathfrak{g} \times \mathfrak{g} \rightarrow \mathfrak{g}$ é definida como

$$
\kappa(x, y)=\operatorname{tr}[\operatorname{ad}(x) \quad a d(y)]
$$

para todo $x, y \in \mathfrak{g}$. Aqui ad denota a aplicação adjunta e $\operatorname{tr}$ o traço. 


\section{2 Álgebras de Heisenberg}

Suponha que $\mathfrak{a}$ é uma álgebra de Lie sobre $\mathbb{C}$ que possui um subespaço $\mathfrak{c}$ de dimensão 1 com as seguintes propriedades

$$
\begin{aligned}
{[\mathfrak{a}, \mathfrak{a}] } & =\mathfrak{c} \\
{[\mathfrak{a}, \mathfrak{c}] } & =(0)
\end{aligned}
$$

Considere $\{b\}$ uma base de $\mathfrak{c}$ e a seguinte aplicação bilinear antissimétrica $\psi$

$$
[x, y]=\psi(x, y) b \text { para todo } x, y \in \mathfrak{a} .
$$

Como c está no radical de $\psi$, podemos obter uma aplicação bilinear antissimétrica $\psi$ em $\mathfrak{a} / \mathfrak{c}$.

Apresentaremos o Teorema PBW que é importante ferramenta nas demonstrações contidas nesta dissertação.

\subsection{O Teorema de Poincaré-Birkhoff-Witt (PBW)}

Nesta seção apresentamos o teorema PBW que é de extrema importância na teoria de representações de álgebras de Lie. Ele nos mostra como construir uma base para $\mathfrak{U}(\mathfrak{g})$ dada uma base de $\mathfrak{g}$.

Teorema 1.3.1 (PBW). Seja $\mathfrak{g}$ uma álgebra de Lie com uma $\mathbb{K}$-base $\left\{x_{j} \mid j \in J\right\}$ indexada num conjunto totalmente ordenado $J$. Seja $\mathfrak{U}(\mathfrak{g})$ a álgebra envelopante universal de $\mathfrak{g}$ e $i$ a aplicaçao $\mathbb{K}$-linear canônica de $\mathfrak{g} \operatorname{em~} \mathfrak{U}(\mathfrak{g})$, então

$$
i\left(x_{j_{1}}\right) i\left(x_{j_{2}}\right) \cdots i\left(x_{j_{n}}\right)
$$

onde $j_{1} \leq j_{2} \leq \cdots \leq j_{n}, n \in \mathbb{Z}_{>0}$, junto com $1 \in \mathbb{K}$ forma uma base de $\mathfrak{U}(\mathfrak{g})$.

Aqui $i$ denota um homomorfismo de álgebras $i: \mathfrak{g} \mapsto \mathfrak{U}(\mathfrak{g})$ tal que

(i) $i([x, y])=i(x) i(y)-i(y) i(x)$, para todo $x, y \in \lambda$.

(ii) Dada uma álgebra associativa $A$ e qualquer homomorfismo de álgebras de Lie $f: \mathfrak{g} \rightarrow$ $\operatorname{Lie}(A)$ existe um único homomorfismo de álgebras $f^{\prime}: \mathfrak{U}(\mathfrak{g}) \mapsto A$ tal que o diagrama

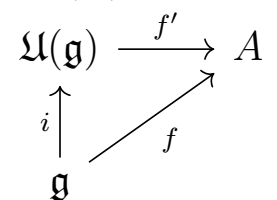

é comutativo.

Para demonstração e consequências, consultar [MP95].

Definição 1.3.2 (Álgebra de Heisenberg). Uma álgebra de Lie de Heisenberg, ou simplesmente álgebra de Heisenberg, é uma álgebra de Lie satisfazendo (1.6) e (1.7) com uma aplicação bilinear antissimétrica não degenerada $\psi$ em $\mathfrak{a} / \mathfrak{c}$.

Podemos também descrever uma álgebra de Heisenberg como sendo uma álgebra $\hat{\mathfrak{h}}$ gerada por $\left\{a_{n} \mid n \in \mathbb{Z} \backslash\{0\}\right\} \cup\{b\}$, onde $b$ é central e $\left[a_{m}, a_{n}\right]=m \delta_{m,-n} b$. 
Esta última maneira de descrever a álgebra de Heisenberg é a que parece mais familiar às estruturas que trabalhamos nos Exemplos 2.5. Em particular, toda álgebra de Lie afim possui uma álgebra de Heisenberg como subálgebra.

\section{4 Álgebras de loop}

Outra importante estrutura que gostaríamos de introduzir neste capítulo é a de álgebra de loop. Seguiremos exatamente a construção encontrada em [DKBTK97]. Álgebras de loop são álgebras de Lie associadas a grupos de loop.

Um grupo de loop é um grupo de aplicações de uma variedade $M$ para um grupo de Lie $G$. Trataremos em particular o caso em que $M$ é o círculo unitário e $G$ o grupo de Lie das matrizes $n \times n$.

Denotaremos $\tilde{G}$ o conjunto das aplicações suaves do círculo unitário $S^{1}$ para o grupo de Lie $G$, isto é, $\tilde{G}=\left\{\gamma \mid \gamma: S^{1} \rightarrow G\right\}$. Parametrizando $S^{1}$ com o ângulo $\theta$ teremos $\gamma: \theta \in S^{1} \mapsto$ $\gamma(\theta) \in G$, onde $\gamma(\theta)$ é uma matriz $n \times n$ cujas entradas são funções periódicas de $\theta$.

Proposição 1.4.1. O conjunto $\tilde{G}$ munido da multiplicação

$$
\left(\gamma_{1} \circ \gamma_{2}\right)(\theta):=\gamma_{1}(\theta) \gamma_{2}(\theta)
$$

possui uma estrutura de grupo. Este grupo é denominado grupo de loop.

Demonstraçãão. De fato (1.10) define uma aplicação associativa. Além disso, dado um elemento $\gamma \in \tilde{G}$, $\gamma$ tem um inverso denotado $\gamma^{-1}$ definido por

$$
\gamma^{-1}: \theta \in S^{1} \mapsto \gamma^{-1}(\theta):=\gamma(\theta)^{-1} \in G .
$$

Finalmente o elemento $\varepsilon \in \tilde{G}$ definido por

$$
\varepsilon: \theta \in S^{1} \mapsto \varepsilon(\theta)=e \in G
$$

(onde $e$ é a unidade em $G$ ) é a unidade em $\tilde{G}$.

Para obter a álgebra de Lie associada a $\tilde{G}$, consideraremos a aplicação $\sigma$ de um aberto $I=\{x \in \mathbb{R} \mid-\delta<x<\delta\} \subset \mathbb{R}$ no grupo $\tilde{G}$ com $\sigma(0)=\varepsilon \in \tilde{G}$. Este tipo de aplicação é chamada curva através da unidade de $\tilde{G}$. O vetor tangente à curva em $0 \in I$ é um elemento da álgebra de Lie do grupo de loop. Como $\sigma(s)$ é uma aplicação de $S^{1}$ para $\tilde{G}$, teremos

$$
\sigma(s): \theta \in S^{1} \mapsto \sigma(s)(\theta)
$$

onde $\sigma(s)(\theta)$ é uma matriz $n \times n$. Vamos adicionar a hipótese que as funções $\sigma$ são $C^{\infty}$.

Como $\sigma(s)$ passa por $s=0$, então $\sigma(0)(\theta)=\operatorname{Id}_{n \times n}$. Se fizermos a expansão de Taylor de $\sigma(t)(\theta)$ em volta de $s=0$ obtemos que

$$
\sigma(t)(\theta)=e+e \cdot h(\theta)+\cdots
$$

onde $h: \theta \in S^{1} \rightarrow h(\theta) \in L$ é uma aplicação suave de $S^{1}$ na álgebra de Lie $L$ associada a $G$. As entradas da matriz $h$ são elementos do tipo $h_{i j}=h_{i j}(\theta)$ do vetor tangente $h(\theta)=$ $\left.\frac{d}{d s} \sigma(s)(\theta)\right|_{s=0}$ à curva $\sigma(s)$ em $s=0$ e são definidos por $h_{i j}(\theta)=\left.\frac{d}{d s} \sigma_{i j}(s)(\theta)\right|_{s=0}$. O conjunto das aplicações $h: S^{1} \rightarrow L$ com o colchete

$$
\left[h_{1}, h_{2}\right](\theta):=h_{1}(\theta) h_{2}(\theta)-h_{2}(\theta) h_{1}(\theta)=\left[h_{1}(\theta), h_{2}(\theta)\right]
$$


é chamado de álgebra de loop. No entanto, podemos colocar ainda mais restrições sobre as funções $h$. Podemos, por exemplo, parametrizar o círculo $S^{1}$ por $t=e^{i \theta}$, o que nos restringiria às chamadas álgebras de loop polinomiais que neste trabalho, de forma a simplificar e seguir a nomenclatura dos artigos estudados, chamamos apenas de álgebras de loop. Abaixo vamos formalizar a definição de álgebra de loop adotada.

Definição 1.4.2 (Álgebra de loop). Seja $\mathfrak{g}$ uma álgebra de Lie complexa de dimensão finita. O subconjunto de aplicações $h: S^{1} \rightarrow \mathfrak{g}$

$$
\tilde{\mathfrak{g}}:=\left\{h \mid h(t)=\sum_{i=N}^{M} a_{i} t^{i}, a_{i} \in \mathfrak{g}, M, N \in \mathbb{Z}\right\}
$$

com colchete de Lie dado por

$$
\left[h_{1}, h_{2}\right](t):=\left[h_{1}(t), h_{2}(t)\right]
$$

é chamado de álgebra de loop.

A álgebra de Lie definida acima é isomorfa à álgebra de Lie

$$
\mathscr{G}=\mathfrak{g} \otimes \mathbb{C}\left[t, t^{-1}\right]
$$

com as relações de comutação dadas por

$$
[x \otimes f, y \otimes g]=[x, y] \otimes f g
$$

onde $x, y \in \mathfrak{g}$ e $f, g \in \mathbb{C}\left[t, t^{-1}\right]$. O anel $\mathbb{C}\left[t, t^{-1}\right]$ é o anel das funções racionais na esfera de Riemann com polos em $\{0, \infty\}$ e é conhecido como anel de polinômios de Laurent.

Esta última maneira de enxergarmos as álgebras de loop será muito útil, em particular nos Exemplos 2.5 do Capítulo 2.

\subsection{Decomposições triangulares}

Uma decomposição triangular de uma álgebra de Lie complexa $\mathfrak{g}$ consiste de uma subálgebra $\mathfrak{h} \neq(0)$ e duas subálgebras $\mathfrak{g}_{+}$e $\mathfrak{g}_{-}$tais que

(DT.1) $\mathfrak{g}=\mathfrak{g}_{-} \oplus \mathfrak{h} \oplus \mathfrak{g}_{+} ;$

(DT.2) $\mathfrak{g}_{+} \neq(0),\left[\mathfrak{h}, \mathfrak{g}_{+}\right] \subset \mathfrak{g}_{+}$, e $\mathfrak{g}_{+}$admite uma decomposição em espaços de peso relativa a $\mathfrak{h}$ com pesos $\alpha \neq 0$ chegando num semigrupo livre e aditivo $Q_{+} \subset \mathfrak{h}^{*}$;

(DT.3) existe uma anti-involução $\sigma$ (isto é, um antiautomorfismo de período 2) em $\mathfrak{g}$ tal que $\sigma\left(\mathfrak{g}_{+}\right)=\mathfrak{g}_{-}$e $\sigma_{\mid \mathfrak{h}}=\mathrm{id}_{\mid \mathfrak{h}}$

(DT.4) existe uma base $\left\{\alpha_{j}\right\}_{j \in J}$ de $Q_{+}$consistindo de elementos linearmente independentes de $\mathfrak{h}^{*}$. Em particular $Q_{+}$consiste de todas somas finitas não nulas da forma $\sum_{j \in J} m_{j} \alpha_{j}$, $m_{j} \in \mathbb{N}, J \subset \mathbb{N}$.

Segue abaixo um exemplo para ilustrar esta ideia. 


\section{Exemplo}

Se $\mathfrak{g}=\mathfrak{s l}(n, \mathbb{C}), n>1$, considere $\mathfrak{g}_{+}$a subálgebra das matrizes triangulares estritamente superiores e $\mathfrak{g}_{-}$a subálgebra das matrizes triangulares estritamente inferiores. Considere $\hat{\mathfrak{h}}$ a subálgebra das matrizes diagonais em $\mathfrak{g l}(n, \mathbb{C})$ e considere $\mathfrak{h}=\hat{\mathfrak{h}} \cap \mathfrak{s l}(n, \mathbb{C})$. Teremos que $\mathfrak{s l}(n, \mathbb{C})=\mathfrak{g}_{-} \oplus \mathfrak{h} \oplus \mathfrak{g}_{+}$é uma decomposição triangular de $\mathfrak{g}$.

\subsection{Módulos de Verma}

Considere que a álgebra de Lie $\mathfrak{g}$ possui decomposição triangular

$$
\mathfrak{g}=\mathfrak{g}_{-} \oplus \mathfrak{h} \oplus \mathfrak{g}_{+} \cdot
$$

Vamos relembrar rapidamente o que é um módulo de peso máximo.

Seja $\mathfrak{h}$ uma álgebra de Lie abeliana e $\pi: \mathfrak{h} \rightarrow \mathfrak{g l}(M)$ uma representação de $\mathfrak{h}$ num $\mathbb{K}$ espaço $M$. Considere $\phi \in \mathfrak{h}^{*}$. Um vetor $v \in M$ é chamado vetor de peso, com peso $\phi$, se para todo $h \in \mathfrak{h}$ tivermos que

$$
\pi(h) v=\phi(h) v .
$$

Considere $\pi$ uma representação de $\mathfrak{U}(\mathfrak{g})$ em $M$ e $v$ um vetor em $M$. O espaço dado por

$$
\pi(\mathfrak{U}(\mathfrak{g})) v:=\{\pi(u) v \mid u \in \mathfrak{U}(\mathfrak{g})\}
$$

é o menor submódulo de $M$ que contém $v$. Denominamos $\pi(\mathfrak{U}(\mathfrak{g})) v$ como submódulo de $M$ gerado por $v$.

Um vetor não nulo $v \in M$ é chamado vetor de peso máximo para $\mathfrak{g}$ se

(i) $v$ é um vetor de peso em relação à ação de sua álgebra de Cartan $\mathfrak{h}$.

(ii) $\pi(x) v=0$ para todo $x \in \mathfrak{g}_{+}$.

Um g-módulo $M$ é dito um módulo de peso máximo se $M$ é gerado por um vetor $v$ de peso máximo. O peso $\lambda \in \mathfrak{h}^{*}$ do vetor de peso máximo $v$ é chamado peso máximo de $M$ e chamamos o par $(\lambda, v)$ um par de peso máximo de $M$.

Seja $M$ um módulo de peso máximo com vetor de peso máximo $v$ e peso máximo $\lambda$. Suponha que $\mathfrak{g}_{-}$tem base $\left\{x_{j}\right\}_{j \in J}$ de vetores de raízes indexados num conjunto totalmente ordenado $J$. Para cada $j \in J$ denotaremos por $\gamma_{j}$ a raiz correspondente a $x_{j}$, isto é $x_{j} \in g_{\gamma_{j}}$. Sabemos que

$$
x_{i j}^{n_{k}} \cdots \cdots x_{i_{1}}^{n_{1}} \cdot v
$$

com $k \geq 0, i_{k}>i_{k-1}>\cdots>i_{1}, n_{j} \in \mathbb{Z}_{>0}$ é um vetor de peso de $\mathrm{M}$ de peso $\lambda+n_{1} \gamma_{1}+\cdots+n_{k} \gamma_{k}$ e esses vetores de peso geram como espaço vetorial $M$ chamado módulo de peso máximo.

Definição 1.6.1 (Módulo de Verma). Seja $\lambda \in \mathfrak{h}^{*}$. Um módulo de peso máximo $M(\lambda)$ para $\mathfrak{g}$ com peso máximo sendo o par $\left(\lambda, v_{+}\right)$é chamado módulo de Verma se para todo módulo de peso máximo $M$ com o par de peso máximo sendo $(\lambda, v)$ existe um homomorfismo de $\mathfrak{g}$-módulos $\eta: M(\lambda) \rightarrow M$ tal que $v_{+} \mapsto v$.

Exemplo: Módulos de Verma para álgebras de Heisenberg. Seja $\mathfrak{a}=\mathfrak{a}_{-} \oplus \mathbb{C} c \oplus \mathfrak{a}_{+}$uma álgebra de Heisenberg com uma anti-involução $\sigma$ e bases $\left\{a_{j}\right\},\left\{\sigma\left(a_{j}\right)\right\}(j \in \mathbb{Z})$ para $\mathfrak{a}_{+}$e $\mathfrak{a}_{-}$ tal que $\left[a_{j}, \sigma\left(a_{k}\right)\right]=\delta_{j k} c, k \in \mathbb{Z}$. 
Seja $\alpha \in \mathfrak{h}^{*}$ e $M(\lambda)$ o módulo de Verma para $\hat{\mathfrak{a}}$ com peso máximo sendo o par $\left(\lambda, v_{+}\right)$. Então $M(\lambda)=\bigoplus_{n \in \mathbb{N}} M(\lambda)^{\lambda-n \alpha}$ é a decomposição em espaços de peso correspondente de $M(\lambda)$. Além disso, se $\hat{A}$ é o aniquilador de $v_{+}$, então $M(\lambda)=\mathfrak{U}\left(\mathfrak{a}_{-}\right) \cdot v_{+} \hat{A}=\mathfrak{U}\left(\mathfrak{a}_{-}\right) \cdot v_{+}$. Detalhes e mais exemplos podem ser encontrados em [MP95].

\subsection{Módulos irredutíveis para álgebras de Heisenberg e álgebras de Lie afins}

Nesta seção, seguindo [BBFK13], destacaremos os principais resultados que serviram de motivação para o Capítulo 3.

\subsubsection{Módulos de Verma correspondentes à aplicação $\varphi$}

Seguindo [Kac94] sabemos que $\mathfrak{g}$ tem o que é chamado de decomposição em espaços de raízes em relação a $\mathfrak{h}$ (sua subálgebra de Cartan). Isto significa que podemos descrever $\mathfrak{g}$ como

$$
\mathfrak{g}=\mathfrak{h} \oplus \bigoplus_{\alpha \in \mathfrak{h}^{*} \backslash\{0\}} \mathfrak{g}_{\alpha}
$$

onde $\mathfrak{g}_{\alpha}=\{x \in \mathfrak{g} \mid[h, x]=\alpha(h) x, \forall h \in \mathfrak{h}\}$. Apresentaremos a seguir uma forma alternativa à Seção 1.5 de se definir uma decomposição triangular de uma álgebra de Lie.

Chamaremos o conjunto $\Delta=\left\{\alpha \in \mathfrak{h}^{*} \mid \mathfrak{g}_{\alpha} \neq(0)\right\}$ de reticulado das raízes de $\mathfrak{g}$ e este último tem uma partição $\Delta=\Delta_{+} \cup \Delta_{-}$em raízes positivas e negativas, onde $\Delta_{-}=-\Delta_{+}$. Se $S \subseteq \Delta$, diremos que $S$ admite uma partição, se $S \cup(-S)=\Delta$ e $S \cap(-S)=\emptyset$. Uma partição $\Delta=S \cup(-S)$ é dita uma partição fechada se sempre que $\alpha, \beta \in S$ e $\alpha+\beta \in \Delta$ tivermos que $\alpha+\beta \in S$. Para qualquer $S$ determinando uma partição de $\Delta$, os espaços $\mathfrak{g}_{S}=\oplus_{\alpha \in S} \mathfrak{g}_{\alpha}$ e $\mathfrak{g}_{-S}=\oplus_{\alpha \in-S} \mathfrak{g}_{\alpha}$ são subálgebras de $\mathfrak{g}$ e $\mathfrak{g}=\mathfrak{g}_{-S} \oplus \mathfrak{h} \oplus \mathfrak{g}_{S}$ é uma decomposição triangular de $\mathfrak{g}$.

Sabemos que se $A=\left(a_{i j}\right)_{i, j=1}^{n}$ é a matriz de Cartan associada, podemos construir um diagrama de Dynkin de $A$ (detalhes podem ser verificados no Capítulo 4 de [Kac94]). A partir deste diagrama, $\delta=\sum_{i=0}^{l} a_{i} \alpha_{i}$ onde os $a_{i}$ 's são exatamente as etiquetas dos vértices e $\alpha_{i}$ são os elementos da base de raízes de $\mathfrak{g}$. Chamamos $\delta$ de raiz imaginária indivisível.

Se $\mathfrak{h}$ é subálgebra de Cartan de $\mathfrak{g}$, considere $\Pi=\left\{\alpha_{0}, \ldots, \alpha_{l}\right\}$ o seu conjunto de raízes simples e $\Pi^{\vee}=\left\{\alpha_{0}^{\vee}, \ldots, \alpha_{l}^{\vee}\right\}$ o seu conjunto de corraízes simples. O elemento

$$
c=\sum_{i=0}^{l} a_{i}^{\vee} \alpha_{i}^{\vee}
$$

é chamado elemento central canônico e até o final deste capítulo será denotado simplesmente como $c$.

Tomando $\Delta_{+}$o espaço das raízes positivas do sistema de raízes de $\mathfrak{g}$, podemos descrevêlo como $\Delta_{+}=\Delta_{+}^{i m} \cup \Delta_{-}^{r e}$, sendo $\Delta_{+}^{i m}$ o espaço das raízes imaginárias, sabemos que $\Delta_{+}^{i m}=$ $\left\{n \delta \mid n \ni \mathbb{Z}_{>0}\right\}$. O subespaço $L:=\mathbb{C} c \oplus \bigoplus_{n \in \mathbb{Z} \backslash\{0\}} \mathfrak{g}_{n \delta}$ forma uma subálgebra de Heisenberg da álgebra $\mathfrak{g}$. Então, $[x, y]=\zeta(x, y) c$ para $x \in \mathfrak{g}_{m \delta}, y \in \mathfrak{g}_{n \delta}$, onde $\zeta(x, y)$ é uma forma bilinear antissimétrica com $\zeta\left(\mathfrak{g}_{m \delta}, \mathfrak{g}_{n \delta}\right)=0$ para $n \neq-m$ e cuja restrição para $\mathfrak{g}_{m \delta} \times \mathfrak{g}_{n \delta}$ é não degenerada para todo $m \neq 0$. 
A ideia aqui é seguir a Seção 3.1 de [BBFK13] onde é apresentada uma generalização na construção dos módulos de Verma correspondentes a uma função $\varphi$.

Podemos fazer a decomposição triangular de $L$ de forma que $L^{-} \oplus \mathbb{C} c \oplus L^{+}$, onde $L^{ \pm}=$ $\bigoplus_{n \in \mathbb{N}} \mathfrak{g}_{ \pm n \delta}$. Considere uma função $\varphi: \mathbb{N} \rightarrow\{ \pm\}$ e defina os espaços

$$
L_{\varphi}^{ \pm}=\left(\bigoplus_{n \in \mathbb{N}, \varphi(n)= \pm} \mathfrak{g}_{n \delta}\right) \oplus\left(\bigoplus_{m \in \mathbb{N}, \varphi(m)=\mp} \mathfrak{g}_{-m \delta}\right) .
$$

Estes espaços são subálgebras abelianas de $L$ e

$$
L=L_{\varphi}^{-} \oplus \mathbb{C} c \oplus L_{\varphi}^{+}
$$

é uma decomposição triangular de $L$.

Se $\mathbb{C} \nu$ é uma representação de dimensão 1 de $\mathbb{C} c \oplus L_{\varphi}^{+}$com $c \nu=a \nu$ para algum escalar $a \in \mathbb{C}$ e $L_{\varphi}^{+} \nu=0$, definimos o módulo de $\varphi$-Verma induzido por

$$
M_{\varphi}(a)=\mathfrak{U}(L) \otimes_{\mathfrak{U}\left(\mathbb{C} c \otimes L_{\varphi}^{+}\right)} \mathbb{C} \nu
$$

Não é difícil perceber que quando $\varphi(n)=+, \forall n \in \mathbb{N}$, teremos a decomposição triangular usual de $L$ em (1.21) e o módulo de Verma de $L$ em (1.23).

Finalmente alcançamos um resultado de [BBFK13] sobre a classificação destes módulos quanto a sua irredutibilidade.

Proposição 1.7.1. $M_{\varphi}(a)$ é irredutível se, e somente se, $a \neq 0$.

Demonstração. A estratégia para a prova começa definindo uma base de vetores raízes para $L_{\varphi}^{+}$e para $L_{\varphi}^{-}$. Após isso, considera-se elementos de $L_{\varphi}^{+}$e $L_{\varphi}^{-}$e supondo $a \neq 0$ percebe-se que aplicar um elemento da parte positiva num elemento da parte negativa reduz o grau da parte negativa em uma unidade. Isto nos mostra que quando $a \neq 0$, o submódulo gerado por $w$ contém $v$ e então é todo $M_{\varphi}(a)$. Por outro lado quando $a=0$, é fácil construir um submódulo próprio já que os elementos em $L_{\varphi}^{+}$sempre atuam como zero em $L_{\varphi}^{-}$.

A prova completa com detalhes pode ser apreciada no artigo [BBFK13] que publicou este resultado.

Este resultado é central para o desenvolvimento do Capítulo 3. Lá, aplicamos técnica similar àquela elaborada para a demonstração da proposição acima em casos particulares de álgebras de Krichever-Novikov.

\subsection{Módulos irredutíveis para álgebras de Heisenberg de uma extensão de álgebras de loop}

Parte da motivação para o estudo de álgebras n-ponto, definidas no Capítulo 2, é a generalização da construção de álgebras de loop. Vamos estudar agora como a Proposição 1.7.1 pode ser aplicada nas álgebras de loop de $\mathfrak{s l}(2, \mathbb{C})$ de forma a obter módulos de $\varphi$-Verma irredutíveis. Este é um resultado já conhecido e também pode ser observado em [Kac94]. Seja $\mathscr{G}$ a álgebra de loop de $\mathfrak{s l}(2, \mathbb{C})$ definida por $\mathscr{G}=\mathfrak{s l}(2, \mathbb{C}) \otimes \mathbb{C}\left[t, t^{-1}\right]$. Consideraremos uma extensão desta álgebra, $\hat{\mathscr{G}}=\mathfrak{s l}(2, \mathbb{C}) \otimes \mathbb{C}\left[t, t^{-1}\right] \oplus \mathbb{C} c$, onde $\mathbb{C} c$ é uma álgebra de Lie de dimensão 1 e abeliana. O colchete de $\hat{\mathscr{G}}$ é dado por

$$
[f(t) \otimes x+\mu c, g(t) \otimes y+\nu c]=f(t) g(t) \otimes[x, y]_{\mathfrak{g}}+\phi(f(t) \otimes x, g(t) \otimes y) c
$$


onde $\phi$ é uma função $\phi: \mathscr{G} \times \mathscr{G} \rightarrow \mathbb{C}$ tal que

$$
\phi(f(t) \otimes x, g(t) \otimes y):=\left(t \frac{d}{d t} f(t) \otimes x, g(t) \otimes y\right)
$$

para $f, g \in \mathbb{C}\left[t, t^{-1}\right]$ e $x, y \in \mathfrak{s l}(2, \mathbb{C})$.

Detalharemos o motivo de estudarmos este tipo de extensão no próximo capítulo.

A subálgebra de Heisenberg de $\hat{\mathscr{G}}$ é $L:=\mathfrak{h} \otimes \mathbb{C}\left[t, t^{-1}\right] \oplus \mathbb{C} c$, onde $\mathfrak{h}$ é a subálgebra de Cartan de $\mathfrak{s l}(2, \mathbb{C})$. Considere a seguinte álgebra de Heisenberg dada pelo espaço $H=$ $\mathbb{C} c \oplus \bigoplus_{i \in \mathbb{Z} \backslash\{0\}} \mathbb{C} e_{i}$, onde $\left[e_{i}, e_{j}\right]=\delta_{i,-j} c$, e $\left[e_{j}, c\right]=0$ para todo $i \geq 1$ e todo $j$. A subálgebra de Heisenberg $L$, da Seção 1.7.1, pode ser descrita como $H$ escolhendo uma base ortogonal em cada espaço de raízes $g_{k \delta}$ e uma base dual em $\mathfrak{g}_{-k \delta}$ para cada $j \geq 1$.

Sabemos que $\mathfrak{h}$, a álgebra de Cartan de $\mathfrak{s l}(2, \mathbb{C})$, tem dimensão 1. Seja

$$
\varphi:\left(\mathfrak{h} \otimes C\left[t, t^{-1}\right] \oplus \mathbb{C} c\right) \mapsto\left(\mathbb{C} c \oplus \bigoplus_{i \in \mathbb{Z} \backslash\{0\}} \mathbb{C} e_{i}\right)
$$

aplicação linear tal que

$$
\varphi\left(h t^{n}+a\right)=a+e_{n}
$$

onde $h \in \mathfrak{h}$ e $a \in \mathbb{C} c$. Como $\varphi$ define um isomorfismo, podemos diretamente aplicar a Proposição 1.7.1 obtendo assim módulos de Verma e $\varphi$-Verma irredutíveis para essa extensão de álgebra de loop para $\mathfrak{s l}(2, \mathbb{C})$. 


\section{Capítulo 2}

\section{Álgebras de Krichever-Novikov}

As álgebras de Krichever-Novikov são álgebras de Lie de dimensão infinita formadas por objetos meromorfos numa superfície de Riemann que são holomorfos exceto num número finito de pontos. Além do ponto de vista algébrico, interpretação geométrica das álgebras de Krichever-Novikov pode nos dar informações úteis no contexto geométrico.

\subsection{Extensão central universal de álgebra de Lie}

Do ponto de vista da Teoria de Representações, a extensão central universal de uma álgebra de Lie pode ser mais rica e interessante que a álgebra de Lie original. Para entender que estrutura é essa, vamos seguir [MP95].

Basicamente, uma álgebra de Lie $\mathfrak{e}$ é uma extensão central de uma álgebra de Lie $\mathfrak{g}$ se $\mathfrak{e}$ possui uma subálgebra $\mathfrak{c}$ contida em seu centro, tal que $\mathfrak{e} / \mathfrak{c} \cong \mathfrak{g}$. Um exemplo muito simples de extensão central é o caso em que $\mathfrak{e}=\mathfrak{g} \times \mathfrak{c}$ onde $\mathfrak{c}$ é uma álgebra de Lie abeliana. Para definir quando uma extensão central é universal, necessitaremos do conceito de cobertura.

Definição 2.1.1 (Extensão central). Vamos entender por uma extensão central de uma álgebra de Lie $\mathfrak{g}$ por $\mathfrak{c}$ uma sequência exata de álgebras de Lie

$$
0 \rightarrow \mathfrak{c} \stackrel{i}{\rightarrow} \mathfrak{e} \stackrel{\pi}{\rightarrow} \mathfrak{g} \rightarrow 0
$$

onde $\mathfrak{c}$ é o centro de $\mathfrak{e}$.

Dada outra extensão central

$$
0 \rightarrow \mathfrak{c}^{\prime} \stackrel{i^{\prime}}{\rightarrow} \mathfrak{e}^{\prime} \stackrel{\pi^{\prime}}{\rightarrow} \mathfrak{g} \rightarrow 0
$$

de $\mathfrak{g}$, diremos que há um morfismo entre (CE) e (CE') se existir um par $\left(\Phi, \Phi_{0}\right)$ de homomorfismos de álgebras de Lie de forma que o diagrama

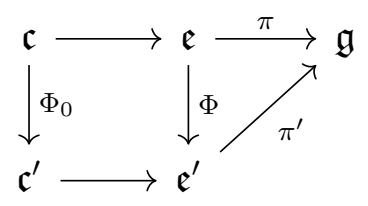

é comutativo.

Lembre-se: Uma álgebra de Lie $\mathfrak{I}$ é chamada perfeita se $\mathfrak{I}$ é igual sua álgebra derivada, isto é, se $\mathfrak{I}=[\mathfrak{I}, \mathfrak{I}]$. 
Definição 2.1.2 (Cobertura). A extensão central (CE) é dita uma cobertura de $\mathfrak{g}$ caso $\mathfrak{e}$ é perfeita.

Agora estamos prontos para definir extensão central universal.

Definição 2.1.3 (Extensão central universal). Diremos que uma cobertura de $\mathfrak{g}$ é uma extensão central universal se para toda extensão central de $\mathfrak{g}$, existir um único morfismo da cobertura para a extensão central.

A álgebra de loop $\mathscr{G}=\mathfrak{g} \otimes \mathbb{C}\left[t, t^{-1}\right]$ tem como extensão central universal $\hat{\mathscr{G}}=\mathfrak{g} \otimes \mathbb{C}\left[t, t^{-1}\right] \oplus$ $\mathbb{C} c$, onde $\mathbb{C} c$ é uma álgebra de Lie de dimensão 1 e abeliana. Isto justifica a escolha dessa extensão na Seção 1.8 .

Podemos, a seguir, definir a álgebra de Virasoro.

\section{2 Álgebra de Virasoro}

Sabemos que a álgebra de Virasoro aparece em matemática, em física matemática, em física teórica e em outros campos. Álgebras de Krichever-Novikov podem ser úteis para, de forma natural, generalizar a álgebra de Virasoro.

Nesta seção o objetivo é definir a álgebra de Virasoro de forma a oferecer motivações suficientes de sua generalização por meio de álgebras de Krichever-Novikov.

Definição 2.2.1 (Álgebra de Witt). Uma álgebra de Witt, denotada $\mathcal{W}$, é uma álgebra de Lie formada pelo espaço vetorial de base $\left\{e_{n} \mid n \in \mathbb{Z}\right\}$ com colchete de Lie dado por

$$
\left[e_{n}, e_{m}\right]=(m-n) e_{n+m}
$$

para $n, m \in \mathbb{Z}$.

Se definirmos $\operatorname{deg}\left(e_{n}\right):=n$, teremos que, de fato, $\mathcal{W}$ é uma álgebra graduada

$$
\mathcal{W}=\bigoplus_{n \in \mathbb{Z}} \mathcal{W}_{n}
$$

onde $\mathcal{W}_{n}=\left\langle e_{n}\right\rangle_{\mathbb{C}}$. Podemos descrever $\mathcal{W}$ como a álgebra formada pelas derivações da álgebra dos polinômios de Laurent $\mathbb{C}\left[t, t^{-1}\right]$.

A álgebra de Virasoro será a extensão central universal da álgebra de Witt.

Definição 2.2.2 (Álgebra de Virasoro). A álgebra de Virasoro $\mathcal{V}$ é a extensão central universal da álgebra de Witt. Uma álgebra de Virasoro é uma álgebra de Lie formada pelo espaço vetorial $\mathcal{V}=\mathbb{C} \oplus \mathcal{W}$. Dado $x \in \mathcal{W}$, se considerarmos $\hat{x}:=(0, x)$ e $t:=(1,0)$ uma base para $\mathcal{V}$ é $\hat{e}_{n}, n \in \mathbb{Z}$ e $t$, onde $\left\{e_{n} \mid n \in \mathbb{Z}\right\}$ é uma base para a álgebra de Witt. A álgebra de Virasoro será dada pelo espaço vetorial $\mathcal{V}$ com o colchete de Lie dado por

$$
\begin{aligned}
{\left[\hat{e}_{n}, \hat{e}_{m}\right] } & =(m-n) \hat{e}_{n+m}+\frac{1}{12}\left(n^{3}-n\right) \delta_{n+m, 0} t \\
{\left[\hat{e}_{n}, t\right] } & =[t, t]=0
\end{aligned}
$$

para todo $n, m \in \mathbb{Z}$. 
De forma similar àquela feita com a álgebra de Witt, podemos graduar $\mathcal{V}$ fazendo $\operatorname{deg}\left(\hat{e}_{n}\right)=\operatorname{deg}\left(e_{n}\right)=n$ e $\operatorname{deg}(t)=0$. A grosso modo, a álgebra de Virasoro é simplesmente a complexificação da álgebra de Lie $\operatorname{Vec}\left(S^{1}\right)$ dos campos de vetores no círculo $S^{1}$.

\section{3 Álgebras de Krichever-Novikov}

A álgebra de Virasoro é formada por objetos definidos na esfera de Riemann com no máximo dois polos fixos. No entanto, para determinadas aplicações em matemática e física isso não é o suficiente. Objetivando considerar mais que dois polos e superfícies de Riemann em geral, não apenas esferas de Riemann, aparecem as álgebras de Krichever-Novikov em [KN87], no ano de 1987.

Ao invés de uma definição formal, apresentamos aqui apenas uma ideia do que são as álgebras de Krichever-Novikov. Estas álgebras são dadas por meio de objetos meromorfos em superfícies de Riemann compactas de genus arbitrário e um comportamento "comportado"de polos. Isto significa que os polos serão permitidos apenas num número fixo.

Antes de citar alguns exemplos de álgebras de Krichever-Novikov, é fundamental a definição do módulo de diferenciais de Kähler. Então, primeiramente, dedicaremos algumas linhas para introduzir essa estrutura.

\subsection{Módulo de diferenciais de Kähler}

Proposição 2.4.1. Dado um anel comutativo $R$, existe um $R$-módulo $\Omega_{R}^{1}$ e uma derivação $d: R \rightarrow \Omega_{R}^{1}$ satisfazendo a seguinte propriedade universal: para todo $R$-módulo $M$ e uma derivação $\alpha: R \rightarrow M$, existe uma única aplicação $\Phi: \Omega_{R}^{1} \rightarrow M$ tal que o diagrama

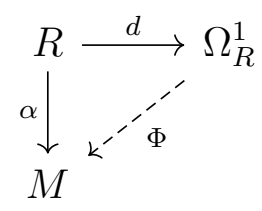

é comutativo. O módulo $\Omega_{R}^{1}$ é chamado de módulo de diferenciais de Kähler.

Nos exemplos que seguem utilizaremos o módulo destes diferenciais quocientado pelo espaço das diferenciais exatas. Podemos construir este espaço $\Omega_{R}^{1} / d R$ da seguinte maneira: seja $F=R \otimes R$ um $R$-módulo à esquerda com ação dada por $f(g \otimes h)=f g \otimes h$ para $f, g, h \in R$ e $K$ o submódulo de $F$ gerado por $1 \otimes f g-f \otimes g-g \otimes f$. Teremos que $F / K=\Omega_{R}^{1}$. Vamos denotar um elemento $f \otimes g+K$ de $\Omega_{R}^{1}$ por $f d g$. Definiremos uma aplicação $d: R \rightarrow \Omega_{R}^{1}$ por $d(f)=1 \otimes f+K$. As diferenciais exatas estão no subespaço $d R=d(R)$ e nós denotaremos a coclasse de $f d g$ módulo $d R$ por $\overline{f d g}$.

\subsection{Exemplos}

Nesta seção vamos destacar alguns exemplos de álgebras de Krichever-Novikov que foram estudados durante a elaboração deste trabalho. Em todos os exemplos tratados, estudamos as álgebras de Krichever-Novikov formadas por funções meromorfas em uma superfície de Riemann de genus zero, isto é, na esfera de Riemann. 


\subsubsection{A álgebra de Lie 4-ponto $\mathscr{A}$}

Seguindo Bremner em [Bre95], este primeiro exemplo trata do caso em que o número de polos distintos na esfera de Riemann é exatamente quatro. O trabalho dele foi inspirado em [KN87] de Krichever e Novikov e trouxe ferramentas muito úteis no estudo de álgebras de Lie $n$-ponto. Neste primeiro caso, seremos um pouco mais cuidadosos nos procedimentos e definições e nos casos seguintes seremos um pouco mais sucintos já que os procedimentos são similares e muitas definições não serão mais necessárias.

Considere $\mathfrak{g}$ uma álgebra de Lie complexa de dimensão finita e simples.

\section{$\mathrm{O}$ anel 4-ponto e a álgebra de loop 4-ponto}

Seja $P=\left\{a_{1}, a_{2}, a_{3}, a_{4}\right\}$ o conjunto formado por quatro pontos distintos na esfera de Riemann. O anel 4-ponto $R$ será o anel das funções racionais na esfera de Riemann com polos permitidos somente no conjunto $P$.

Lembre-se que o grupo geral linear projetivo de uma espaço vetorial $V$ é denotado $P G L(V)$ e definido explicitamente como $P G L(V):=A u t(V) / Z(V)$ onde $Z(V)$ é o subgrupo de todas homotetias não nulas de $V$. Caso $V$ seja um $F$-espaço vetorial $n$-dimensional, a notação que adotamos é $P G L_{n}(F)$. Então, como $P G L_{2}(\mathbb{C})$, também conhecido como o grupo das transformações de Möbius, é 3-transitivo, podemos escolher sem perda de generalidade $a_{1}=\infty, a_{2}=0$ e $a_{3}=1$. Isto acontece porque 3 pontos arbitrários sempre podem ser mapeados para 0,1 ou $\infty$ através da razão cruzada.

Definição 2.5.1 (Anel 4-ponto). Escrevendo $a_{4}=a$, nós definimos o anel 4-ponto como $R_{\mathscr{A}}=\mathbb{C}\left[s, s^{-1},(s-1)^{-1},(s-a)^{-1} \mid a \in \mathbb{C} \backslash\{0,1\}\right]$, o anel das funções racionais na esfera de Riemann com polos permitidos somente em $\left\{a_{1}, a_{2}, a_{3}, a_{4}\right\}=\{\infty, 0,1, a\}$.

Similarmente, o anel das funções racionais na esfera de Riemann com polos permitidos somente em um conjunto de $n$ pontos será dito anel n-ponto.

A primeira proposição de [Bre95] nos mostra uma forma conveniente de descrever $R_{\mathscr{A}}$ com apenas 3 geradores.

Proposição 2.5.2. Considere o anel $S=\mathbb{C}\left[t, t^{-1}, u \mid u^{2}=t^{2}-2 b t+1, b \in \mathbb{C} \backslash\{ \pm 1\}\right]$. Teremos $R_{\mathscr{A}} \cong S$ escolhendo $a$ ou $b$ adequadamente.

Demonstração. [Bre95]

Definição 2.5.3 (Álgebra de loop 4-ponto). Diremos que uma álgebra de Lie $\mathscr{L}(\mathscr{A})$ é uma álgebra de loop 4-ponto se esta for composta de um espaço vetorial $\mathfrak{g} \otimes R_{\mathscr{A}}$, onde $R_{\mathscr{A}}$ é o anel 4-ponto, munido das seguintes relações de comutação

$$
[x \otimes f, y \otimes g]=[x, y] \otimes f g
$$

onde $x, y, \in \mathfrak{g}$ e $f, g \in R_{\mathscr{A}}$.

Em geral, uma álgebra de loop n-ponto será definida de forma similar. No caso $n$ ponto, $R$ será um anel $n$-ponto.

\section{A álgebra de Lie 4-ponto}

Justificados os motivos para estudarmos extensões centrais universais de álgebras de Lie, vamos apresentar a extensão central universal da álgebra de loop 4-ponto. Nosso objetivo 
é descrever completamente a extensão central universal da álgebra de Lie $\mathscr{L}(\mathscr{A})$. Seguindo ainda o trabalho de Bremner, podemos explicitar o colchete de Lie dessa extensão central universal utilizando um resultado obtido por Kassel em 1984.

Teorema 2.5.4. Seja $\mathfrak{g}$ uma álgebra de Lie complexa de dimensão finita e seja $R$ uma $\mathbb{C}$-álgebra comutativa e associativa. A extensão central universal de $\mathfrak{g} \otimes R$ é isomorfa como espaço vetorial à $(\mathfrak{g} \otimes R) \oplus \Omega_{R}^{1} / d R$, onde $\Omega_{R}^{1} / d R$ é o espaço de diferenciais de Kähler de $R$ módulo diferenciais exatas.

Demonstração. [Kas84]

As relações de comutação de $(\mathfrak{g} \otimes R) \oplus \Omega_{R}^{1} / d R$ são

$$
\begin{aligned}
{[x \otimes f, y \otimes g] } & =[x y] \otimes f g+(x, y) \overline{f d g}, \\
{[x \otimes f, \omega] } & =0
\end{aligned}
$$

onde $x, y \in \mathfrak{g}, f, g \in R, \omega \in \Omega_{R}^{1} / d R,(\cdot, \cdot)$ denota a forma de Killing em $\mathfrak{g}$.

Definição 2.5.5 (Álgebra de Lie 4-ponto). Nós chamamos $\mathscr{A}$ de álgebra de Lie 4-ponto, onde

$$
\mathscr{A}:=\left(\mathfrak{g} \otimes R_{\mathscr{A}}\right) \oplus \Omega_{R_{\mathscr{A}}}^{1} / d R_{\mathscr{A}}
$$

Em geral, dada uma álgebra de Lie complexa de dimensão finita $\mathfrak{g}$ e $R$ um anel $n$-ponto, vamos denominar por álgebra de Lie n-ponto a álgebra dada por $(\mathfrak{g} \otimes R) \oplus \Omega_{R}^{1} / d R$.

Para tornar explícitas as relações de comutação em $\mathscr{A}$, seguindo [Bre95], primeiro será necessário descrever uma base para $\Omega_{R_{\mathscr{A}}}^{1} / d R_{\mathscr{A}}$.

Antes de tudo, vamos definir os polinômios ultra-esféricos (de Gengenbauer) $P_{k}^{(\lambda)}(x)$.

Definição 2.5.6. [polinômios ultra-esféricos de Gengenbauer] Chamamos polinômios ultraesféricos (de Gengenbauer) os polinômios definidos seguindo o livro de Gabor Szeg, [Sze39] Orthogonal polynomials, pelos valores iniciais $P_{0}^{(\lambda)}(x)=1, P_{1}^{(\lambda)}(x)=2 \lambda x$, onde $\lambda$ é um número racional e pela fórmula recursiva

$$
n P_{n}^{(\lambda)}(x)=2(n+\lambda-1) x P_{n-1}^{(\lambda)}(x)-(n+2 \lambda-2) P_{n-2}^{(\lambda)}(x) .
$$

Em [SW16] encontramos todo o detalhamento desta construção, incluindo a justificativa de que realmente trata-se de um polinômio. A partir desta definição e dado que vamos necessitar apenas do caso em que $\lambda=-1 / 2$, denotaremos $P_{k}(b)=P_{k}^{(-1 / 2)}(b)$ e definiremos

$$
Q_{k}(b)=-\frac{P_{k+2}(b)}{b^{2}-1}
$$

onde $k \in \mathbb{N} \cup\{0\}$.

Será importante conhecer, ao menos, alguns valores de $Q_{k}(b)$. Para referência futura, seguindo [Bre95], os oito primeiros polinômios nessa sequência são

$$
\begin{gathered}
Q_{0}(b)=\frac{1}{2}, Q_{1}(b)=\frac{b}{2}, Q_{2}(b)=\frac{\left(5 b^{2}-1\right)}{8}, Q_{3}(b)=\frac{\left(7 b^{3}-3 b\right)}{8}, \\
Q_{4}(b)=\frac{\left(21 b^{4}-14 b^{2}+1\right)}{16}, Q_{5}(b)=\frac{\left(33 b^{5}-30 b^{3}+5 b\right)}{16}, \\
Q_{6}(b)=\frac{\left(429 b^{6}-495 b^{4}+135 b^{2}-5\right)}{128}, Q_{7}(b)=\frac{\left(715 b^{7}-1001 b^{5}+385 b^{3}-35 b\right)}{128} .
\end{gathered}
$$


Teorema 2.5.7. Uma base para $\Omega_{R_{\mathscr{A}}}^{1} / d R_{\mathscr{A}}$ é $\left\{\overline{t^{-1} d t}, \overline{t^{-2} u d t}, \overline{t^{-1} u d t}\right\}$.

Demonstração. [Bre95]

Teorema 2.5.8. A álgebra de Lie 4 -ponto $\mathscr{A}$ tem uma $\mathbb{Z} / 2 \mathbb{Z}$-graduação em que

$$
\begin{aligned}
& \mathscr{A}^{0}=\mathfrak{g} \otimes \mathbb{C}\left[t, t^{-1}\right] \oplus \mathbb{C} \omega_{0}, \\
& \mathscr{A}^{1}=\mathfrak{g} \otimes \mathbb{C}\left[t, t^{-1}\right] u \oplus \mathbb{C} \omega_{+} \oplus \mathbb{C} \omega_{-},
\end{aligned}
$$

onde $\omega_{0}:=\overline{t^{-1} d t}, \omega_{+}:=\overline{t^{-2} u d t}$, e $\omega_{-}:=\overline{t^{-1} u d t}$. Um subconjunto gerador de $\mathscr{A}$ consiste dos elementos $x \otimes t^{i}$ e $x \otimes t^{j-\frac{1}{2}} u$, onde $x \in \mathfrak{g}, i \in \mathbb{Z}, j \in \mathbb{Z}+\frac{1}{2}$, com elementos centrais $\omega_{0}$, $\omega_{+}$, e $\omega_{-}$. A subálgebra par $\mathscr{A}^{0}$ é uma álgebra com as seguintes relações de comutação:

$$
\left[x \otimes t^{i}, y \otimes t^{j}\right]=[x y] \otimes t^{i+j}+\delta_{i+j, 0}(x, y) j \omega_{0} .
$$

O comutador de dois elementos de $\mathscr{A}^{1}$ resulta em um elemento em $\mathscr{A}^{0}$ :

$$
\begin{aligned}
& {\left[x \otimes t^{i-\frac{1}{2}} u, y \otimes t^{j-\frac{1}{2}} u\right]} \\
& =[x y] \otimes\left(t^{i+j-1}-2 b t^{i+j}+t^{i+j+1}\right)+(x, y) \begin{cases}-2 j b \omega_{0} & \text { para } i+j=0 \\
\frac{1}{2}(j-i) \omega_{0} & \text { para }|i+j|=1 \\
0 & \text { para }|i+j| \geq 2 .\end{cases}
\end{aligned}
$$

O subespaço ímpar $\mathscr{A}^{1}$ é um $\mathscr{A}^{0}$-módulo com relações

$$
\begin{aligned}
& {\left[x \otimes t^{i-\frac{1}{2}} u, y \otimes t^{j}\right]} \\
& =[x y] \otimes\left(t^{i+j-\frac{1}{2}} u+(x, y) \begin{cases}j Q_{i+j-\frac{3}{2}}(b)\left(b \omega_{+}+\omega_{-}\right) & \text {para } i+j \geq \frac{3}{2} \\
j \omega_{ \pm} & \text {para } i+j= \pm \frac{1}{2} \\
j Q_{-i-j-\frac{3}{2}}(b)\left(\omega_{+}+b \omega_{-}\right) & \text {para } i+k \leq-\frac{3}{2}\end{cases} \right.
\end{aligned}
$$

Demonstração. [Bre95]

\subsubsection{A álgebra de Lie 3-ponto $\mathscr{B}$}

Seguindo [CJ13], vamos agora apresentar a álgebra de Lie 3-ponto. A estratégia é muito parecida com a do exemplo anterior.

Considere $\mathfrak{g}=\mathfrak{s l}(2, \mathbb{C})$.

\section{$\mathrm{O}$ anel 3-ponto}

Dado $a \in \mathbb{C} \backslash\{0\}$, os anéis 3-ponto se apresentam de, pelo menos, três formas:

$$
\begin{aligned}
S_{\mathscr{B}} & :=\mathbb{C}\left[s, s^{-1},(s-1)^{-1}\right], \\
R_{\mathscr{B}} & :=\mathbb{C}\left[t, t^{-1}, u \mid u^{2}=t^{2}+4 t\right] \mathrm{e} \\
A_{\mathscr{B}} & :=\mathcal{A}_{a}=\mathbb{C}\left[\left(z^{2}-a^{2}\right)^{k}, z\left(z^{2}-a^{2}\right)^{k} \mid k \in \mathbb{Z}\right] .
\end{aligned}
$$

Em [CJ13] é provado que é possível encontrar isomorfismo entre esses três anéis. Para este trabalho utilizamos $R_{\mathscr{B}}$.

\section{A álgebra de Lie 3-ponto}

Teorema 2.5.9. Uma base para $\Omega_{R_{\mathscr{B}}}^{1} / d R_{\mathscr{B}}$ é $\left\{\omega_{0}:=\overline{t^{-1} d t}, \omega_{1}:=\overline{t^{-1} u d t}\right\}$. 
Demonstração. [CJ13]

De forma similar ao exemplo anterior, apresentamos a seguinte definição.

Definição 2.5.10 (Álgebra de Lie 3-ponto $\mathscr{B}$ ). Nós chamamos $\mathscr{B}$ de álgebra de Lie 3-ponto, e definimos

$$
\mathscr{B}:=\left(\mathfrak{g} \otimes R_{\mathscr{B}}\right) \oplus \Omega_{R_{\mathscr{B}}}^{1} / d R_{\mathscr{B}} .
$$

Considere a seguinte expressão com $k, l \in \mathbb{Z}$

$$
\mu_{k, l}:=-k \frac{\left.(-1)^{k+l} 2^{k+l}(2(k+l)-1) ! !\right)}{(k+l+1) !} .
$$

Ela será útil para descrever as relações de comutação em $\mathscr{B}$.

Teorema 2.5.11. A álgebra de Lie 3 -ponto $\mathscr{B}$ é isomorfa à seguinte álgebra dada por geradores e relações. Considere os geradores $e_{n}, e_{n}^{1}, f_{n}, f_{n}^{1}, h_{n}, h_{n}^{1}, n \in \mathbb{Z}, w_{0}, w_{1}$ e relações dadas por

$$
\begin{aligned}
& {\left[x_{m}, x_{n}\right] }:=\left[x_{m}, x_{n}^{1}\right]=\left[x_{m}^{1}, x_{n}^{1}\right]=0, \text { para } x=e, f \\
& {\left[h_{m}, h_{n}\right] }:=-2 m \delta_{m,-n} \omega_{0}=(n-m) \delta_{m,-n} \omega_{0}, \\
& {\left[h_{m}^{1}, h_{n}^{1}\right] }:=2\left((n+1) \delta_{m+n,-2}+\left(4 n+2 \delta_{m,-n} \omega_{0}\right)=(n-m)\left(\delta_{m+n,-2}+4 \delta_{m+n,-1}\right) \omega_{0},\right. \\
& {\left[h_{m}, h_{n}^{1}\right] }:=-2 \mu_{m, n} \omega_{1} \\
& {\left[\omega_{i}, x_{m}\right] }:=\left[\omega_{i}, \omega_{k}\right]=0 \text { para } i, j \in\{0,1\} \\
& {\left[e_{m}, f_{n}\right]:=h_{m+n}-m \delta_{m,-n} \omega_{0}, } \\
& {\left[e_{m}, f_{n}^{1}\right]:=h_{m+n}^{1}-\mu_{m, n} \omega_{1}=:\left[e_{m}^{1}, f_{n}\right], } \\
& {\left[e_{m}^{1}, f_{n}^{1}\right]:=h_{m+n+2}+4 h_{m+n+1}+\left((n+1) \delta_{m+n,-2}+(4 n+2) \delta_{m+n,-1}\right) \omega_{0} } \\
& {\left[h_{m}, e_{n}\right]:=2 e_{m+n}, } \\
& {\left[h_{m}, e_{n}^{1}\right]:=2 e_{m+n}^{1}=:\left[h_{m}^{1}, e_{m}\right], } \\
& {\left[h_{m}^{1}, e_{n}^{1}\right]:=2 e_{m+n+2}+8 e_{m+n+1}, } \\
& {\left[h_{m}, f_{n}\right]:=-2 f_{m+n}, } \\
& {\left[h_{m}, f_{n}^{1}\right]:=-2 f_{m+n}^{1}=:\left[h_{m}^{1}, f_{m}\right], } \\
& {\left[h_{m}^{1}, f_{n}^{1}\right]:=-2 f_{m+n+2}-8 f_{m+n+1}, }
\end{aligned}
$$

para todo $m, n \in \mathbb{Z}$.

Demonstração. [CJ13]

\subsubsection{A álgebra de Lie elíptica $\mathscr{C}$}

Seguindo [BCF09], vamos agora apresentar a álgebra de Lie elíptica.

Considere $\mathfrak{g}=\mathfrak{s l}(2, \mathbb{C})$. 


\section{$\mathrm{O}$ anel das funções elípticas e a álgebra de loop elíptica}

Adotaremos como definição de anel de funções elípticas o anel $R_{\mathscr{C}}:=\mathbb{C}\left[t, t^{-1}, u \mid u^{2}=\right.$ $\left.t^{3}-2 b t^{2}+t\right]$. A partir disso, podemos definir a álgebra de loop elíptica.

Definição 2.5.12 (álgebra de loop elíptica $\mathscr{L}(\mathscr{C})$ ). Diremos que uma álgebra de Lie $\mathscr{L}(\mathscr{C})$ é uma álgebra de loop elíptica se esta for composta de um espaço vetorial $\mathfrak{g} \otimes R_{\mathscr{C}}$ munido das seguintes relações de comutação

$$
[x \otimes f, y \otimes g]=[x, y] \otimes f g
$$

onde $x, y, \in \mathfrak{g}$ e $f, g \in R_{\mathscr{b}}$.

\section{A álgebra de Lie elíptica}

Definição 2.5.13 (Álgebra de Lie elíptica $\mathscr{C}$ ). Nós chamamos $\mathscr{C}$ de álgebra de Lie elíptica, e definimos

$$
\mathscr{C}:=\left(\mathfrak{g} \otimes R_{\mathscr{C}}\right) \oplus \Omega_{R_{\mathscr{C}}}^{1} / d R_{\mathscr{C}} .
$$

Para tornar explícitas as relações de comutação em $\mathscr{C}$, vamos definir os polinômios de Pollaczeck $\operatorname{Poll}_{k}(b)=\operatorname{Poll}_{k}^{\lambda}(b ; \alpha, \beta, \gamma), \alpha, \beta, \gamma \in \mathbb{C}$ que são dados por recursão

$$
(k+\gamma) \operatorname{Poll}_{k}(b)=2[(k+\lambda+\alpha+\gamma-1) b+\beta] \operatorname{Poll}_{k-1}(b)-(k+2 \lambda+\gamma-2) \operatorname{Poll}_{k-2}(b) .(2 .
$$

Proposição 2.5.14. Considere a sequência de polinômios $p_{k}(b)$, e $q_{k}(b)$, definida por

$$
\overline{t^{k-2} u d t}=p_{k}(b) \overline{t^{-1} u d t}+q_{k}(b) \overline{t^{-2} u d t} .
$$

Esses polinômios são do tipo Pollaczek nos parâmetros $\lambda=-1 / 2, \alpha=0, \beta=-1, \gamma=1 / 2$ com as condições iniciais

$$
p_{0}(b)=0, p_{1}(b)=1, q_{0}(b)=1, q_{1}(b)=0 .
$$

Demonstração. [BCF09]

Teorema 2.5.15. A álgebra de Lie elíptica $\mathscr{C}$ tem uma $\mathbb{Z} / 2 \mathbb{Z}$-graduação onde

$$
\mathscr{C}^{0}=\mathfrak{g} \otimes \mathbb{C}\left[t, t^{-1}\right] \oplus \mathbb{C} \omega_{0}, \mathscr{C}^{1}=\mathfrak{g} \otimes \mathbb{C}\left[t, t^{-1}\right] u \oplus \mathbb{C} \omega_{-} \oplus \mathbb{C} \omega_{+},
$$

$\operatorname{com} \omega_{0}=\overline{t^{-1} d t}, \omega_{-}=\overline{t^{-2} u d t}, \omega_{+}=\overline{t^{-1} u d t}$.

Para quaisquer $x, y \in \mathfrak{g}$, as relações de comutação que definem $\mathscr{C}$ são

$$
\begin{aligned}
{\left[x \otimes t^{i}, y \otimes t^{j}\right]=} & {[x y] \otimes t^{i+j}+\delta_{i+j, 0} j \omega_{0}, } \\
{\left[x \otimes t^{i-1} u, y \otimes t^{j-1} u\right]=} & {[x y] \otimes\left(t^{i+j-1}-2 b t^{i+j}+t^{i+j+1}\right) } \\
& +(x, y) \omega_{0}\left(-2 j b \delta_{i+j, 0}+\frac{1}{2}(j-i)\left(\delta_{i+j,-1}+\delta_{i+j, 1}\right)\right), \\
{\left[x \otimes t^{i-1} u, y \otimes t^{j}\right]=} & {[x y] \otimes t^{i+j-1} u+(x, y) j\left(p_{|i+j|}(b) \omega_{+}+q_{|i+j|}(b) \omega_{-}\right), }
\end{aligned}
$$

$\operatorname{com} i, j, \in \mathbb{Z}$ e $\omega_{0}, \omega_{ \pm}$elementos centrais.

Demonstração. [BCF09] 


\subsubsection{A álgebra de Lie DJKM $\mathscr{D}$}

Seguindo [CFM14], apresentaremos a álgebra de Lie de Date-Jimbo-Kashiwara-Miwa, ou simplesmente DJKM. Considere $\mathfrak{g}$ uma álgebra de Lie complexa de dimensão finita e simples.

Aqui estamos interessados na extensão central universal da álgebra de Lie $\mathfrak{g} \otimes R_{\mathscr{D}}$, onde $R_{\mathscr{D}}=\mathbb{C}\left[t, t^{-1}, u \mid u^{2}=\left(t^{2}-b^{2}\right)\left(t^{2}-c^{2}\right)\right]$ com $b \neq \pm c$ constantes complexas.

\section{A álgebra de Lie DJKM}

Definição 2.5.16 (Álgebra de Lie DJKM $\mathscr{D}$ ). Nós chamamos $\mathscr{D}$ de álgebra de DJKM e definimos

$$
\mathscr{D}:=\left(\mathfrak{g} \otimes R_{\mathscr{D}}\right) \oplus \Omega_{R_{\mathscr{D}}}^{1} / d R_{\mathscr{D}}
$$

Agora necessitaremos dos polinômios $P_{k}:=P_{k}(c)$ dados por recursividade por

$$
(6+2 k) P_{k}(c)=4 k c P_{k-2}(c)-2(k-3) P_{k-4}(c)
$$

para $k \geq 0$. Por meio dos polinômios de Gengenbauer podemos definir os polinômios do tipo $P_{m, n}$ que são descritos em [CFM14]. Se $\omega_{0}=\overline{t^{-1} d t}$ e $\omega_{-k}=\overline{t^{-k} u d t}, k=1,2,3,4$, temos o seguinte teorema que descreve as relações de comutação nesta álgebra.

Teorema 2.5.17. Seja $(\cdot, \cdot)$ a forma de Killing em $\mathfrak{g}$ e defina $\psi_{i j}(c) \in \Omega_{R_{\mathscr{D}}}^{1}$ como

$$
\psi_{i j}(c)= \begin{cases}\omega_{i+j-2} & \text { para } i+j=1,0,-1,-2, \\ P_{-3, i+j-2}(c)\left(\omega_{-3}+c \omega_{-1}\right) & \text { para } i+j=2 n-1 \geq 3, n \in \mathbb{Z}, \\ P_{-3, i+j-2}(c)\left(c \omega_{-3}+\omega_{-1}\right) & \text { para } i+j=-2+1 \leq-3, n \in \mathbb{Z}, \\ \left.P_{-4,|i+j|-2}(c) \omega_{-4}+P_{|i+j|-2}(c) \omega_{-2}\right) & \text { para }|i+j|=2 n \geq 2, n \in \mathbb{Z} .\end{cases}
$$

A álgebra de Lie $\mathscr{D}$ tem uma $\mathbb{Z} / 2 \mathbb{Z}$-graduação

$$
\mathscr{D}=\mathscr{D}^{0} \oplus \mathscr{D}^{1}
$$

onde

$$
\begin{aligned}
& \mathscr{D}^{0}=\left(\mathfrak{g} \otimes \mathbb{C}\left[t, t^{-1}\right]\right) \oplus \mathbb{C} \omega_{0}, \\
& \mathscr{D}^{1}=\left(\mathfrak{g} \otimes \mathbb{C}\left[t, t^{-1}\right] u\right) \oplus \mathbb{C} \omega_{-4} \oplus \mathbb{C} \omega_{-3} \oplus \mathbb{C} \omega_{-2} \oplus \mathbb{C} \omega_{-1} .
\end{aligned}
$$

O colchete de Lie é dado por

$$
\begin{aligned}
{\left[x \otimes t^{i}, y \otimes t^{j}\right]=} & {[x, y] t^{i+j}+\delta_{i+j, 0} j(x, y) \omega_{0} } \\
{\left[x \otimes t^{i-1} u, y \otimes t^{j-1} u\right]=} & {[x, y] \otimes\left(t^{i+j+2}-2 c t^{i+j}+t^{i+j-2}\right) } \\
& +\left(\delta_{i+j,-2}(j+1)+2 c j \delta_{i+j, 0}+(j-1) \delta_{i+j, 2}\right)(x, y) \omega_{0}, \\
{\left[x \otimes t^{i-1} u, y \otimes t^{j}\right]=} & {[x, y] u \otimes t^{i+j-1}+j(x, y) \psi_{i j}(c) . }
\end{aligned}
$$

Demonstração. [CFM14]

Caso $\mathfrak{g}=\mathfrak{s l}(2, \mathbb{C})$ temos um resultado similar ao caso 3-ponto.

Teorema 2.5.18. A álgebra de Lie DJKM $\mathscr{D}$ é isomorfa à seguinte álgebra dada por geradores e relações. Considere os geradores $e_{n}, e_{n}^{1}, f_{n}, f_{n}^{1}, h_{n}, h_{n}^{1}, n \in \mathbb{Z}, w_{0}, w_{-1}, w_{-2}, w_{-3}, w_{-4}$ e 
relações dadas por

$$
\begin{aligned}
{\left[x_{m}, x_{n}\right]:=\left[x_{m}, x_{n}^{1}\right]=\left[x_{m}^{1}, x_{n}^{1}\right]=0, \text { para } x=e, f } \\
{\left[h_{m}, h_{n}\right]:=-2 m \delta_{m,-n} \omega_{0}=(n-m) \delta_{m,-n} \omega_{0}, } \\
{\left[h_{m}^{1}, h_{n}^{1}\right]:=2\left((n+2) \delta_{m+n,-4}-2 c(n+1) \delta_{m+n,-2}+n \delta_{m+n, 0}\right) \omega_{0} } \\
{\left[h_{m}, h_{n}^{1}\right]:=-2 m \psi_{m, n}(c) } \\
{\left[\omega_{i}, x_{m}\right]:=\left[\omega_{i}, \omega_{k}\right]=0 \text { para } x=e, f, h \text { e } i, j \in\{0,1\} } \\
{\left[e_{m}, f_{n}\right]:=h_{m+n}-m \delta_{m,-n} \omega_{0}, } \\
{\left[e_{m}, f_{n}^{1}\right]:=h_{m+n}^{1}-m \psi_{m n}(c)=:\left[e_{m}^{1}, f_{n}\right] } \\
{\left[e_{m}^{1}, f_{n}^{1}\right]:=h_{m+n+4}-2 c h_{m+n+2}+h_{m+n} } \\
{\left[h_{m}, e_{n}\right]:=2 e_{m+n}, } \\
{\left[h_{m}, e_{n}^{1}\right]:=2 e_{m+n}^{1}=:\left[h_{m}^{1}, e_{m}\right] } \\
{\left[h_{m}^{1}, e_{n}^{1}\right]:=2 e_{m+n+4}-4 c e_{m+n+2}+2 e_{m+n}, } \\
{\left[h_{m}, f_{n}\right]:=-2 f_{m+n}, } \\
{\left[h_{m}, f_{n}^{1}\right]:=-2 f_{m+n}^{1}=:\left[h_{m}^{1}, f_{m}\right] } \\
{\left[h_{m}^{1}, f_{n}^{1}\right]:=-2 f_{m+n+4}+4 c f_{m+n+2}-2 f_{m+n}, }
\end{aligned}
$$

para todo $m, n \in \mathbb{Z}$.

Demonstração. [CFM14] 


\section{Capítulo 3}

\section{Módulos de Verma para álgebras de Krichever-Novikov}

A importância de estudar módulos irredutíveis para subálgebras de Heisenberg de uma álgebra de Lie se dá pela possibilidade de induzir módulos irredutíveis para a álgebra de Lie inteira. Como já foi mencionado no Capítulo 0, iremos adaptar a estratégia de [BBFK13] para subálgebras de Heisenberg de algumas álgebras de Krichever-Novikov e exibir com detalhes etapa por etapa, especialmente no primeiro caso onde trabalharemos com uma subálgebra de Heisenberg da álgebra de Lie 4-ponto, definida no Exemplo 2.5.

Apresentamos a seguir alguns resultados antes desconhecidos na classificação de módulos de Verma e $\varphi$-Verma de algumas álgebras de Krichever-Novikov. O método de demonstração para alcançar tais critérios de irredutibilidade será muito similar entre os casos estudados aqui, o que nos faz acreditar na possibilidade de generalizar estes critérios em um trabalho futuro.

Conforme mencionado no Capítulo 0, os resultados novos apresentados nesta seção são:

1. Teorema 3.2.3: Seja $\mathscr{A}$ a álgebra de Lie 4-ponto dada segundo a Definição 2.5.5 e sua subálgebra de Heisenberg $\hat{\mathscr{A}}$ dada segundo a Definição 3.1.1. Considere o módulo $\varphi$-Verma $M_{\hat{\mathscr{A}}, \varphi}$ de $\hat{\mathscr{A}}$ conforme definido em 3.17. $M_{\hat{\mathscr{A}}, \varphi}$ é irredutível se, e somente se, nas relações $(3.16), \kappa_{0} \neq 0$ e $\chi_{+}=0$.

2. Teorema 3.4.3: Seja $\mathscr{B}$ a álgebra de Lie 3-ponto dada segundo a Definição 2.5.10 e sua subálgebra de Heisenberg $\hat{\mathscr{B}}$ dada segundo a Definição 3.3.1. Considere o módulo $\varphi$-Verma $M_{\hat{\mathscr{B}}, \varphi}$ de $\hat{\mathscr{B}}$ conforme definido em 3.40. $M_{\hat{\mathscr{B}}}, \varphi$ é irredutível se, e somente se, nas relações $(3.39), \kappa_{0} \neq 0$.

3. Teorema 3.6.3: Seja $\mathscr{C}$ a álgebra de Lie ica dada segundo a Definição 2.5.12 e sua subálgebra de Heisenberg $\hat{\mathscr{C}}$ dada segundo a Definição 3.5.1. Considere o módulo $\varphi$-Verma $M_{\hat{\mathscr{C}}, \varphi}$ de $\hat{\mathscr{C}}$ conforme definido em 3.69. $M_{\hat{\mathscr{C}}, \varphi}$ é irredutível se, e somente se, nas relações $(3.66), \kappa_{0} \neq 0$.

4. Teorema 3.8.3: Seja $\mathscr{D}$ a álgebra de Lie Date-Jimbo-Kashiwara-Miwa dada segundo a Definição 2.5.16 e sua subálgebra de Heisenberg $\hat{\mathscr{D}}$ dada segundo a Definição 3.7.1. Considere o módulo $\varphi$-Verma $M_{\hat{\mathscr{D}}, \varphi}$ de $\hat{\mathscr{D}}, \varphi$ conforme definido em 3.94. $M_{\hat{\mathscr{D}}, \varphi}$ é irredutível se, e somente se, nas relações $(3.93), \kappa_{0} \neq 0$. 
Obtidos, respectivamente, como generalizações dos Teoremas 3.1.4, 3.3.4, 3.5.4 e 3.7.4, também alcançados neste trabalho.

Apresentaremos a seguir os novos resultados alcançados durante este trabalho. Considere, a partir deste ponto, que $\mathfrak{g}=\mathfrak{s l}(2, \mathbb{C})$.

\subsection{Módulos de Verma para $\hat{\mathscr{A}}$}

Se fizermos o tensor da subálgebra de Cartan $\mathfrak{h}$ de $\mathfrak{g}$ com $R_{\mathscr{A}}$ obteremos uma subálgebra de Heisenberg da álgebra 4-ponto, que pode ser definida por meio de geradores e relações. A definição a seguir foi dada em [Cox08].

Definição 3.1.1. Considerando os geradores $b_{m}, b_{m}^{1}, m \in \mathbb{Z}, 1_{0}, 1_{ \pm}$, que podemos entender por $b_{m}=h \otimes t^{m}, b_{m}^{1}=h \otimes t^{m} u$ com $h \in \mathfrak{h}$ e $1_{0}, 1_{ \pm}$os elementos centrais que geram $\Omega_{R_{\mathscr{A}}}^{1} / d R_{\mathscr{A}}$, com as relações dadas por

$$
\begin{aligned}
{\left[b_{m}, b_{n}\right]=} & 2 n \delta_{m+n, 0} 1_{0} \\
{\left[b_{m}^{1}, b_{n}^{1}\right]=} & (n-m)\left(\delta_{m+n, 0}-2 b \delta_{m+n,-1}+\delta_{m+n,-2}\right) 1_{0} \\
{\left[b_{m}^{1}, b_{n}\right]=} & 2 n\left(Q_{m+n-1}(b)\left(b 1_{+}+1_{-}\right) \delta_{m+n \geq 1}+1_{+} \delta_{m+n, 0}\right. \\
& \left.+1_{-} \delta_{m+n,-1}+Q_{-m-n-2}(b)\left(b 1_{-}+1_{+}\right) \delta_{m+n \leq-2}\right) \\
{\left[b_{m}, 1_{0}\right]=} & {\left[b_{m}^{1}, 1_{0}\right]=\left[b_{m}, 1_{ \pm}\right]=\left[b_{m}^{1}, 1_{ \pm}\right]=\left[1_{0}, 1_{ \pm}\right]=\left[1_{ \pm}, 1_{\mp}\right]=0 }
\end{aligned}
$$

resultam numa subálgebra de Heisenberg de $\mathscr{A}$, denotada por $\hat{\mathscr{A}}$, e que chamaremos de álgebra de Heisenberg 4-ponto.

Definamos os subespaços de $\hat{\mathscr{A}}$

$$
\hat{\mathscr{A}}^{ \pm}=\sum_{n \gtrless 0}\left(\mathbb{C} b_{n}+\mathbb{C} b_{n}^{1}\right) \text { e } \hat{\mathscr{A}}^{0}=\mathbb{C} 1_{-} \oplus \mathbb{C} 1_{0} \oplus \mathbb{C} 1_{+} \oplus \mathbb{C} b_{0} \oplus \mathbb{C} b_{0}^{1} .
$$

Agora observe a seguinte decomposição triangular de $\hat{\mathscr{A}}$

$$
\hat{\mathscr{A}}=\hat{\mathscr{A}}^{-} \oplus \hat{\mathscr{A}}^{0} \oplus \hat{\mathscr{A}}^{+} \text {. }
$$

Neste ponto, considere a álgebra de Borel $\mathfrak{b}_{\hat{\mathscr{A}}}=\hat{\mathscr{A}}^{0} \oplus \hat{\mathscr{A}}^{+}$. Podemos verificar por meio dos colchetes que, de fato, trata-se de uma álgebra.

Seja $\mathbb{C} v$ um $\mathfrak{b}_{\hat{A}^{-}}$módulo tal que $\hat{\mathscr{A}}^{+} \cdot v=0$ e para fixados $\lambda, \mu, \chi_{ \pm}, \kappa_{0} \in \mathbb{C}$

$$
\begin{array}{ll}
b_{0} \cdot v=\lambda v \quad, \quad 1_{ \pm} \cdot v=\chi_{ \pm} v \\
b_{0}^{1} \cdot v=\mu v \quad, \quad 1_{0} \cdot v=\kappa_{0} v
\end{array}
$$

Proposição 3.1.2. No $\mathfrak{b}_{\hat{\mathscr{A}}}$-módulo definido acima, temos

$$
b \chi_{-}+\chi_{+}=0
$$

Demonstração. Observe que conforme já foi dito seção de Exemplos em 2.5,

$$
Q_{0}(b)=\frac{1}{2}
$$


Então,

$$
0=b_{0}^{1} b_{1} v-b_{1} b_{0}^{1} v=\left[b_{0}^{1}, b_{1}\right] v=2\left(Q_{0}(b)\left(b 1_{+}+1_{-}\right)\right) v=\left(b 1_{+}+1_{-}\right) v=\left(b \chi_{+}+\chi_{-}\right) v=0 .
$$

Portanto, $\left(b \chi_{+}+\chi_{-}\right)=0$.

Agora estamos prontos para induzir o módulo de Verma de $\hat{\mathscr{A}}$

$$
M_{\hat{\mathscr{A}}}:=\mathfrak{U}(\hat{\mathscr{A}}) \otimes_{\mathfrak{U}\left(\mathfrak{b}_{\hat{\mathscr{A}}}\right)} \mathbb{C} v .
$$

Seja $w \in M_{\hat{\mathscr{A}}}:=\mathfrak{U}(\hat{\mathscr{A}}) \otimes_{\mathfrak{U}\left(\mathfrak{b}_{\hat{\mathscr{A}}}\right)} \mathbb{C} v$. Pelo Teorema PBW (1.3.1), podemos descrever $M_{\hat{\mathscr{A}}}$ como um $\mathfrak{U}\left(\hat{\mathscr{A}}^{-}\right)$módulo livre. Uma base de $\mathfrak{U}\left(\hat{\mathscr{A}}^{-}\right)$é composta de monômios do tipo $\left(X_{1}^{\alpha_{1}} \cdot X_{2}^{\alpha_{2}} \cdots X_{n}^{\alpha_{n}}\right)$ onde cada $X_{i}$ é um elemento do tipo $b_{i}$ ou $b_{i}^{1}$. Se decidirmos por reordenar algum destes monômios, teremos que considerar que

$$
\begin{aligned}
X_{1}^{\alpha_{1}} \cdot X_{2}^{\alpha_{2}} \cdots X_{i} \cdot X_{j} \cdots X_{n}^{\alpha_{n}} & =X_{1}^{\alpha_{1}} \cdot X_{2}^{\alpha_{2}} \cdots X_{j} \cdot X_{i} \cdots X_{n}^{\alpha_{n}} \\
& +X_{1}^{\alpha_{1}} \cdot X_{2}^{\alpha_{2}} \cdots\left[X_{i}, X_{j}\right] \cdots X_{n}^{\alpha_{n}} .
\end{aligned}
$$

Como para todo $i, j \in \mathbb{Z}_{<0},\left[b_{i}, b_{j}\right],\left[b_{i}^{1}, b_{j}^{1}\right]$ e $\left[b_{i}, b_{j}^{1}\right]$ são elementos centrais em $\hat{\mathscr{A}}$,

$$
\begin{aligned}
X_{1}^{\alpha_{1}} \cdot X_{2}^{\alpha_{2}} \cdots X_{i} \cdot X_{j} \cdots X_{n}^{\alpha_{n}} & =X_{1}^{\alpha_{1}} \cdot X_{2}^{\alpha_{2}} \cdots X_{j} \cdot X_{i} \cdots X_{n}^{\alpha_{n}} \\
& +X_{1}^{\alpha_{1}} \cdot X_{2}^{\alpha_{2}} \cdots \hat{X}_{i} \cdot \hat{X}_{j} \cdots X_{n}^{\alpha_{n}}\left[X_{i}, X_{j}\right]
\end{aligned}
$$

onde $\hat{X}_{k}$ indica a omissão do fator $X_{k}$ no produto.

A partir disso, podemos considerar uma base ordenada de $\mathfrak{U}\left(\hat{\mathscr{A}}^{-}\right)$conveniente. Desta forma, descreveremos um elemento arbitrário $w \in M_{\mathscr{A}}$, como

$$
w=\sum_{(\bar{\alpha}, \bar{\beta})} \xi(\bar{\alpha}, \bar{\beta}) \prod_{i<0} b_{i}^{\alpha_{i}} \prod_{j<0}\left(b_{j}^{1}\right)^{\beta_{j}} v
$$

onde somente um número de $\xi(\bar{\alpha}, \bar{\beta}) \in \mathbb{C}$ é não nulo. Além disso os elementos denotados com uma barra acima serão do tipo $\bar{\gamma}=\left\{\gamma_{-1}, \ldots, \gamma_{N}\right\}$ com $\gamma_{i} \in \mathbb{Z}_{>0}$ para todo $i \in \mathbb{Z}_{<0}$.

Proposição 3.1.3. Se $m \in \mathbb{Z}_{>0}$ e $n \in \mathbb{Z}_{<0}$, teremos que

$$
\begin{aligned}
b_{m}\left(b_{n}\right)^{l} v= & \left(2 \kappa_{0} l n\right) \delta_{m+n, 0}\left(\left(b_{n}\right)^{l-1}\right) v \\
b_{m}\left(b_{n}^{1}\right)^{l} v= & \left(-2 \operatorname{lm} \chi_{+}\right)\left(\delta_{m+n, 0}-b \delta_{m+n,-1}\right. \\
& \left.+Q_{-m-n-2}(b)\left(-b^{2}+1\right) \delta_{m+n, \leq-2}\right)\left(b_{n}^{1}\right)^{l-1} v \\
b_{m}^{1}\left(b_{n}^{1}\right)^{l} v= & \left(\kappa_{0} l(n-m)\right)\left(\delta_{m+n, 0}+2 b \delta_{m+n,-1}+\delta_{m+n,-2}\right)\left(b_{n}^{1}\right)^{l-1} v \\
b_{m}^{1}\left(b_{n}\right)^{l} v= & \left(2 \ln \chi_{+}\right)\left(\delta_{m+n, 0}-b \delta_{m+n,-1}\right. \\
& \left.+Q_{-m-n-2}(b)\left(-b^{2}+1\right) \delta_{m+n, \leq-2}\right)\left(b_{n}^{1}\right)^{l-1} v
\end{aligned}
$$


Demonstração. A prova é dada pelo cálculo direto

$$
\begin{aligned}
b_{m}\left(b_{n}\right)^{l} v= & {\left[b_{m},\left(b_{n}\right)^{l}\right] v+\underline{\left(b_{n}\right)^{l} b_{m} v} } \\
= & l\left(b_{n}\right)^{l-1}\left[b_{m}, b_{n}\right] v \\
= & \left(2 \kappa_{0} l n\right) \delta_{m+n, 0}\left(\left(b_{n}\right)^{l-1}\right) v . \\
b_{m}\left(b_{n}^{1}\right)^{l} v= & {\left[b_{m},\left(b_{n}^{1}\right)^{l}\right] v+\left(b_{n}^{1}\right)^{l} b_{m} v } \\
= & l\left(b_{n}^{1}\right)^{l-1}\left[b_{m}, b_{n}^{1}\right] v \\
= & \left(-2 l m \chi_{+}\right)\left(\delta_{m+n, 0}-b \delta_{m+n,-1}\right. \\
& \left.+Q_{-m-n-2}(b)\left(-b^{2}+1\right) \delta_{m+n, \leq-2}\right)\left(b_{n}^{1}\right)^{l-1} v . \\
b_{m}^{1}\left(b_{n}^{1}\right)^{l} v= & {\left[b_{m}^{1},\left(b_{n}^{1}\right)^{l}\right] v+\left(b_{n}^{1}\right)^{l} b_{m}^{1} v } \\
= & l\left(b_{n}^{1}\right)^{l-1}\left[b_{m}^{1}, b_{n}^{1}\right] v \\
= & \left(\kappa_{0}^{l} l(n-m)\right)\left(\delta_{m+n, 0}+2 b \delta_{m+n,-1}+\delta_{m+n,-2}\right)\left(b_{n}^{1}\right)^{l-1} v . \\
= & {\left[b_{m}^{1},\left(b_{n}\right)^{l}\right] v+\left(b_{n}\right)^{l} b_{m}^{1} v } \\
= & l\left(b_{n}\right)^{l-1}\left[b_{m}^{1}, b_{n}\right] v \\
= & \left(2 \ln \chi_{+}\right)^{1}\left(\delta_{m+n, 0}-b \delta_{m+n,-1}\right. \\
& \left.+Q_{-m-n-2}(b)\left(-b^{2}+1\right) \delta_{m+n, \leq-2}\right)\left(b_{n}^{1}\right)^{l-1} v .
\end{aligned}
$$

Teorema 3.1.4. $M_{\hat{\mathscr{A}}}$ é irredutível se, e somente se, $\kappa_{0} \neq 0$ e $\chi_{+}=0$.

Demonstração. Primeiramente, como trata-se de uma álgebra de Heisenberg, o módulo de Verma $M_{\hat{A}}$ é $\mathbb{Z}$-graduado, de forma que

$$
M_{\hat{\mathscr{A}}}=\bigoplus_{n \in \mathbb{Z}}\left(M_{\hat{\mathscr{A}}}\right)_{n}
$$

onde

$$
\left(M_{\hat{\mathscr{A}}}\right)_{n}=\left\{w \in M_{\hat{\mathscr{A}}}: w=\sum_{(\bar{\alpha}, \bar{\beta})} \xi(\bar{\alpha}, \bar{\beta}) \prod_{i<0} b_{i}^{\alpha_{i}} \prod_{j<0}\left(b_{j}^{1}\right)^{\beta_{j}} v, \sum_{i} \alpha_{i}+\sum_{j} \beta_{j}=n, \forall(\bar{\alpha}, \bar{\beta})\right\} .
$$

Diremos que um elemento $w \in M_{\mathscr{A}}$ tem grau $k$, denotando $\operatorname{deg}(w)=k$, se $w \in\left(M_{\mathscr{A}}\right)_{k}$. A estratégia para demonstração deste teorema será considerar um elemento não nulo $w \in M_{\hat{\mathscr{A}}}$ de grau arbitrário e mostrar que existe $x \in \hat{\mathscr{A}}^{+}$tal que $x w \neq 0$ e $\operatorname{deg}(x w)=0$. Mostrar isso implica que o submódulo gerado por $w$ contém $v$ e consequentemente é todo o $M_{\hat{\mathscr{A}}}$. Como $w$ é um elemento arbitrário não nulo, isso resulta que $M_{\mathscr{A}}$ é irredutível. De forma a alcançar tal demonstração, procederemos por indução em $\operatorname{deg}(w)$.

1. Base de indução:

Suponha que $\operatorname{deg}(w)=1$, então

$$
w=\left(\sum_{i<0} \xi_{i} b_{i}+\sum_{i<0} \xi_{i}^{1} b_{i}^{1}\right) v
$$

onde somente um número de $\xi_{i}, \xi_{j}^{1} \in \mathbb{C}$ é diferente de zero. 
(a) Caso $\xi_{i}=0, \forall i \in \mathbb{Z}_{<0}$, então existe $\xi_{k}^{1} \neq 0$ para algum $k \in \mathbb{Z}_{<0}$. Seja $x \in \hat{\mathscr{A}}^{+}$tal que $x=b_{-k}^{1}$ e $k$ é o menor índice tal que $\xi_{k}^{1} \neq 0$. Teremos que

$$
x w=x \sum_{i<0} \xi_{i}^{1} b_{i}^{1} v=2 \kappa_{0} k \xi_{k}^{1} v .
$$

(b) Caso exista $\xi_{k} \neq 0$ para $k \in \mathbb{Z}_{<0}$. Seja $x \in \hat{\mathscr{A}}^{+}$tal que $x=b_{-k}$. Teremos que

$$
x w=x \sum_{i<0} \xi_{i} b_{i} v=2 \kappa_{0} k \xi_{k} v .
$$

Portanto, em ambos os casos existe $x \in \hat{\mathscr{A}}^{+}$tal que $x w \neq 0$ e $\operatorname{deg}(x w)=0$.

2. Passo de indução: Suponha que para todo $w \in M_{\hat{\mathscr{A}}}$ com grau $n$, exista $y \in \hat{\mathscr{A}}^{+}$tal que $y w \neq 0$ e $\operatorname{deg}(y w)=0$. Agora, $\operatorname{suponha}$ que $\operatorname{deg}(w)=n+1$, então

$$
w=\left(\sum_{i<0} \xi_{i} b_{i}^{(n+1)}+\sum_{i<0} \xi_{i}^{1}\left(b_{i}^{1}\right)^{(n+1)}+\sum_{(\bar{\alpha}, \bar{\beta})} \xi(\bar{\alpha}, \bar{\beta}) \prod_{i<0} b_{i}^{\alpha_{i}} \prod_{j<0}\left(b_{j}^{1}\right)^{\beta_{j}}\right) v
$$

onde somente um número de $\xi_{i}, \xi_{i}^{1}$ e $\xi(\bar{\alpha}, \bar{\beta})$ é diferente de zero.

(a) Caso exista $\xi(\bar{\alpha}, \bar{\beta}) \neq 0$, tome $\xi(\bar{k}, \bar{l}) \neq 0$ e sejam $m$ o maior índice tal que $k_{m} \neq 0$ e $x \in \hat{A}^{+}$tal que $x=b_{-m}$. Além disso, reordene os monômios de maneira que:

i. caso $\prod_{i<0} b_{i}^{\alpha_{i}} \prod_{j<0}\left(b_{j}^{1}\right)^{\beta_{j}}$ possua como fator $b_{m}$, reescreva-o como $b_{m}^{\alpha_{m}} \prod_{i<0, i \neq m}$ $b_{i}^{\alpha_{i}} \prod_{j<0}\left(b_{j}^{1}\right)^{\beta_{j}}$ considerando as novas constantes e os novos monômios que surgirão desta mudança, como observado em (3.7).

ii. caso contrário, reescreva-o como $b_{i_{0}}^{\alpha_{i_{0}}} \prod_{i<i_{0}} b_{i}^{\alpha_{i}} \prod_{j<0}\left(b_{j}^{1}\right)^{\beta_{j}}, i_{0} \neq m$ é o primeiro fator à esquerda.

Então, com as constantes apropriadas,

$$
\begin{aligned}
w= & \left(\sum_{i<0} \xi_{i} b_{i}^{(n+1)}+\sum_{i<0} \xi_{i}^{1}\left(b_{i}^{1}\right)^{(n+1)}+\sum_{(\bar{\alpha}, \bar{\beta})} \xi(\bar{\alpha}, \bar{\beta}) \prod_{i<0} b_{i}^{\alpha_{i}} \prod_{j<0}\left(b_{j}^{1}\right)^{\beta_{j}}\right) v \\
= & \left(\sum_{i<0} \tilde{\xi}_{i} b_{i}^{(n+1)}+\sum_{i<0} \tilde{\xi}_{i}^{1}\left(b_{i}^{1}\right)^{(n+1)}+\sum_{(\bar{\alpha}, \bar{\beta})} \tilde{\xi}(\bar{\alpha}, \bar{\beta}) b_{m}^{\alpha_{m}} \prod_{i \neq m} b_{i}^{\alpha_{i}} \prod_{j<0}\left(b_{j}^{1}\right)^{\beta_{j}}\right. \\
& \left.+\sum_{(\bar{\alpha}, \bar{\beta})} \tilde{\xi}(\bar{\alpha}, \bar{\beta}) b_{i_{0}}^{\alpha_{i_{0}}} \prod_{i \neq m} b_{i}^{\alpha_{i}} \prod_{j<0}\left(b_{j}^{1}\right)^{\beta_{j}}\right) v .
\end{aligned}
$$


Aplicando $x$ em $w$ obtemos

$$
\begin{aligned}
x w= & \tilde{\xi}_{m} 2 \kappa_{0}(n+1) m b_{m}^{n} v+0+\sum_{(\bar{\alpha}, \bar{\beta})} \tilde{\xi}(\bar{\alpha}, \bar{\beta}) b_{-m} b_{m}^{\alpha_{m}} \prod_{i \neq m} b_{i}^{\alpha_{i}} \prod_{j<0}\left(b_{j}^{1}\right)^{\beta_{j}} v \\
& +\sum_{(\bar{\alpha}, \bar{\beta})} \tilde{\xi}(\bar{\alpha}, \bar{\beta}) b_{-m} b_{i_{0}}^{\alpha_{i_{0}}} \prod_{i \neq m} b_{i}^{\alpha_{i}} \prod_{j<0}\left(b_{j}^{1}\right)^{\beta_{j}} v \\
= & \tilde{\xi}_{m} 2 \kappa_{0}(n+1) m b_{m}^{n} v \\
& +\sum_{(\bar{\alpha}, \bar{\beta})} \tilde{\xi}(\bar{\alpha}, \bar{\beta}) \alpha_{m}\left(2 m \kappa_{0}\right) b_{m}^{\alpha_{m}-1} \prod_{i \neq m} b_{i}^{\alpha_{i}} \prod_{j<0}\left(b_{j}^{1}\right)^{\beta_{j}} v .
\end{aligned}
$$

Portanto, $x w \neq 0$ e $\operatorname{deg}(x w)=n$. Pela hipótese de indução conseguimos reduzir $x w$ ao grau 0.

(b) Caso $\xi(\bar{\alpha}, \bar{\beta})=0, \forall(\bar{\alpha}, \bar{\beta})$.

i. Se existe $\xi_{k} \neq 0$ tome $x=b_{-k}$ e então $x w=2 \kappa_{0}(n+1) k \xi_{k}^{n} b_{k}^{n} v$.

ii. Se $\xi_{i}=0$ para todo $i \in \mathbb{Z}_{<0}$, tome $x=b_{-k}^{1}$, onde $k$ é o menor índice tal que $\xi_{k}^{1} \neq 0$. Teremos que $x w=2 \kappa_{0}(n+1) k\left(b_{k}^{1}\right) v$.

Portanto, $x w \neq 0$ e $\operatorname{deg}(x w)=n$.

Finalmente podemos afirmar que, por indução, dado um elemento arbitrário $w \in M_{\hat{\mathscr{A}}}$ há um elemento $x \in \hat{\mathscr{A}}^{+}$tal que $x w \neq 0$ e $\operatorname{deg}(x w)=0$, isto é, que gera todo o módulo $M_{\hat{\mathscr{A}}}$. Portanto, $M_{\hat{\mathscr{A}}}$ não possui submódulos próprios; em outras palavras, é irredutível como queríamos demonstrar.

Suponha que $\kappa_{0}=\chi_{+}=0$. Pela Proposição 3.1.2, $\chi_{-}=0$. Considere o subconjunto $N_{\hat{\mathscr{A}}}$ de $M_{\hat{\mathscr{A}}}$ dado por

$$
N_{\hat{\mathscr{A}}}=\bigoplus_{n \in \mathbb{Z} \backslash\{0\}}\left(M_{\hat{\mathscr{A}}}\right)_{n} .
$$

(i) De fato o subconjunto $N_{\hat{\mathscr{A}}}$ é um submódulo de $M_{\hat{\mathscr{A}}}$. Dados $x, y \in N_{\hat{\mathscr{A}}}$ e $a, b \in \mathfrak{U}(\hat{\mathscr{A}})$, $(x a+y b) \in N_{\hat{\mathscr{B}}}$. Por consequência da Proposição 3.1.3 junta à hipótese de que $\kappa_{0}=0$, se $x \in \mathfrak{U}\left(\hat{\mathscr{A}}^{+}\right)$então $x \cdot N_{\hat{\mathscr{A}}}=0$, enquanto que se $y \in \mathfrak{U}\left(\hat{\mathscr{A}}^{-}\right)$e $b \in N_{\hat{\mathscr{A}}}$, então $\operatorname{deg}(y b) \geq \operatorname{deg}(b)$.

(ii) Observe que $\sum_{i \in \mathbb{Z}} \xi_{i} v \notin N_{\hat{B}}$, onde $\xi_{i} \in \mathbb{C}$ para todo $i \in \mathbb{Z}$.

Por (i) e (ii) $N_{\hat{\mathscr{A}}}$ é um submódulo próprio de $M_{\hat{\mathscr{A}}}$.

\subsection{Módulos $\varphi$-Verma para $\hat{\mathscr{A}}$}

Podemos agora introduzir os módulos $\varphi$-Verma $\hat{\mathscr{A}}$, conforme mostramos a construção na Seção 1.7.1.

Como os geradores de $\hat{\mathscr{A}}$ são indexados em $\mathbb{Z}$, estenderemos o domínio da função $\varphi$. Considere daqui em diante a seguinte função $\varphi$ :

$$
\varphi: \mathbb{Z} \backslash\{0\} \rightarrow\{ \pm\}
$$

com a condição adicional de que $\varphi(n)=+\Leftrightarrow \varphi(-n)=-$. 
Definamos os subespaços de $\hat{\mathscr{A}}$

$$
\hat{\mathscr{A}}_{\varphi}^{ \pm}=\left(\sum_{\substack{n \in \mathbb{Z}<0 \\ \varphi(n)=\mp}}\left(\mathbb{C} b_{n}+\mathbb{C} b_{n}^{1}\right)\right) \oplus\left(\sum_{\substack{n \in \mathbb{Z}>0 \\ \varphi(n)= \pm}}\left(\mathbb{C} b_{n}+\mathbb{C} b_{n}^{1}\right)\right) .
$$

Para um número $n \in \mathbb{Z} \backslash\{0\}$ denotemos por $\operatorname{sgn}(n)$ a função que indica o sinal de $n$, isto é, $\operatorname{sgn}(n)=+\Leftrightarrow n>0$ e $\operatorname{sgn}(n)=-\Leftrightarrow n<0$. Observe que, por exemplo, os geradores de $\hat{\mathscr{A}}_{\varphi}^{+}$são $b_{m}, b_{n}^{1}$ tais que $\varphi(m)=\operatorname{sgn}(m)$ e $\varphi(n)=\operatorname{sgn}(n)$. Utilizando-nos deste raciocínio, podemos descrever os subespaços $\hat{\mathscr{A}}_{\varphi}^{ \pm}$de forma abreviada como

$$
\hat{\mathscr{A}}_{\varphi}^{+}=\left(\sum_{\substack{n \in \mathbb{Z} \backslash\{0\} \\ \varphi(n)=\operatorname{sgn}(n)}}\left(\mathbb{C} b_{n}+\mathbb{C} b_{n}^{1}\right)\right) \text { e } \hat{\mathscr{A}}_{\varphi}^{-}=\left(\sum_{\substack{n \in \mathbb{Z} \backslash\{0\} \\ \varphi(n) \neq \operatorname{sgn}(n)}}\left(\mathbb{C} b_{n}+\mathbb{C} b_{n}^{1}\right)\right) .
$$

Observe a seguinte decomposição de $\hat{\mathscr{A}}$

$$
\hat{\mathscr{A}}=\hat{\mathscr{A}}_{\varphi}^{-} \oplus \hat{\mathscr{A}}^{0} \oplus \hat{\mathscr{A}}_{\varphi}^{+} .
$$

Considere a álgebra tipo de Borel $\mathfrak{b}_{\hat{\mathscr{\varphi}}_{\varphi}}=\hat{\mathscr{A}}^{0} \oplus \hat{\mathscr{A}}_{\varphi}^{+}$. Podemos verificar por meio dos colchetes que, de fato, trata-se de uma álgebra.

Seja $\mathbb{C} v$ um $\mathfrak{b}_{\mathscr{A}_{\varphi}}$-módulo tal que $\hat{\mathscr{A}}_{\varphi}^{+} \cdot v=0$ e para fixados $\lambda, \mu, \chi_{ \pm}, \kappa_{0} \in \mathbb{C}$

$$
\begin{array}{ll}
b_{0} \cdot v=\lambda v \quad, \quad 1_{ \pm} \cdot v=\chi_{ \pm} v \\
b_{1}^{1} \cdot v=\mu v \quad, \quad 1_{0} \cdot v=\kappa_{0} v
\end{array}
$$

Proposição 3.2.1. No $\mathfrak{b}_{\hat{A}_{\varphi}}$-módulo definido acima, temos

$$
b \chi_{-}+\chi_{+}=0
$$

Demonstração. Veja a demonstração da Proposição 3.1.2.

Agora estamos prontos para introduzir o módulo $\varphi$-Verma de $\hat{\mathscr{A}}$

$$
M_{\hat{\mathscr{A}}, \varphi}=\mathfrak{U}(\hat{\mathscr{A}}) \otimes_{\mathfrak{U}\left(\mathfrak{b}_{\hat{A}_{\varphi}}\right)} \mathbb{C} v
$$

Podemos considerar uma base ordenada de $\mathfrak{U}\left(\hat{\mathscr{A}}_{\varphi}^{-}\right)$conveniente. Desta forma, descrevemos um elemento arbitrário $w \in M_{\hat{\mathscr{A}}, \varphi}$, como

$$
w=\left(\sum_{(\bar{\alpha}, \bar{\beta})} \xi(\bar{\alpha}, \bar{\beta}) \prod_{\substack{i \in \mathbb{Z} \backslash\{0\} \\ \varphi(i) \neq \operatorname{sgn}(i)}} b_{i}^{\alpha_{i}} \prod_{\substack{j \in \mathbb{Z} \backslash\{0\} \\ \varphi(j) \neq \operatorname{sgn}(j)}}\left(b_{j}^{1}\right)^{\beta_{j}}\right) v
$$

onde somente um número de $\xi(\bar{\alpha}, \bar{\beta}) \in \mathbb{C}$ é não nulo. Além disso os elementos denotados com uma barra acima serão do tipo $\bar{\gamma}=\left\{\gamma_{N_{1}}, \ldots, \gamma_{N_{k}}\right\}$ com $\gamma_{i} \in \mathbb{Z}_{>0}$ para todo $N_{i} \in \mathbb{Z} \backslash\{0\}$. 
Proposição 3.2.2. Se $m, n \in \mathbb{Z} \backslash\{0\}, \varphi(m)=\operatorname{sgn}(m)$ e $\varphi(n) \neq \operatorname{sgn}(n)$, então

$$
\begin{aligned}
b_{m}\left(b_{n}\right)^{l} v= & \left(2 \kappa_{0} \ln \right) \delta_{m+n, 0}\left(\left(b_{n}\right)^{l-1}\right) v \\
b_{m}\left(b_{n}^{1}\right)^{l} v= & \left(-2 \operatorname{lm} \chi_{+}\right)\left(\delta_{m+n, 0}-b \delta_{m+n,-1}\right. \\
& \left.+Q_{-m-n-2}(b)\left(-b^{2}+1\right) \delta_{m+n, \leq-2}\right)\left(b_{n}^{1}\right)^{l-1} v \\
b_{m}^{1}\left(b_{n}^{1}\right)^{l} v= & \left(\kappa_{0} l(n-m)\right)\left(\delta_{m+n, 0}+2 b \delta_{m+n,-1}+\delta_{m+n,-2}\right)\left(b_{n}^{1}\right)^{l-1} v \\
b_{m}^{1}\left(b_{n}\right)^{l} v= & \left(2 \ln \chi_{+}\right)\left(\delta_{m+n, 0}-b \delta_{m+n,-1}\right. \\
& \left.+Q_{-m-n-2}(b)\left(-b^{2}+1\right) \delta_{m+n, \leq-2}\right)\left(b_{n}^{1}\right)^{l-1} v
\end{aligned}
$$

Demonstração. A prova é dada pelo cálculo direto

$$
\begin{aligned}
b_{m}\left(b_{n}\right)^{l} v= & {\left[b_{m},\left(b_{n}\right)^{l}\right] v+\left(b_{n}\right)^{l} b_{m} v } \\
= & l\left(b_{n}\right)^{l-1}\left[b_{m}, b_{n}\right] v \\
= & \left(2 \kappa_{0} l n\right) \delta_{m+n, 0}\left(\left(b_{n}\right)^{l-1}\right) v . \\
b_{m}\left(b_{n}^{1}\right)^{l} v= & {\left[b_{m},\left(b_{n}^{1}\right)^{l}\right] v+\left(b_{n}^{1}\right)^{l} b_{m} v } \\
= & l\left(b_{n}^{1}\right)^{l-1}\left[b_{m}, b_{n}^{1}\right] v \\
= & \left(-2 l m \chi_{+}\right)\left(\delta_{m+n, 0}-b \delta_{m+n,-1}\right. \\
& \left.+Q_{-m-n-2}(b)\left(-b^{2}+1\right) \delta_{m+n, \leq-2}\right)\left(b_{n}^{1}\right)^{l-1} v . \\
b_{m}^{1}\left(b_{n}^{1}\right)^{l} v= & {\left[b_{m}^{1},\left(b_{n}^{1}\right)^{l}\right] v+\left(b_{n}^{1}\right)^{l} b_{m}^{1} v } \\
= & l\left(b_{n}^{1}\right)^{l-1}\left[b_{m}^{1}, b_{n}^{1}\right] v \\
= & \left(\kappa_{0} l(n-m)\right)\left(\delta_{m+n, 0}+2 b \delta_{m+n,-1}+\delta_{m+n,-2}\right)\left(b_{n}^{1}\right)^{l-1} v . \\
= & {\left[b_{m}^{1},\left(b_{n}\right)^{l}\right] v+\left(b_{n}\right)^{l} b_{m}^{1} v } \\
= & l\left(b_{n}\right)^{l-1}\left[b_{m}^{1}, b_{n}\right] v \\
= & \left(2 \ln \chi_{+}\right)\left(\delta_{m+n, 0}-b \delta_{m+n,-1}\right. \\
& \left.+Q_{-m-n-2}(b)\left(-b^{2}+1\right) \delta_{m+n, \leq-2}\right)\left(b_{n}^{1}\right)^{l-1} v .
\end{aligned}
$$

Como podemos observar, a demonstração é similar àquela da Proposição 3.1.3.

Teorema 3.2.3. O módulo $\varphi$-Verma $M_{\hat{\mathscr{A}}, \varphi}$ é irredutível se, e somente se, $\kappa_{0} \neq 0$ e $\chi_{+}=0$. Demonstração. Como trata-se de uma álgebra de Heisenberg, o módulo $\varphi$-Verma $M_{\hat{\mathscr{A}}, \varphi}$ é $\mathbb{Z}$-graduado, de forma que

$$
M_{\hat{\mathscr{A}}, \varphi}=\bigoplus_{n \in \mathbb{Z}}\left(M_{\hat{\mathscr{A}}, \varphi}\right)_{n}
$$

onde

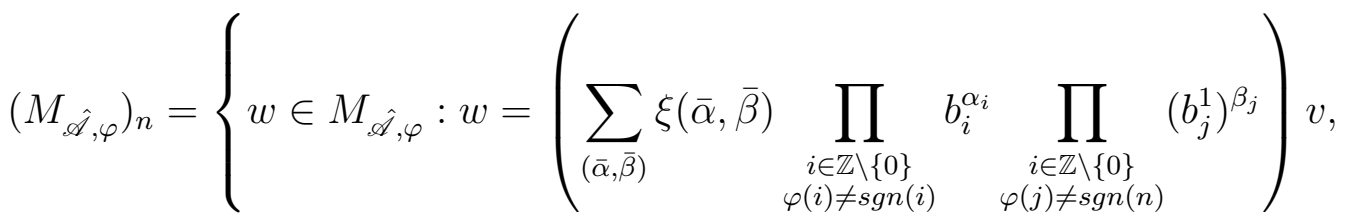

$$
\begin{aligned}
& \left.\sum_{i} \alpha_{i}+\sum_{j} \beta_{j}=n, \forall(\bar{\alpha}, \bar{\beta})\right\} \text {. }
\end{aligned}
$$

Faremos indução em $\operatorname{deg}(w)$ para exibir que todo elemento de $M_{\hat{\mathscr{A}}, \varphi}$ pode gerar o próprio módulo $M_{\hat{\mathscr{A}}, \varphi}$. 
1. Base de indução:

Suponha que $\operatorname{deg}(w)=1$, então

onde somente um número de $\xi_{i}, \xi_{j}^{1} \in \mathbb{C}$ é diferente de zero.

(a) Caso $\xi_{i}=0, \forall i \in \mathbb{Z} \backslash\{0\}$, então existe $\xi_{k}^{1} \neq 0$ para algum $k \in \mathbb{Z} \backslash\{0\}$. Seja $x \in \hat{\mathscr{A}}_{\varphi}^{+}$tal que $x=b_{-k}^{1}$ e $k$ é o menor índice tal que $\xi_{k}^{1} \neq 0$. Teremos que

$$
x w=x \sum_{\substack{i \in \mathbb{Z} \backslash\{0\} \\ \varphi(i) \neq s g n(i)}} \xi_{i}^{1} b_{i}^{1} v=2 \kappa_{0} k \xi_{k}^{1} v .
$$

(b) Caso exista $\xi_{k} \neq 0$ para $k \in \mathbb{Z} \backslash\{0\}$. Seja $x \in \hat{\mathscr{A}}_{\varphi}^{+}$tal que $x=b_{-k}$. Teremos que

$$
x w=x \sum_{\substack{i \in \mathbb{Z} \backslash\{0\} \\ \varphi(i) \neq \operatorname{sgn}(i)}} \xi_{i} b_{i} v=2 \kappa_{0} k \xi_{k} v .
$$

Portanto, em ambos os casos existe $x \in \hat{\mathscr{A}}_{\varphi}^{+}$tal que $x w \neq 0$ e $\operatorname{deg}(x w)=0$.

2. Passo de indução: Suponha que para todo $w \in M_{\hat{\mathscr{A}}_{, \varphi}}$ com grau $n$, exista $y \in \hat{\mathscr{A}}_{\varphi}^{+}$tal que $y w \neq 0$ e $\operatorname{deg}(y w)=0$. Agora, $\operatorname{suponha}$ que $\operatorname{deg}(w)=n+1$, então

$$
w=\left(\sum_{\substack{i \in \mathbb{Z} \backslash\{0\} \\ \varphi(i) \neq \operatorname{sgn}(i)}} \xi_{i} b_{i}^{(n+1)}+\sum_{\substack{i \in \mathbb{Z} \backslash\{0\} \\ \varphi(i) \neq \operatorname{sgn}(i)}} \xi_{i}^{1}\left(b_{i}^{1}\right)^{(n+1)}+\sum_{(\bar{\alpha}, \bar{\beta})} \xi(\bar{\alpha}, \bar{\beta}) \prod_{\substack{i \in \mathbb{Z} \backslash\{0\} \\ \varphi(i) \neq \operatorname{sgn}(i)}} b_{i}^{\alpha_{i}} \prod_{\substack{j \in \mathbb{Z} \backslash\{0\} \\ \varphi(j) \neq \operatorname{sgn}(j)}}\left(b_{j}^{1}\right)^{\beta_{j}}\right) v
$$

onde somente um número de $\xi_{i}, \xi_{i}^{1}$ e $\xi(\bar{\alpha}, \bar{\beta})$ é diferente de zero.

(a) Caso exista $\xi(\bar{\alpha}, \bar{\beta}) \neq 0$, então tome $\xi(\bar{k}, \bar{l}) \neq 0$ e sejam $m$ o maior índice tal que $k_{m} \neq 0$ e $x \in \hat{\mathscr{A}}_{\varphi}^{+}$tal que $x=b_{-m}$. Além disso, reordene os monômios de maneira que:

i. caso $\prod_{i \in \mathbb{Z} \backslash\{0\}} b_{i}^{\alpha_{i}} \prod_{j \in \mathbb{Z} \backslash\{0\}}\left(b_{j}^{1}\right)^{\beta_{j}}$ possua como fator $b_{m}$, reescreva-o como $\varphi(i) \neq \operatorname{sgn}(i) \quad{ }_{\varphi(j) \neq \operatorname{sgn}(j)}$ $b_{m}^{\alpha_{m}} \prod_{\substack{i \in \mathbb{Z} \backslash\{0, m\} \\ \varphi(i) \neq \operatorname{sgn}(i)}} b_{i}^{\alpha_{i}} \prod_{\substack{j \in \mathbb{Z} \backslash\{0\} \\ \varphi(j) \neq \operatorname{sgn}(j)}}\left(b_{j}^{1}\right)^{\beta_{j}}$ considerando as novas constantes e os novos monômios que surgirão desta mudança, como observado em (3.7).

ii. caso contrário, reescreva-o como $b_{i_{0}}^{\alpha_{i_{0}}} \prod_{\substack{i \in \mathbb{Z} \backslash\{0\} \\ \varphi(i) \neq \operatorname{sgn}(i)}} b_{i}^{\alpha_{i}} \prod_{\substack{j \in \mathbb{Z} \backslash\{0\} \\ \varphi(j) \neq \operatorname{sgn}(j)}}\left(b_{j}^{1}\right)^{\beta_{j}}, i_{0} \neq m$ é o primeiro fator à esquerda. 
Então, com as constantes apropriadas,

$$
\begin{aligned}
& w=\left(\sum_{\substack{i \in \mathbb{Z} \backslash\{0\} \\
\varphi(i) \neq \operatorname{sgn}(i)}} \xi_{i} b_{i}^{(n+1)}+\sum_{\substack{i \in \mathbb{Z} \backslash\{0\} \\
\varphi(i) \neq \operatorname{sgn}(i)}} \xi_{i}^{1}\left(b_{i}^{1}\right)^{(n+1)}\right. \\
& \left.+\sum_{(\bar{\alpha}, \bar{\beta})} \xi(\bar{\alpha}, \bar{\beta}) \prod_{\substack{i \in \mathbb{Z} \backslash\{0\} \\
\varphi(i) \neq \operatorname{sgn}(i)}} b_{i}^{\alpha_{i}} \prod_{\substack{j \in \mathbb{Z} \backslash\{0\} \\
\varphi(j) \neq \operatorname{sgn}(j)}}\left(b_{j}^{1}\right)^{\beta_{j}}\right) v \\
& =\left(\sum_{\substack{i \in \mathbb{Z} \backslash\{0\} \\
\varphi(i) \neq \operatorname{sgn}(i)}} \tilde{\xi}_{i} b_{i}^{(n+1)}+\sum_{\substack{i \in \mathbb{Z} \backslash\{0\} \\
\varphi(i) \neq \operatorname{sgn}(i)}} \tilde{\xi}_{i}^{1}\left(b_{i}^{1}\right)^{(n+1)}\right. \\
& +\sum_{(\bar{\alpha}, \bar{\beta})} \tilde{\xi}(\bar{\alpha}, \bar{\beta}) b_{m}^{\alpha_{m}} \prod_{\substack{i \in \mathbb{Z} \backslash\{0, m\} \\
\varphi(i) \neq s g n(i)}} b_{i}^{\alpha_{i}} \prod_{\substack{j \in \mathbb{Z} \backslash\{0\} \\
\varphi(j) \neq \operatorname{sgn}(j)}}\left(b_{j}^{1}\right)^{\beta_{j}} \\
& \left.+\sum_{(\bar{\alpha}, \bar{\beta})} \tilde{\xi}(\bar{\alpha}, \bar{\beta}) b_{i_{0}}^{\alpha_{i_{0}}} \prod_{\substack{i \in \mathbb{Z} \backslash\{0, m\} \\
\varphi(i) \neq \operatorname{sgn}(i)}} b_{i}^{\alpha_{i}} \prod_{\substack{j \in \mathbb{Z} \backslash\{0\} \\
\varphi(j) \neq \operatorname{sgn}(j)}}\left(b_{j}^{1}\right)^{\beta_{j}}\right) v .
\end{aligned}
$$

Aplicando $x$ em $w$ obtemos

$$
\begin{aligned}
x w= & \tilde{\xi}_{m} 2 \kappa_{0}(n+1) m b_{m}^{n} v+0+\sum_{(\bar{\alpha}, \bar{\beta})} \tilde{\xi}(\bar{\alpha}, \bar{\beta}) b_{-m} b_{m}^{\alpha_{m}} \prod_{\substack{i \in \mathbb{Z} \backslash\{0, m\} \\
\varphi(i) \neq s g n(i)}} b_{i}^{\alpha_{i}} \prod_{\substack{j \in \mathbb{Z} \backslash\{0\} \\
\varphi(j) \neq s g n(j)}}\left(b_{j}^{1}\right)^{\beta_{j}} v \\
& +\sum_{(\bar{\alpha}, \bar{\beta})} \tilde{\xi}(\bar{\alpha}, \bar{\beta}) b_{-m} b_{i_{0}}^{\alpha_{i_{0}}} \prod_{\substack{i \in \mathbb{Z} \backslash\{0, m\} \\
\varphi(i) \neq s g n(i)}} b_{i}^{\alpha_{i}} \prod_{\substack{j \in \mathbb{Z} \backslash\{0\} \\
\varphi(j) \neq s g n(j)}}\left(b_{j}^{1}\right)^{\beta_{j}} v \\
= & \tilde{\xi}_{m} 2 \kappa_{0}(n+1) m b_{m}^{n} v \\
& +\sum_{(\bar{\alpha}, \bar{\beta})} \tilde{\xi}(\bar{\alpha}, \bar{\beta}) \alpha_{m}\left(2 m \kappa_{0}\right) b_{m}^{\alpha_{m}-1} \prod_{\substack{i \in \mathbb{Z} \backslash\{0, m\} \\
\varphi(i) \neq s g n(i)}} b_{i}^{\alpha_{i}} \prod_{\substack{j \in \mathbb{Z} \backslash\{0\} \\
\varphi(j) \neq s g n(j)}}\left(b_{j}^{1}\right)^{\beta_{j}} v .
\end{aligned}
$$

Portanto, $x w \neq 0$ e $\operatorname{deg}(x w)=n$. Pela hipótese de indução conseguimos reduzir $x w$ ao grau 0.

(b) Caso $\xi(\bar{\alpha}, \bar{\beta})=0, \forall(\bar{\alpha}, \bar{\beta})$.

i. Se existe $\xi_{k} \neq 0$ tome $x=b_{-k}$ e então $x w=2 \kappa_{0}(n+1) k \xi_{k}^{n} b_{k}^{n} v$.

ii. Se $\xi_{i}=0$ para todo $i \in \mathbb{Z}$, tome $x=b_{-k}^{1}$, onde $k$ é o menor índice tal que $\xi_{k}^{1} \neq 0$. Teremos que $x w=2 \kappa_{0}(n+1) k\left(b_{k}^{1}\right) v$.

Portanto, $x w \neq 0$ e $\operatorname{deg}(x w)=n$.

Portanto, $M_{\hat{\mathscr{A}}, \varphi}$ não possui submódulos próprios; em outras palavras, é irredutível como queríamos demonstrar.

Suponha que $\kappa_{0}=\chi_{+}=0$. Pela Proposição 3.2.1, $\chi_{-}=0$. Considere o subconjunto $N_{\hat{A}, \varphi}$ 
de $M_{\hat{\mathscr{A}}, \varphi}$ dado por

$$
N_{\hat{\mathscr{A}}, \varphi}=\bigoplus_{n \in \mathbb{Z} \backslash\{0\}}\left(M_{\hat{\mathscr{A}}}\right)_{n} .
$$

(i) De fato o subconjunto $N_{\hat{\mathscr{A}}, \varphi}$ é um submódulo de $M_{\hat{\mathscr{A}}, \varphi}$. Dados $x, y \in N_{\hat{\mathscr{A}}, \varphi}$ e $a, b \in$ $\mathfrak{U}(\hat{\mathscr{A}}),(x a+y b) \in N_{\hat{\mathscr{A}}, \varphi}$. Por consequência da Proposição 3.2 .2 junta à hipótese de que $\kappa_{0}=0$, se $x \in \mathfrak{U}\left(\hat{\mathscr{A}}_{\varphi}^{+}\right)$então $x \cdot N_{\hat{\mathscr{A}}_{, \varphi}}=0$, enquanto que se $y \in \mathfrak{U}\left(\hat{\mathscr{A}}_{\varphi}^{-}\right)$e $b \in N_{\hat{\mathscr{A}}, \varphi}$, então $\operatorname{deg}(y b) \geq \operatorname{deg}(b)$.

(ii) Observe que $\sum_{i \in \mathbb{Z} \backslash\{0\}} \xi_{i} v \notin N_{\hat{\mathscr{A}}}$, onde $\xi_{i} \in \mathbb{C}$ para todo $i \in \mathbb{Z} \backslash\{0\}$.

Por (i) e (ii) $N_{\hat{\mathscr{A}}, \varphi}$ é um submódulo próprio de $M_{\hat{\mathscr{A}}, \varphi}$.

\subsection{Módulos de Verma para $\hat{\mathscr{B}}$}

De forma parecida com o caso anterior, se fizermos o tensor da subálgebra de Cartan $\mathfrak{h}$ de $\mathfrak{g}$ com $R_{\mathscr{B}}$ obteremos uma subálgebra de Heisenberg da álgebra 3-ponto, que pode ser definida por meio de geradores e relações. A definição a seguir foi dada em [CJ13].

Definição 3.3.1. Considerando os geradores $b_{m}, b_{m}^{1}, m \in \mathbb{Z}, 1_{0}, 1_{1}$, que podemos entender por $b_{m}=h \otimes t^{m}, b_{m}^{1}=h \otimes t^{m} u$ com $h \in \mathfrak{h}$ e $1_{0}, 1_{ \pm}$os elementos centrais que geram $\Omega_{R_{\mathscr{B}}}^{1} / d R_{\mathscr{B}}$, com as relações dadas por

$$
\begin{aligned}
& {\left[b_{m}, b_{n}\right]=-2 m \delta_{m+n, 0} 1_{0}} \\
& {\left[b_{m}^{1}, b_{n}^{1}\right]=(n-m)\left(\delta_{m+n,-2}+4 \delta_{m+n,-1}\right) 1_{0}} \\
& {\left[b_{m}^{1}, b_{n}\right]=2 n \mu_{n, m} 1_{1}} \\
& {\left[b_{m}, 1_{0}\right]=\left[b_{m}^{1}, 1_{0}\right]=\left[b_{m}, 1_{1}\right]=\left[b_{m}^{1}, 1_{1}\right]=\left[1_{0}, 1_{1}\right]=\left[1_{ \pm}, 1_{1}\right]=0}
\end{aligned}
$$

resultam na subálgebra de Heisenberg de $\mathscr{B}$, denotada por $\hat{\mathscr{B}}$, e que chamaremos de álgebra de Heisenberg 3-ponto.

Definamos os subespaços de $\hat{\mathscr{B}}$

$$
\hat{\mathscr{B}}^{ \pm}=\sum_{n \gtrless 0}\left(\mathbb{C} b_{n}+\mathbb{C} b_{n}^{1}\right) \text { e } \hat{\mathscr{B}}^{0}=\mathbb{C} 1_{-} \oplus \mathbb{C} 1_{0} \oplus \mathbb{C} 1_{+} \oplus \mathbb{C} b_{0} \oplus \mathbb{C} b_{0}^{1} .
$$

Agora observe a seguinte decomposição de $\hat{\mathscr{B}}$

$$
\hat{\mathscr{B}}=\hat{\mathscr{B}}^{-} \oplus \hat{\mathscr{B}}^{0} \oplus \hat{\mathscr{B}}^{+} .
$$

Considere a álgebra tipo de Borel $\mathfrak{b}_{\hat{\mathscr{B}}}=\hat{\mathscr{B}}^{0} \oplus \hat{\mathscr{B}}^{+}$. Podemos verificar por meio dos colchetes que, de fato, trata-se de uma álgebra.

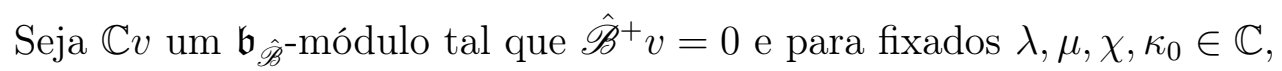

$$
\begin{array}{ll}
b_{0} v=\lambda v \quad, \quad 1_{1} v=\chi v \\
b_{0}^{1} v=\mu v \quad, \quad 1_{0} v=\kappa_{0} v
\end{array}
$$

Proposição 3.3.2. No $\mathfrak{b}_{\hat{\mathscr{B}}}$-módulo definido acima, temos

$$
\chi=0
$$


Demonstração. Observe que podemos calcular utilizando as definições da seção de Exemplos 2.5 que

$$
\mu_{1,1}=-960 \neq 0
$$

Então,

$$
0=b_{1}^{1} b_{1} v-b_{1} b_{1}^{1} v=\left[b_{1}^{1}, b_{1}\right] v=2 \mu_{1,1} 1_{1} v=2 \chi \mu_{1,1} v \Leftrightarrow \chi=0 .
$$

Portanto, $\chi=0$.

Agora estamos prontos para induzir o módulo de Verma de $\hat{\mathscr{B}}$

$$
M_{\hat{\mathscr{B}}}=\mathfrak{U}(\hat{\mathscr{B}}) \otimes_{\mathfrak{U}\left(\mathfrak{b}_{\hat{\mathscr{B}}}\right)} \mathbb{C} v
$$

Seja $w \in M_{\hat{\mathscr{B}}}=\mathfrak{U}(\hat{\mathscr{B}}) \otimes_{\mathfrak{U}\left(\mathfrak{b}_{\hat{\mathscr{B}}}\right)} \mathbb{C} v$. Podemos descrever $w$, como

$$
w=\sum_{(\bar{\alpha}, \bar{\beta})} \xi(\bar{\alpha}, \bar{\beta}) \prod_{i<0} b_{i}^{\alpha_{i}} \prod_{j<0}\left(b_{j}^{1}\right)^{\beta_{j}} v
$$

onde somente um número de $\xi(\bar{\alpha}, \bar{\beta}) \in \mathbb{C}$ é não nulo. Além disso, $\bar{\gamma}=\left\{\gamma_{-1}, \ldots, \gamma_{N}\right\}$ com $\gamma_{i} \in \mathbb{Z}_{>0}$ para todo $i \in \mathbb{Z}_{<0}$.

Proposição 3.3.3. Se $m \in \mathbb{Z}_{>0}$ e $n \in \mathbb{Z}_{<0}$, teremos que

$$
\begin{aligned}
& b_{m}\left(b_{n}\right)^{l} v=\left(-2 l m \kappa_{0}\right) \delta_{m+n, 0}\left(\left(b_{n}\right)^{l-1}\right) v \\
& b_{m}\left(b_{n}^{1}\right)^{l} v=0 \\
& b_{m}^{1}\left(b_{n}^{1}\right)^{l} v=\left(\kappa_{0} l(n-m)\right)\left(\delta_{m+n,-2}+4 \delta_{m+n,-1}\right)\left(b_{n}^{1}\right)^{l-1} v, \\
& b_{m}^{1}\left(b_{n}\right)^{l} v=0 .
\end{aligned}
$$

Demonstração. A prova é dada pelo cálculo direto

$$
\begin{aligned}
b_{m}\left(b_{n}\right)^{l} v & =\left[b_{m},\left(b_{n}\right)^{l}\right] v+\left(b_{n}\right)^{l} b_{m} v \\
& =l\left(b_{n}\right)^{l-1}\left[b_{m}, b_{n}\right] v \\
& =\left(-2 l m \kappa_{0}\right) \delta_{m+n, 0}\left(\left(b_{n}\right)^{l-1}\right) v . \\
b_{m}\left(b_{n}^{1}\right)^{l} v & =\left[b_{m},\left(b_{n}^{1}\right)^{l}\right] v+\left(b_{n}^{1}\right)^{l} b_{m} v \\
& =l\left(b_{n}^{1}\right)^{l-1}\left[b_{m}, b_{n}^{1}\right] v \\
& =0 . \\
b_{m}^{1}\left(b_{n}^{1}\right)^{l} v & =\left[b_{m}^{1},\left(b_{n}^{1}\right)^{l}\right] v+\left(b_{n}^{1}\right)^{l} b_{m}^{1} v \\
& =l\left(b_{n}^{1}\right)^{l-1}\left[b_{m}^{1}, b_{n}^{1}\right] v \\
& =\left(\kappa_{0}^{l} l(n-m)\right)\left(\delta_{m+n,-2}+4 \delta_{m+n,-1}\right)\left(b_{n}^{1}\right)^{l-1} v . \\
b_{m}^{1}\left(b_{n}\right)^{l} & =\left[b_{m}^{1},\left(b_{n}\right)^{l}\right] v+\left(b_{n}\right)^{l} b_{m}^{1} v \\
& =l\left(b_{n}\right)^{l-1}\left[b_{m}^{1}, b_{n}\right] v \\
& =0 .
\end{aligned}
$$

Teorema 3.3.4. $M_{\hat{\mathscr{B}}}$ é irredutível se, e somente se, $\kappa_{0} \neq 0$.

Demonstração. Como trata-se de uma álgebra de Heisenberg, o módulo de Verma $M_{\hat{\mathscr{B}}}$ é 
$\mathbb{Z}$-graduado, de forma que

$$
M_{\hat{\mathscr{B}}}=\bigoplus_{n \in \mathbb{Z}}\left(M_{\hat{\mathscr{B}}}\right)_{n}
$$

onde

$$
\left(M_{\hat{\mathscr{B}}}\right)_{n}=\left\{w \in M_{\hat{\mathscr{B}}}: w=\sum_{(\bar{\alpha}, \bar{\beta})} \xi(\bar{\alpha}, \bar{\beta}) \prod_{i<0} b_{i}^{\alpha_{i}} \prod_{j<0}\left(b_{j}^{1}\right)^{\beta_{j}} v, \sum_{i} \alpha_{i}+\sum_{j} \beta_{j}=n, \forall(\bar{\alpha}, \bar{\beta})\right\} .
$$

Diremos que um elemento $w \in M_{\hat{\mathscr{B}}}$ tem grau $k$, denotando $\operatorname{deg}(w)=k$, se $w \in\left(M_{\hat{\mathscr{B}}}\right)_{k}$.

A estratégia para demonstração deste teorema será similar à do Teorema 3.1.4, isto é, mostrar que assumindo a hipótese de que $\kappa_{0} \neq 0, M_{\mathscr{B}}$ não possui submódulos próprios. Procederemos novamente por indução em $\operatorname{deg}(w)$.

1. Base de indução:

Suponha que $\operatorname{deg}(w)=1$, então

$$
w=\left(\sum_{i<0} \xi_{i} b_{i}+\sum_{i<0} \xi_{i}^{1} b_{i}^{1}\right) v
$$

onde somente um número de $\xi_{i}, \xi_{i}^{1} \in \mathbb{C}$ é diferente de zero.

(a) Caso $\xi_{i}=0, \forall i \in \mathbb{Z}_{<0}$, então existe $\xi_{k}^{1} \neq 0$ para algum $k \in \mathbb{Z}_{<0}$. Seja $x \in \mathscr{B}^{+}$tal que $x=b_{-k}^{1}$ e $k$ é o menor índice tal que $\xi_{k-1}^{1} \neq 0$. Teremos que

$$
x w=x \sum_{i<0} \xi_{i}^{1} b_{i}^{1} v=2(k-1) \kappa_{0} \xi_{k-1}^{1} v .
$$

(b) Caso exista $\xi_{k} \neq 0$ para $k \in \mathbb{Z}_{<0}$. Seja $x \in \mathscr{B}^{+}$tal que $x=b_{-k}$. Teremos que

$$
x w=x \sum_{i<0} \xi_{i} b_{i} v=2 \kappa_{0} k \xi_{k} v .
$$

Portanto, em ambos os casos existe $x \in \mathscr{B}^{+}$tal que $x w \neq 0$ e $\operatorname{deg}(x w)=0$.

2. Passo de indução: Suponha que para todo $w \in M_{\mathscr{B}}$ com grau $n$, exista $y \in \mathscr{B}^{+}$tal que $y w \neq 0$ e $\operatorname{deg}(y w)=0$. Agora, $\operatorname{suponha}$ que $\operatorname{deg}(w)=n+1$, então

$$
w=\left(\sum_{i<0} \xi_{i} b_{i}^{(n+1)}+\sum_{i<0} \xi_{i}^{1}\left(b_{i}^{1}\right)^{(n+1)}+\sum_{(\bar{\alpha}, \bar{\beta})} \xi(\bar{\alpha}, \bar{\beta}) \prod_{i<0} b_{i}^{\alpha_{i}} \prod_{j<0}\left(b_{j}^{1}\right)^{\beta_{j}}\right) v
$$

onde somente um número de $\xi_{i}, \xi_{i}^{1}$ e $\xi(\bar{\alpha}, \bar{\beta})$ é diferente de zero.

(a) Caso exista $\xi(\bar{\alpha}, \bar{\beta}) \neq 0$, então tome $\xi(\bar{k}, \bar{l}) \neq 0$ e sejam $m$ o maior índice tal que $k_{m} \neq 0$ e $x \in \mathscr{B}^{+}$tal que $x=b_{-m}$. Além disso, reordene os monômios de maneira que:

i. caso $\prod_{i<0} b_{i}^{\alpha_{i}} \prod_{j<0}\left(b_{j}^{1}\right)^{\beta_{j}}$ possua como fator $b_{m}$, reescreva-o como $b_{m}^{\alpha_{m}} \prod_{i<0, i \neq m}$ $b_{i}^{\alpha_{i}} \prod_{j<0}\left(b_{j}^{1}\right)^{\beta_{j}}$ considerando as novas constantes e os novos monômios que surgirão desta mudança, como observado em (3.7). 
ii. caso contrário, reescreva-o como $b_{i_{0}}^{\alpha_{i_{0}}} \prod_{i<i_{0}} b_{i}^{\alpha_{i}} \prod_{j<0}\left(b_{j}^{1}\right)^{\beta_{j}}, i_{0} \neq m$ é o primeiro fator à esquerda.

Então, com as constantes apropriadas,

$$
\begin{aligned}
w= & \left(\sum_{i<0} \xi_{i} b_{i}^{(n+1)}+\sum_{i<0} \xi_{i}^{1}\left(b_{i}^{1}\right)^{(n+1)}+\sum_{(\bar{\alpha}, \bar{\beta})} \xi(\bar{\alpha}, \bar{\beta}) \prod_{i<0} b_{i}^{\alpha_{i}} \prod_{j<0}\left(b_{j}^{1}\right)^{\beta_{j}}\right) v \\
= & \left(\sum_{i<0} \tilde{\xi}_{i} b_{i}^{(n+1)}+\sum_{i<0} \tilde{\xi}_{i}^{1}\left(b_{i}^{1}\right)^{(n+1)}+\sum_{(\bar{\alpha}, \bar{\beta})} \tilde{\xi}(\bar{\alpha}, \bar{\beta}) b_{m}^{\alpha_{m}} \prod_{i \neq m} b_{i}^{\alpha_{i}} \prod_{j<0}\left(b_{j}^{1}\right)^{\beta_{j}}\right. \\
& \left.+\sum_{(\bar{\alpha}, \bar{\beta})} \tilde{\xi}(\bar{\alpha}, \bar{\beta}) b_{i_{0}}^{\alpha_{i}} b_{m}^{\alpha_{m}} \prod_{i \neq m} b_{i}^{\alpha_{i}} \prod_{j<0}\left(b_{j}^{1}\right)^{\beta_{j}}\right) v .
\end{aligned}
$$

Aplicando $x$ em $w$ obtemos

$$
\begin{aligned}
x w= & -\tilde{\xi_{m}} 2 \kappa_{0}(n+1) m b_{m}^{n} v+0+\sum_{(\bar{\alpha}, \bar{\beta})} \tilde{\xi}(\bar{\alpha}, \bar{\beta}) b_{-m} b_{m}^{\alpha_{m}} \prod_{i \neq m} b_{i}^{\alpha_{i}} \prod_{j<0}\left(b_{j}^{1}\right)^{\beta_{j}} v \\
& +\sum_{(\bar{\alpha}, \bar{\beta})} \tilde{\xi}(\bar{\alpha}, \bar{\beta}) b_{-m} b_{i_{0}}^{\alpha_{i_{0}}} b_{m}^{\alpha_{m}} \prod_{i \neq m} b_{i}^{\alpha_{i}} \prod_{j<0}\left(b_{j}^{1}\right)^{\beta_{j}} v \\
= & -\tilde{\xi_{m}} 2 \kappa_{0}(n+1) m b_{m}^{n} v \\
& +\sum_{(\bar{\alpha}, \bar{\beta})} \tilde{\xi}(\bar{\alpha}, \bar{\beta}) \alpha_{m}\left(2 m \kappa_{0}\right) b_{m}^{\alpha_{m}-1} \prod_{i \neq m} b_{i}^{\alpha_{i}} \prod_{j<0}\left(b_{j}^{1}\right)^{\beta_{j}} v
\end{aligned}
$$

Portanto, $x w \neq 0$ e $\operatorname{deg}(x w)=n$. Pela hipótese de indução conseguimos reduzir $x w$ ao grau 0.

(b) Caso $\xi(\bar{\alpha}, \bar{\beta})=0, \forall(\bar{\alpha}, \bar{\beta})$.

i. Se existe $\xi_{k} \neq 0$ tome $x=b_{-k}$ e então $x w=2 \kappa_{0}(n+1) k \xi_{k}^{n} b_{k}^{n} v$.

ii. Se $\xi_{i}=0$ para todo $i \in \mathbb{Z}_{<0}$, tome $x=b_{-k}^{1}$, onde $k$ é o menor índice tal que $\xi_{k-1}^{1} \neq 0$. Teremos que $x w=4 \xi_{k-1}^{1} \kappa_{0}(n+1)\left(b_{k-1}^{1}\right) v$.

Portanto, $x w \neq 0$ e $\operatorname{deg}(x w)=n$.

Finalmente podemos afirmar que, por indução, dado um elemento arbitrário $w \in M_{\hat{\mathscr{B}}}$ há um elemento $x \in \mathscr{B}^{+}$tal que $x w \neq 0$ e $\operatorname{deg}(x w)=0$, isto é, que gera todo o módulo $M_{\hat{\mathscr{B}}}$. Portanto, $M_{\hat{\mathscr{B}}}$ não possui submódulos próprios; em outras palavras, é irredutível como queríamos demonstrar.

Suponha que $\kappa_{0}=0$. Considere o subconjunto $N_{\hat{\mathscr{B}}}$ de $M_{\hat{\mathscr{B}}}$ dado por

$$
N_{\hat{\mathscr{B}}}=\bigoplus_{n \in \mathbb{Z} \backslash\{0\}}\left(M_{\hat{\mathscr{B}}}\right)_{n} .
$$

(i) De fato o subconjunto $N_{\hat{\mathscr{B}}}$ é um submódulo de $M_{\hat{\mathscr{B}}}$. Dados $x, y \in N_{\hat{\mathscr{B}}}$ e $a, b \in \mathfrak{U}(\hat{\mathscr{B}})$, $(x a+y b) \in N_{\hat{\mathscr{B}}}$. Por consequência da Proposição 3.3.3 junta à hipótese de que $\kappa_{0}=0$, se $x \in \mathfrak{U}\left(\hat{\mathscr{B}}^{+}\right)$então $x \cdot N_{\hat{\mathscr{B}}}=0$, enquanto que se $y \in \mathfrak{U}\left(\hat{\mathscr{B}}^{-}\right)$e $b \in N_{\hat{\mathscr{B}}}$, então $\operatorname{deg}(y b) \geq \operatorname{deg}(b)$.

(ii) Observe que $\sum_{i \in \mathbb{Z}} \xi_{i} v \notin N_{\hat{\mathscr{B}}}$, onde $\xi_{i} \in \mathbb{C}$ para todo $i \in \mathbb{Z}$. 
Por (i) e (ii) $N_{\hat{\mathscr{B}}}$ é um submódulo próprio de $M_{\hat{\mathscr{B}}}$.

\subsection{Módulos $\varphi$-Verma para $\hat{\mathscr{B}}$}

Definamos os subespaços de $\hat{\mathscr{B}}$

$$
\hat{\mathscr{B}}_{\varphi}^{ \pm}=\left(\sum_{\substack{n \in \mathbb{Z}_{<0} \\ \varphi(n)=\mp}}\left(\mathbb{C} b_{n}+\mathbb{C} b_{n}^{1}\right)\right) \oplus\left(\sum_{\substack{m \in \mathbb{Z}_{>0} \\ \varphi(m)= \pm}}\left(\mathbb{C} b_{m}+\mathbb{C} b_{m}^{1}\right)\right) .
$$

Agora observe a seguinte decomposição de $\hat{\mathscr{B}}$

$$
\hat{\mathscr{B}}=\hat{\mathscr{B}}_{\varphi}^{-} \oplus \hat{\mathscr{B}}^{0} \oplus \hat{\mathscr{B}}_{\varphi}^{+}
$$

Considere a álgebra tipo de Borel $\mathfrak{b}_{\hat{\mathscr{B}} \varphi}=\hat{\mathscr{B}}^{0} \oplus \hat{\mathscr{B}}_{\varphi}^{+}$. Podemos verificar por meio dos colchetes que, de fato, trata-se de uma álgebra.

Seja $\mathbb{C} v$ um $\mathfrak{b}_{\hat{\mathscr{B}} \varphi}$-módulo tal que $\hat{\mathscr{B}}_{\varphi}^{+} \cdot v=0$ e para fixados $\lambda, \mu, \chi, \kappa_{0} \in \mathbb{C}$

$$
\begin{array}{ll}
b_{0} \cdot v=\lambda v \quad, \quad 1_{1} \cdot v=\chi v \\
b_{1}^{1} \cdot v=\mu v \quad, \quad 1_{0} \cdot v=\kappa_{0} v
\end{array}
$$

Proposição 3.4.1. No $\mathfrak{b}_{\hat{\mathscr{B}} \varphi}$-módulo definido acima, temos

$$
\chi=0
$$

Demonstração. $\quad$ (i) Se $\varphi(1)=-$ então $\varphi(-1)=+$, portanto

$$
0=b_{-1}^{1} b_{-1} v-b_{1} b_{-1}^{1} v=\left[b_{-1}^{1}, b_{-1}\right] v=2 \mu_{1,1} 1_{1} v=2 \chi \mu_{-1,-1} v \Leftrightarrow \chi=0 .
$$

(ii) Caso $\varphi(1)=+$, basta repetirmos a demonstração da Proposição 3.3.2.

Agora estamos prontos para introduzir o módulo $\varphi$-Verma de $\hat{\mathscr{B}}$

$$
M_{\hat{\mathscr{B}}, \varphi}=\mathfrak{U}(\hat{\mathscr{B}}) \otimes_{\mathfrak{b}_{\hat{\mathscr{B}} \varphi}} \mathbb{C} v
$$

Podemos descrever um elemento arbitrário $w \in M_{\hat{\mathscr{B}}, \varphi}$, como

$$
w=\sum_{(\bar{\alpha}, \bar{\beta})} \xi(\bar{\alpha}, \bar{\beta}) \prod_{\substack{i \in \mathbb{Z} \\ \varphi(i) \neq \operatorname{sgn}(i)}} b_{i}^{\alpha_{i}} \prod_{\substack{j \in \mathbb{Z} \\ \varphi(j) \neq s g n(j)}}\left(b_{j}^{1}\right)^{\beta_{j}} v
$$

onde somente um número de $\xi(\bar{\alpha}, \bar{\beta}) \in \mathbb{C}$ é não nulo. Além disso, $\bar{\gamma}=\left\{\gamma_{N_{1}}, \ldots, \gamma_{N_{k}}\right\}$ com $\gamma_{i} \in \mathbb{Z}_{>0}$ para todo $i \in \mathbb{Z} \backslash\{0\}$. 
Proposição 3.4.2. Se $m, n \in \mathbb{Z} \backslash\{0\}, \varphi(m)=\operatorname{sgn}(m)$ e $\varphi(n) \neq \operatorname{sgn}(n)$, então

$$
\begin{aligned}
b_{m}\left(b_{n}\right)^{l} v & =\left(-2 l m \kappa_{0}\right) \delta_{m+n, 0}\left(\left(b_{n}\right)^{l-1}\right) v \\
b_{m}\left(b_{n}^{1}\right)^{l} v & =0 \\
b_{m}^{1}\left(b_{n}^{1}\right)^{l} v & =\left(\kappa_{0} l(n-m)\right)\left(\delta_{m+n,-2}+4 \delta_{m+n,-1}\right)\left(b_{n}^{1}\right)^{l-1} v \\
b_{m}^{1}\left(b_{n}\right)^{l} v & =0 .
\end{aligned}
$$

Demonstração. A prova é dada pelo cálculo direto

$$
\begin{array}{rl}
b_{m}\left(b_{n}\right)^{l} v & =\left[b_{m},\left(b_{n}\right)^{l}\right] v+\left(b_{n}\right)^{l} b_{m} v \\
& =l\left(b_{n}\right)^{l-1}\left[b_{m}, b_{n}\right] v \\
& =\left(-2 l m \kappa_{0}\right) \delta_{m+n, 0}\left(\left(b_{n}\right)^{l-1}\right) v . \\
b_{m}\left(b_{n}^{1}\right)^{l} v & =\left[b_{m},\left(b_{n}^{1}\right)^{l}\right] v+\left(b_{n}^{1}\right)^{l} b_{m} v \\
& =l\left(b_{n}^{1}\right)^{l-1}\left[b_{m}, b_{n}^{1}\right] v \\
& =0 . \\
b_{m}^{1}\left(b_{n}^{1}\right)^{l} v & =\left[b_{m}^{1},\left(b_{n}^{1}\right)^{l}\right] v+\left(b_{n}^{1}\right)^{l} b_{m}^{1} v \\
& =l\left(b_{n}^{1}\right)^{l-1}\left[b_{m}^{1}, b_{n}^{1}\right] v \\
& =\left(\kappa_{0}^{l} l(n-m)\right)\left(\delta_{m+n,-2}+4 \delta_{m+n,-1}\right)\left(b_{n}^{1}\right)^{l-1} v . \\
& =\left[b_{m}^{1},\left(b_{n}\right)^{l}\right] v+\left(b_{n}\right)^{l} b_{m}^{1} v \\
b_{m}^{1}\left(b_{n}\right)^{l} v & l\left(b_{n}\right)^{l-1}\left[b_{m}^{1}, b_{n}\right] v \\
& =0 .
\end{array}
$$

Teorema 3.4.3. O módulo $\varphi$-Verma $M_{\hat{\mathscr{B}}, \varphi}$ é irredutível se, e somente se, $\kappa_{0} \neq 0$.

Demonstração. Como trata-se de uma álgebra de Heisenberg, o módulo $\varphi$-Verma $M_{\hat{\mathscr{B}}, \varphi}$ é $\mathbb{Z}$-graduado, de forma que

$$
M_{\hat{\mathscr{B}}, \varphi}=\bigoplus_{n \in \mathbb{Z}}\left(M_{\hat{\mathscr{B}}, \varphi}\right)_{n}
$$

onde

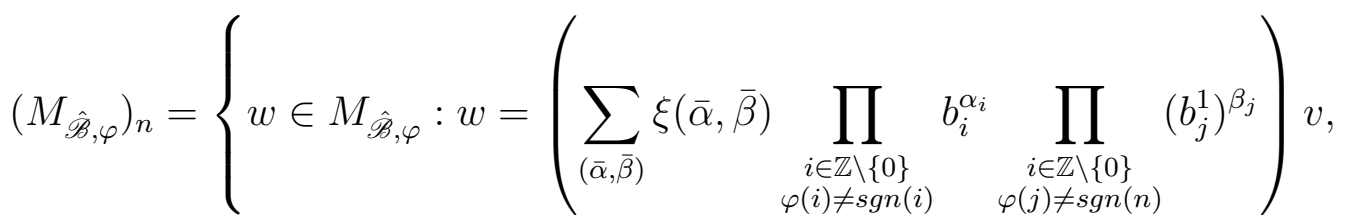

$$
\begin{aligned}
& \left.\sum_{i} \alpha_{i}+\sum_{j} \beta_{j}=n, \forall(\bar{\alpha}, \bar{\beta})\right\} \text {. }
\end{aligned}
$$

Procederemos por indução em $\operatorname{deg}(w)$ para exibir que todo elemento de $M_{\hat{\mathscr{B}}, \varphi}$ pode gerar o próprio módulo $M_{\hat{\mathscr{B}}, \varphi}$.

1. Base de indução: 
Suponha que $\operatorname{deg}(w)=1$, então

$$
w=\left(\sum_{\substack{i \in \mathbb{Z} \\ \varphi(i) \neq \operatorname{sgn}(i)}} \xi_{i} b_{i}+\sum_{\substack{i \in \mathbb{Z} \\ \varphi(i) \neq s g n(i)}} \xi_{i}^{1} b_{i}^{1}\right) v
$$

onde somente um número de $\xi_{i}, \xi_{i}^{1} \in \mathbb{C}$ é diferente de zero.

(a) Caso $\xi_{i}=0, \forall i \in \mathbb{Z}_{<0}$, então existe $\xi_{k}^{1} \neq 0$ para algum $k \in \mathbb{Z}_{<0}$. Seja $x \in \mathscr{B}_{\varphi}^{+}$tal que $x=b_{-k}^{1}$ e $k$ é o menor índice tal que $\xi_{k-1}^{1} \neq 0$. Teremos que

$$
x w=x \sum_{\substack{i \in \mathbb{Z} \\ \varphi(i) \neq s g n(i)}} \xi_{i}^{1} b_{i}^{1} v=2(k-1) \kappa_{0} \xi_{k-1}^{1} v .
$$

(b) Caso exista $\xi_{k} \neq 0$ para $k \in \mathbb{Z} \backslash\{0\}$. Seja $x \in \mathscr{B}_{\varphi}^{+}$tal que $x=b_{-k}$. Teremos que

$$
x w=x \sum_{\substack{i \in \mathbb{Z} \\ \varphi(i) \neq \operatorname{sgn}(i)}} \xi_{i} b_{i} v=2 \kappa_{0} k \xi_{k} v .
$$

Portanto, em ambos os casos existe $\hat{\mathscr{B}}_{\varphi}^{+}$tal que $x w \neq 0$ e $\operatorname{deg}(x w)=0$.

2. Passo de indução: Suponha que para todo $v \in M_{\hat{\mathscr{B}}, \varphi}$ com grau $n$, exista $y \in \mathscr{B}^{+}$tal que $y w \neq 0$ e $\operatorname{deg}(y w)=0$. Agora, $\operatorname{suponha}$ que $\operatorname{deg}(w)=n+1$, então

$$
w=\left(\sum_{\substack{i \in \mathbb{Z} \\ \varphi(i) \neq \operatorname{sgn}(i)}} \xi_{i} b_{i}^{(n+1)}+\sum_{\substack{i \in \mathbb{Z} \\ \varphi(i) \neq s g n(i)}} \xi_{i}^{1}\left(b_{i}^{1}\right)^{(n+1)}+\sum_{(\bar{\alpha}, \bar{\beta})} \xi(\bar{\alpha}, \bar{\beta}) \prod_{\substack{i \in \mathbb{Z} \\ \varphi(i) \neq \operatorname{sgn}(i)}} b_{i}^{\alpha_{i}} \prod_{\substack{j \in \mathbb{Z} \\ \varphi(j) \neq \operatorname{sgn}(j)}}\left(b_{j}^{1}\right)^{\beta_{j}}\right) v
$$

onde somente um número de $\xi_{i}, \xi_{i}^{1}$ e $\xi(\bar{\alpha}, \bar{\beta})$ é diferente de zero.

(a) Caso exista $\xi(\bar{\alpha}, \bar{\beta}) \neq 0$, então tome $\xi(\bar{k}, \bar{l}) \neq 0$ e sejam $m$ o maior índice tal que $k_{m} \neq 0$ e $x \in \mathscr{B}_{\varphi}^{+}$tal que $x=b_{-m}$. Além disso, reordene os monômios de maneira que:

i. caso $\prod_{\substack{i \in \mathbb{Z} \\ \varphi(i) \neq s g n(i)}} b_{i}^{\alpha_{i}} \prod_{\substack{j \in \mathbb{Z} \\ \varphi(i) \neq s g n(j)}}\left(b_{j}^{1}\right)^{\beta_{j}}$ possua como fator $b_{m}$, reescreva-o como $b_{m}^{\alpha_{m}} \prod_{\substack{i \in \mathbb{Z} \backslash\{m\} \\ \varphi(i) \neq s g n(i)}} b_{i}^{\alpha_{i}} \prod_{\substack{j \in \mathbb{Z} \\ \varphi(j) \neq s g n(j)}}\left(b_{j}^{1}\right)^{\beta_{j}}$ considerando as novas constantes e os novos monômios que surgirão desta mudança, como observado em (3.7).

ii. caso contrário, reescreva-o como $b_{i_{0}}^{\alpha_{i_{0}}} \prod_{\substack{i \in \mathbb{Z} \\ \varphi(i) \neq s g n(i)}} b_{i}^{\alpha_{i}} \prod_{\substack{j \in \mathbb{Z} \\ \varphi(j) \neq s g n(j)}}\left(b_{j}^{1}\right)^{\beta_{j}}, i_{0} \neq m$ é o primeiro fator à esquerda. 
Então, com as constantes apropriadas,

$$
\begin{aligned}
& w=\left(\sum_{\substack{i \in \mathbb{Z} \backslash\{0\} \\
\varphi(i) \neq \operatorname{sgn}(i)}} \xi_{i} b_{i}^{(n+1)}+\sum_{\substack{i \in \mathbb{Z} \backslash\{0\} \\
\varphi(i) \neq \operatorname{sgn}(i)}} \xi_{i}^{1}\left(b_{i}^{1}\right)^{(n+1)}\right. \\
& \left.+\sum_{(\bar{\alpha}, \bar{\beta})} \xi(\bar{\alpha}, \bar{\beta}) \prod_{\substack{i \in \mathbb{Z} \backslash\{0\} \\
\varphi(i) \neq \operatorname{sgn}(i)}} b_{i}^{\alpha_{i}} \prod_{\substack{i \in \mathbb{Z} \backslash\{0\} \\
\varphi(i) \neq \operatorname{sgn}(i)}}\left(b_{j}^{1}\right)^{\beta_{j}}\right) v \\
& =\left(\sum_{\substack{i \in \mathbb{Z} \backslash\{0\} \\
\varphi(i) \neq \operatorname{sgn}(i)}} \tilde{\xi}_{i} b_{i}^{(n+1)}+\sum_{\substack{i \in \mathbb{Z} \backslash\{0\} \\
\varphi(i) \neq \operatorname{sgn}(i)}} \tilde{\xi}_{i}^{1}\left(b_{i}^{1}\right)^{(n+1)}\right. \\
& +\sum_{(\bar{\alpha}, \bar{\beta})} \tilde{\xi}(\bar{\alpha}, \bar{\beta}) b_{m}^{\alpha_{m}} \prod_{\substack{i \in \mathbb{Z} \backslash\{0, m\} \\
\varphi(i) \neq s g n(i)}} b_{i}^{\alpha_{i}} \prod_{\substack{j \in \mathbb{Z} \backslash\{0\} \\
\varphi(j) \neq \operatorname{sgn}(j)}}\left(b_{j}^{1}\right)^{\beta_{j}} \\
& \left.+\sum_{(\bar{\alpha}, \bar{\beta})} \tilde{\xi}(\bar{\alpha}, \bar{\beta}) b_{i_{0}}^{\alpha_{i_{0}}} \prod_{\substack{i \in \mathbb{Z} \backslash\{0, m\} \\
\varphi(i) \neq \operatorname{sgn}(i)}} b_{i}^{\alpha_{i}} \prod_{\substack{j \in \mathbb{Z} \backslash\{0\} \\
\varphi(j) \neq s g n(j)}}\left(b_{j}^{1}\right)^{\beta_{j}}\right) v .
\end{aligned}
$$

Aplicando $x$ em $w$ obtemos

$$
\begin{aligned}
x w= & -\tilde{\xi}_{m} 2 \kappa_{0}(n+1) m b_{m}^{n} v+0+\sum_{(\bar{\alpha}, \bar{\beta})} \tilde{\xi}(\bar{\alpha}, \bar{\beta}) b_{-m} b_{m}^{\alpha_{m}} \prod_{i \neq m} b_{i}^{\alpha_{i}} \prod_{j<0}\left(b_{j}^{1}\right)^{\beta_{j}} v \\
& +\sum_{(\bar{\alpha}, \bar{\beta})} \tilde{\xi} b_{-m} b_{i_{0}}^{\alpha_{i_{0}}}(\bar{\alpha}, \bar{\beta}) b_{m}^{\alpha_{m}} \prod_{\substack{i \in \mathbb{Z} \backslash\{0, m\} \\
\varphi(i) \neq s g n(i)}} b_{i}^{\alpha_{i}} \prod_{\substack{j \in \mathbb{Z} \backslash\{0\} \\
\varphi(j) \neq s g n(j)}}\left(b_{j}^{1}\right)^{\beta_{j}} v \\
= & -\tilde{\xi}_{m} 2 \kappa_{0}(n+1) m b_{m}^{n} v \\
& +\sum_{(\bar{\alpha}, \bar{\beta})} \tilde{\xi}(\bar{\alpha}, \bar{\beta}) \alpha_{m}\left(2 m \kappa_{0}\right) b_{m}^{\alpha_{m}-1} \prod_{\substack{i \in \mathbb{Z} \backslash\{0, m\} \\
\varphi(i) \neq s g n(i)}} b_{i}^{\alpha_{i}} \prod_{\substack{j \in \mathbb{Z} \backslash\{0\} \\
\varphi(j) \neq s g n(j)}}\left(b_{j}^{1}\right)^{\beta_{j}} v .
\end{aligned}
$$

Portanto, $x w \neq 0$ e $\operatorname{deg}(x w)=n$. Pela hipótese de indução conseguimos reduzir $x w$ ao grau 0.

(b) Caso $\xi(\bar{\alpha}, \bar{\beta})=0, \forall(\bar{\alpha}, \bar{\beta})$.

i. Se existe $\xi_{k} \neq 0$ tome $x=b_{-k}$ e então $x w=2 \kappa_{0}(n+1) k \xi_{k}^{n} b_{k}^{n} v$.

ii. Se $\xi_{i}=0$ para todo $i \in \mathbb{Z}_{<0}$, tome $x=b_{-k}^{1}$, onde $k$ é o menor índice tal que $\xi_{k-1}^{1} \neq 0$. Teremos que $x w=4 \xi_{k-1}^{1} \kappa_{0}(n+1)\left(b_{k-1}^{1}\right) v$.

Portanto, $x w \neq 0$ e $\operatorname{deg}(x w)=n$.

Finalmente podemos afirmar que, por indução, dado um elemento arbitrário $w \in M_{\hat{\mathscr{B}}, \varphi}$ há um elemento $x \in \mathscr{B}_{\varphi}^{+}$tal que $x w \neq 0$ e $\operatorname{deg}(x w)=0$. Portanto, $M_{\hat{\mathscr{B}}, \varphi}$ não possui submódulos próprios. 
Suponha que $\kappa_{0}=0$. Considere o subconjunto $N_{\hat{\mathscr{B}}, \varphi}$ de $M_{\hat{\mathscr{B}}, \varphi}$ dado por

$$
N_{\hat{\mathscr{B}}, \varphi}=\bigoplus_{n \in \mathbb{Z} \backslash\{0\}}\left(M_{\hat{\mathscr{B}}}\right)_{n} .
$$

(i) De fato o subconjunto $N_{\hat{\mathscr{B}}, \varphi}$ é um submódulo de $M_{\hat{\mathscr{B}}, \varphi}$. Dados $x, y \in N_{\hat{\mathscr{B}}, \varphi}$ e $a, b \in$ $\mathfrak{U}(\hat{\mathscr{B}}),(x a+y b) \in N_{\hat{\mathscr{B}}, \varphi}$. Por consequência da Proposição 3.3.3 junta à hipótese de que $\kappa_{0}=0$, se $x \in \mathfrak{U}\left(\hat{\mathscr{B}}_{\varphi}^{+}\right)$então $x \cdot N_{\hat{\mathscr{B}}, \varphi}=0$, enquanto que se $y \in \mathfrak{U}\left(\hat{\mathscr{B}}_{\varphi}^{-}\right)$e $b \in N_{\hat{\mathscr{B}}, \varphi}$, $\operatorname{então} \operatorname{deg}(y b) \geq \operatorname{deg}(b)$.

(ii) Observe que $\sum_{i \in \mathbb{Z} \backslash\{0\}} \xi_{i} v \notin N_{\hat{\mathscr{B}}, \varphi}$, onde $\xi_{i} \in \mathbb{C}$ para todo $i \in \mathbb{Z} \backslash\{0\}$.

Por (i) e (ii) $N_{\hat{\mathscr{B}}, \varphi}$ é um submódulo próprio de $M_{\hat{\mathscr{B}}, \varphi}$.

\subsection{Módulos de Verma para $\hat{\mathscr{C}}$}

Novamente, fazendo o tensor da subálgebra de Cartan $\mathfrak{h}$ de $\mathfrak{g}$ com $R_{\mathscr{C}}$ obteremos uma subálgebra de Heisenberg da álgebra de Lie elíptica, que pode ser definida por meio de geradores e relações. A definição a seguir foi dada em [BCF09].

Definição 3.5.1. Considerando os geradores $b_{m}, b_{m}^{1}, m \in \mathbb{Z}, 1_{0}, 1_{ \pm}$, que podemos entender por $b_{m}=h \otimes t^{m}, b_{m}^{1}=h \otimes t^{m} u$ com $h \in \mathfrak{h}$ e $1_{0}, 1_{ \pm}$os elementos centrais que geram $\Omega_{R_{\mathscr{C}}}^{1} / d R_{\mathscr{C}}$. Esses geradores com as relações dadas por

$$
\begin{aligned}
& {\left[b_{m}, b_{n}\right]=2 n \delta_{m+n, 0} 1_{0},} \\
& {\left[b_{m}^{1}, b_{n}^{1}\right]=(n-m)\left(\delta_{m+n+2,-1}-2 b \delta_{m+n+2,0}+\delta_{m+n+2,1}\right) 1_{0}} \\
& {\left[b_{m}^{1}, b_{n}\right]=2 n\left(p_{|m+n+1|}(b) 1_{+}+q_{|m+n+1|}(b) 1_{-}\right),} \\
& {\left[b_{m}, 1_{0}\right]=\left[b_{m}^{1}, 1_{0}\right]=\left[b_{m}, 1_{ \pm}\right]=\left[b_{m}^{1}, 1_{ \pm}\right]=\left[1_{0}, 1_{ \pm}\right]=0,}
\end{aligned}
$$

resultam na subálgebra de Heisenberg de $\mathscr{C}$ denotada por $\hat{\mathscr{C}}$, e que chamaremos de álgebra de Heisenberg eliptica.

Note que caso $m, n \geq 0$ ou $m, n<-1$, teremos

$$
\left[b_{m}^{1}, b_{n}^{1}\right]=0 .
$$

Definamos os subespaços de $\hat{\mathscr{C}}$

$$
\hat{\mathscr{C}}^{ \pm}=\sum_{n \gtrless 0}\left(\mathbb{C} b_{n}+\mathbb{C} b_{n}^{1}\right) \text { e } \hat{\mathscr{C}}^{0}=\mathbb{C} 1_{-} \oplus \mathbb{C} 1_{0} \oplus \mathbb{C} 1_{+} \oplus \mathbb{C} b_{0} \oplus \mathbb{C} b_{-1}^{1}
$$

Agora observe a seguinte decomposição de $\hat{\mathscr{C}}$

$$
\hat{\mathscr{C}}=\hat{\mathscr{C}}^{-} \oplus \hat{\mathscr{C}}^{0} \oplus \hat{\mathscr{C}}^{+} .
$$

Neste ponto, considere a álgebra tipo de Borel $\mathfrak{b}_{\hat{\mathscr{C}}}=\hat{\mathscr{C}}^{0} \oplus \hat{\mathscr{C}}^{+}$. Podemos verificar por meio dos colchetes que, de fato, trata-se de uma álgebra.

Seja $\mathbb{C} v$ um $\mathfrak{b}_{\hat{\mathscr{C}}}$-módulo tal que $\hat{\mathscr{C}}^{+} v=0$ e para fixados $\lambda, \mu, \chi_{ \pm}, \kappa_{0} \in \mathbb{C}$,

$$
\begin{array}{ll}
b_{0} v=\lambda v \quad, \quad 1_{ \pm} v=\chi_{ \pm} v \\
b_{-1}^{1} v=\mu v \quad, \quad 1_{0} v=\kappa_{0} v
\end{array}
$$


Proposição 3.5.2. No $\mathfrak{b}_{\hat{\mathscr{C}}}$-módulo definido acima, temos

$$
\chi_{ \pm}=0
$$

Demonstração. Observe que podemos calcular utilizando a seção de Exemplos 2.5,

$$
\begin{array}{cc}
p_{2}(b)=\frac{4}{5}(b-1), & p_{3}(b)=\frac{1}{35}\left(32 b^{2}-48 b+11\right), \\
q_{2}(b)=\frac{1}{5}, & q_{3}(b)=\frac{4}{35}(2 b-1) .
\end{array}
$$

Então,

$$
\begin{aligned}
0 & =b_{0}^{1} b_{1} v-b_{1} b_{0}^{1} v=\left[b_{0}^{1}, b_{1}\right] v=2\left(p_{2}(b) 1_{+}+q_{2}(b) 1_{-}\right) v \\
& =2\left(\frac{4}{5}(b-1)\left(1_{+}\right)+\frac{1}{5}\left(1_{-}\right)\right) v \\
& \Leftrightarrow(4(b-1))\left(\chi_{+}\right)+\chi_{-}=0 .
\end{aligned}
$$

Além disso,

$$
\begin{aligned}
0 & =b_{1}^{1} b_{1} v-b_{1} b_{1}^{1} v=\left[b_{1}^{1}, b_{1}\right] v=2\left(p_{3}(b) 1_{+}+q_{3}(b) 1_{-}\right) v \\
& =2\left(\frac{1}{35}\left(32 b^{2}-48 b+11\right)\left(1_{+}\right)+\frac{4}{35}(2 b-1)\left(1_{-}\right)\right) v \\
& \Leftrightarrow\left(32 b^{2}-48 b+11\right)\left(\chi_{+}\right)+4(2 b-1)\left(\chi_{-}\right)=0 .
\end{aligned}
$$

Utilizando (3.55) em (3.5), obtemos que

$$
\begin{array}{r}
\left(32 b^{2}-48 b+11\right)\left(\chi_{+}\right)+4(2 b-1)\left(-(4(b-1))\left(\chi_{+}\right)=0\right. \\
\left(32 b^{2}-48 b+11\right)\left(\chi_{+}\right)+\left(-32 b^{2}+64 b-16\right)\left(\chi_{+}\right)=0 \\
-5\left(\chi_{+}\right)=0
\end{array}
$$

Logo, $\chi_{+}=0$ e consequentemente $\chi_{-}=0$.

Agora estamos prontos para induzir o módulo de Verma de $\hat{\mathscr{C}}$

$$
M_{\hat{\mathscr{C}}}:=\mathfrak{U}(\hat{\mathscr{C}}) \otimes_{\mathfrak{U}\left(\mathfrak{b}_{\hat{\mathscr{C}}}\right)} \mathbb{C} v
$$

Seja $w \in M_{\hat{\mathscr{C}}}=\mathfrak{U}(\hat{\mathscr{C}}) \otimes_{\mathfrak{U}\left(\mathfrak{b}_{\hat{\mathscr{C}}}\right)} \mathbb{C} v$. Pelo Teorema PBW (1.3.1), podemos descrever $M_{\hat{\mathscr{C}}}$ como um $\mathfrak{U}\left(\hat{\mathscr{C}}^{-}\right)$módulo livre. Munidos de raciocínio similar ao da Seção 3.1, descrevemos uma base ordenada de $\mathfrak{U}\left(\hat{\mathscr{C}}^{-}\right)$conveniente. Desta forma, podemos descrever um elemento arbitrário $w \in M_{\hat{\mathscr{C}}}$, como

$$
w=\sum_{(\bar{\alpha}, \bar{\beta})} \xi(\bar{\alpha}, \bar{\beta}) \prod_{i<0} b_{i}^{\alpha_{i}} \prod_{j<0}\left(b_{j}^{1}\right)^{\beta_{j}} v
$$

onde somente um número de $\xi(\bar{\alpha}, \bar{\beta}) \in \mathbb{C}$ é não nulo. Além disso, $\bar{\gamma}=\left\{\gamma_{-1}, \ldots, \gamma_{N}\right\}$ com $\gamma_{i} \in \mathbb{Z}_{>0}$ para todo $i \in \mathbb{Z}_{<0}$. 
Proposição 3.5.3. Se $m \in \mathbb{Z}_{>0}$ e $n \in \mathbb{Z}_{<0}$, teremos que

$$
\begin{aligned}
b_{m}\left(b_{n}\right)^{l} v & =\left(2 \ln \kappa_{0}\right) \delta_{m+n, 0}\left(\left(b_{n}\right)^{l-1}\right) v \\
b_{m}\left(b_{n}^{1}\right)^{l} v & =0 \\
b_{m}^{1}\left(b_{n}^{1}\right)^{l} v & =\left(\kappa_{0} l(n-m)\right)\left(\delta_{m+n+2,-1}-2 b \delta_{m+n+2,0}\right. \\
& \left.+\delta_{m+n+2,1}\right)\left(b_{n}^{1}\right)^{l-1} v, \\
b_{m}^{1}\left(b_{n}\right)^{l} v & =0 .
\end{aligned}
$$

Demonstração. A prova é dada pelo cálculo direto

$$
\begin{aligned}
b_{m}\left(b_{n}\right)^{l} v & =\left[b_{m},\left(b_{n}\right)^{l}\right] v+\left(b_{n}\right)^{l} b_{m} v \\
& =l\left(b_{n}\right)^{l-1}\left[b_{m}, b_{n}\right] v \\
& =\left(2 l n \kappa_{0}\right) \delta_{m+n, 0}\left(\left(b_{n}\right)^{l-1}\right) v . \\
b_{m}\left(b_{n}^{1}\right)^{l} v & =\left[b_{m},\left(b_{n}^{1}\right)^{l}\right] v+\left(b_{n}^{1}\right)^{l} b_{m} v \\
& =l\left(b_{n}^{1}\right)^{l-1}\left[b_{m}, b_{n}^{1}\right] v \\
& =0 \\
b_{m}^{1}\left(b_{n}^{1}\right)^{l} v & =\left[b_{m}^{1},\left(b_{n}^{1}\right)^{l}\right] v+\left(b_{n}^{1}\right)^{l} b_{m}^{1} v \\
& =l\left(b_{n}^{1}\right)^{l-1}\left[b_{m}^{1}, b_{n}^{1}\right] v \\
& =\left(\kappa_{0}^{l} l(n-m)\right)\left(\delta_{m+n+2,-1}-2 b \delta_{m+n+2,0}+\delta_{m+n+2,1}\right)\left(b_{n}^{1}\right)^{l-1} v . \\
b_{m}^{1}\left(b_{n}\right)^{l} & =\left[b_{m}^{1},\left(b_{n}\right)^{l}\right] v+\left(b_{n}\right)^{l} b_{m}^{1} v \\
& =l\left(b_{n}\right)^{l-1}\left[b_{m}^{1}, b_{n}\right] v \\
& =0 .
\end{aligned}
$$

Teorema 3.5.4. $M_{\hat{\mathscr{C}}}$ é irredutível se, e somente se, $\kappa_{0} \neq 0$.

Demonstração. Como trata-se de uma álgebra de Heisenberg, o módulo de Verma $M_{\hat{\mathscr{C}}}$ é $\mathbb{Z}$-graduado, de forma que

$$
M_{\hat{\mathscr{C}}}=\bigoplus_{n \in \mathbb{Z}}\left(M_{\hat{\mathscr{C}}}\right)_{n}
$$

onde

$$
\left(M_{\hat{\mathscr{C}}}\right)_{n}=\left\{w \in M_{\hat{\mathscr{C}}}: w=\sum_{(\bar{\alpha}, \bar{\beta})} \xi(\bar{\alpha}, \bar{\beta}) \prod_{i<0} b_{i}^{\alpha_{i}} \prod_{j<0}\left(b_{j}^{1}\right)^{\beta_{j}} v, \sum_{i} \alpha_{i}+\sum_{j} \beta_{j}=n, \forall(\bar{\alpha}, \bar{\beta})\right\} .
$$

A estratégia para demonstração deste teorema será similar à do Teorema 3.1.4 e Teorema 3.3.4, isto é, mostrar que assumindo a hipótese de que $\kappa_{0} \neq 0, M_{\hat{\mathscr{C}}}$ não possui submódulos próprios. Procederemos novamente por indução em $\operatorname{deg}(w)$.

1. Base de indução:

Suponha que $\operatorname{deg}(w)=1$, então

$$
w=\left(\sum_{i<0} \xi_{i} b_{i}+\sum_{i<0} \xi_{i}^{1} b_{i}^{1}\right) v
$$

onde somente um número de $\xi_{i}, \xi_{j}^{1} \in \mathbb{C}$ é diferente de zero. 
(a) Caso $\xi_{i}=0, \forall i \in \mathbb{Z}_{<0}$, então existe $\xi_{k}^{1} \neq 0$ para algum $k \in \mathbb{Z}_{<0}$. Seja $x \in \hat{\mathscr{C}}^{+}$tal que $x=b_{-k}^{1}$ e $k$ é o menor índice tal que $\xi_{k-3}^{1} \neq 0$. Teremos que

$$
x w=x \sum_{i<0} \xi_{i}^{1} b_{i}^{1} v=(2 k-3) \kappa_{0} \xi_{k-3}^{1} v
$$

(b) Caso exista $\xi_{k} \neq 0$ para $k \in \mathbb{Z}_{<0}$. Seja $x \in \hat{\mathscr{C}}^{+}$tal que $x=b_{-k}$. Teremos que

$$
x w=x \sum_{i<0} \xi_{i} b_{i} v=2 \kappa_{0} k \xi_{k} v .
$$

Portanto, em ambos os casos existe $x \in \hat{\mathscr{C}}^{+}$tal que $x w \neq 0$ e $\operatorname{deg}(x w)=0$.

2. Passo de indução: Suponha que para todo $w \in M_{\hat{\mathscr{C}}}$ com grau $n$, exista $y \in \mathscr{C}^{+}$tal que $y w \neq 0$ e $\operatorname{deg}(y w)=0$. Agora, $\operatorname{suponha}$ que $\operatorname{deg}(w)=n+1$, então

$$
w=\left(\sum_{i<0} \xi_{i} b_{i}^{(n+1)}+\sum_{i<0} \xi_{i}^{1}\left(b_{i}^{1}\right)^{(n+1)}+\sum_{(\bar{\alpha}, \bar{\beta})} \xi(\bar{\alpha}, \bar{\beta}) \prod_{i<0} b_{i}^{\alpha_{i}} \prod_{j<0}\left(b_{j}^{1}\right)^{\beta_{j}}\right) v
$$

onde somente um número de $\xi_{i}, \xi_{i}^{1}$ e $\xi(\bar{\alpha}, \bar{\beta})$ é diferente de zero.

(a) Caso exista $\xi(\bar{\alpha}, \bar{\beta}) \neq 0$, então tome $\xi(\bar{k}, \bar{l}) \neq 0$ e sejam $m$ o maior índice tal que $k_{m} \neq 0$ e $x \in \mathscr{C}^{+}$tal que $x=b_{-m}$. Além disso, reordene os monômios de maneira que:

i. caso $\prod_{i<0} b_{i}^{\alpha_{i}} \prod_{j<0}\left(b_{j}^{1}\right)^{\beta_{j}}$ possua como fator $b_{m}$, reescreva-o como $b_{m}^{\alpha_{m}} \prod_{i<0, i \neq m} b_{i}^{\alpha_{i}} \prod_{j<0}\left(b_{j}^{1}\right)^{\beta_{j}}$ considerando as novas constantes e os novos monômios que surgirão desta mudança, como observado em (3.7).

ii. caso contrário, reescreva-o como $b_{i_{0}}^{\alpha_{i_{0}}} \prod_{i<i_{0}} b_{i}^{\alpha_{i}} \prod_{j<0}\left(b_{j}^{1}\right)^{\beta_{j}}, i_{0} \neq m$ é o primeiro fator à esquerda.

Então, com as constantes apropriadas,

$$
\begin{aligned}
w= & \left(\sum_{i<0} \xi_{i} b_{i}^{(n+1)}+\sum_{i<0} \xi_{i}^{1}\left(b_{i}^{1}\right)^{(n+1)}+\sum_{(\bar{\alpha}, \bar{\beta})} \xi(\bar{\alpha}, \bar{\beta}) \prod_{i<0} b_{i}^{\alpha_{i}} \prod_{j<0}\left(b_{j}^{1}\right)^{\beta_{j}}\right) v \\
= & \left(\sum_{i<0} \tilde{\xi}_{i} b_{i}^{(n+1)}+\sum_{i<0} \tilde{\xi}_{i}^{1}\left(b_{i}^{1}\right)^{(n+1)}+\sum_{(\bar{\alpha}, \bar{\beta})} \tilde{\xi}(\bar{\alpha}, \bar{\beta}) b_{m}^{\alpha_{m}} \prod_{i \neq m} b_{i}^{\alpha_{i}} \prod_{j<0}\left(b_{j}^{1}\right)^{\beta_{j}}\right. \\
& \left.+\sum_{(\bar{\alpha}, \bar{\beta})} \tilde{\xi} b_{i_{0}}^{\alpha_{i_{0}}}(\bar{\alpha}, \bar{\beta}) b_{m}^{\alpha_{m}} \prod_{i \neq m} b_{i}^{\alpha_{i}} \prod_{j<0}\left(b_{j}^{1}\right)^{\beta_{j}}\right) v .
\end{aligned}
$$


Aplicando $x$ em $w$ obtemos

$$
\begin{aligned}
x w= & -\tilde{\xi}_{m} 2 \kappa_{0}(n+1) m b_{m}^{n} v+0+\sum_{(\bar{\alpha}, \bar{\beta})} \tilde{\xi}(\bar{\alpha}, \bar{\beta}) b_{-m} b_{m}^{\alpha_{m}} \prod_{i \neq m} b_{i}^{\alpha_{i}} \prod_{j<0}\left(b_{j}^{1}\right)^{\beta_{j}} v \\
& +\sum_{(\bar{\alpha}, \bar{\beta})} \tilde{\xi} b_{-m} b_{i_{0}}^{\alpha_{i_{0}}}(\bar{\alpha}, \bar{\beta}) b_{m}^{\alpha_{m}} \prod_{i \neq m} b_{i}^{\alpha_{i}} \prod_{j<0}\left(b_{j}^{1}\right)^{\beta_{j}} v \\
= & -\tilde{\xi}_{m} 2 \kappa_{0}(n+1) m b_{m}^{n} v \\
& +\sum_{(\bar{\alpha}, \bar{\beta})} \tilde{\xi}(\bar{\alpha}, \bar{\beta}) \alpha_{m}\left(2 m \kappa_{0}\right) b_{m}^{\alpha_{m}-1} \prod_{i \neq m} b_{i}^{\alpha_{i}} \prod_{j<0}\left(b_{j}^{1}\right)^{\beta_{j}} v .
\end{aligned}
$$

Portanto, $x w \neq 0$ e $\operatorname{deg}(x w)=n$. Pela hipótese de indução conseguimos reduzir $x w$ ao grau 0.

(b) Caso $\xi(\bar{\alpha}, \bar{\beta})=0, \forall(\bar{\alpha}, \bar{\beta})$.

i. Se existe $\xi_{k} \neq 0$ tome $x=b_{-k}$ e então $x w=2 \kappa_{0}(n+1) k \xi_{k}^{n} b_{k}^{n} v$.

ii. Se $\xi_{i}=0$ para todo $i \in \mathbb{Z}_{<0}$, tome $x=b_{-k}^{1}$, onde $k$ é o menor índice tal que $\xi_{k-3}^{1} \neq 0$. Teremos que $x w=4 \xi_{k-3}^{1} \kappa_{0}(n+1)\left(b_{k-3}^{1}\right) v$.

Portanto, $x w \neq 0$ e $\operatorname{deg}(x w)=n$.

Finalmente podemos afirmar que, por indução, dado um elemento arbitrário $w \in M_{\hat{\mathscr{C}}}$ há um elemento $x \in \mathscr{C}^{+}$tal que $x w \neq 0$ e $\operatorname{deg}(x w)=0$, isto é, que gera todo o módulo $M_{\hat{\mathscr{C}}}$. Portanto, $M_{\hat{\mathscr{C}}}$ não possui submódulos próprios; em outras palavras, é irredutível como queríamos demonstrar.

Suponha que $\kappa_{0}=0$. Considere o subconjunto $N_{\hat{\mathscr{C}}}$ de $M_{\hat{\mathscr{C}}}$ dado por

$$
N_{\hat{\mathscr{C}}}=\bigoplus_{n \in \mathbb{Z} \backslash\{0\}}\left(M_{\hat{\mathscr{C}}}\right)_{n}
$$

(i) De fato o subconjunto $N_{\hat{\mathscr{C}}}$ é um submódulo de $M_{\hat{\mathscr{C}}}$. Dados $x, y \in N_{\hat{\mathscr{C}}}$ e $a, b \in \mathfrak{U}(\hat{\mathscr{C}})$, $(x a+y b) \in N_{\mathscr{\mathscr { C }}}$. Por consequência da Proposição 3.3.3 junta à hipótese de que $\kappa_{0}=0$, se $x \in \mathfrak{U}\left(\hat{\mathscr{C}}^{+}\right)$então $x \cdot N_{\hat{\mathscr{C}}}=0$, enquanto que se $y \in \mathfrak{U}\left(\hat{\mathscr{C}}^{-}\right)$e $b \in N_{\hat{\mathscr{C}}}$, então $\operatorname{deg}(y b) \geq \operatorname{deg}(b)$.

(ii) Observe que $\sum_{i \in \mathbb{Z}} \xi_{i} v \notin N_{\hat{\mathscr{C}}}$, onde $\xi_{i} \in \mathbb{C}$ para todo $i \in \mathbb{Z}$.

Por (i) e (ii) $N_{\hat{\mathscr{C}}}$ é um submódulo próprio de $M_{\hat{\mathscr{C}}}$.

\subsection{Módulos $\varphi$-Verma para $\hat{\mathscr{C}}$}

Definamos os subespaços de $\hat{\mathscr{C}}$

$$
\hat{\mathscr{C}}_{\varphi}^{ \pm}=\left(\sum_{\substack{m \in \mathbb{Z}_{<0} \\ \varphi(n)=\mp}}\left(\mathbb{C} b_{n}+\mathbb{C} b_{n}^{1}\right)\right) \oplus\left(\sum_{\substack{m \in \mathbb{Z}>0 \\ \varphi(m)= \pm}}\left(\mathbb{C} b_{n}+\mathbb{C} b_{n}^{1}\right)\right) .
$$


Agora observe a seguinte decomposição de $\hat{\mathscr{C}}$

$$
\hat{\mathscr{C}}=\hat{\mathscr{C}}_{\varphi}^{-} \oplus \hat{\mathscr{C}}^{0} \oplus \hat{\mathscr{C}}_{\varphi}^{+} .
$$

Considere a álgebra tipo de Borel $\mathfrak{b}_{\mathscr{\mathscr { C }}, \varphi}=\hat{\mathscr{C}}^{0} \oplus \hat{\mathscr{C}}_{\varphi}^{+}$. Podemos verificar por meio dos colchetes que, de fato, trata-se de uma álgebra.

Seja $\mathbb{C} v$ um $\hat{\mathfrak{b}}_{\varphi}$-módulo tal que $\hat{\mathscr{C}}_{\varphi}^{+} \cdot v=0$ e para fixados $\lambda, \mu, \chi_{ \pm}, \kappa_{0} \in \mathbb{C}$

$$
\begin{array}{ll}
b_{0} \cdot v=\lambda v \quad, \quad 1_{ \pm} \cdot v=\chi_{ \pm} v \\
b_{-1}^{1} \cdot v=\mu v \quad, \quad 1_{0} \cdot v=\kappa_{0} v
\end{array}
$$

Proposição 3.6.1. No $\mathfrak{b}_{\hat{\mathscr{C}}, \varphi}$-módulo definido acima, temos

$$
\chi \pm=0 .
$$

Demonstração. Caso $\varphi(1)=-, \varphi(-1)=+$ e então

$$
\begin{aligned}
0 & =b_{0}^{1} b_{-1} v-b_{-1} b_{0}^{1} v=\left[b_{0}^{1}, b_{-1}\right] v=-2\left(p_{0}(b) 1_{+}+q_{0}(b) 1_{-}\right) v \\
& =-2 \chi_{-} v \\
& \Leftrightarrow \chi_{-}=0
\end{aligned}
$$

Além disso,

$$
\begin{aligned}
0 & =b_{-1}^{1} b_{-1} v-b_{-1} b_{-1}^{1} v=\left[b_{-1}^{1}, b_{-1}\right] v=-2\left(p_{1}(b) 1_{+}+q_{1}(b) 1_{-}\right) v \\
& =-2 \chi_{+} v \\
& \Leftrightarrow \chi_{+}=0
\end{aligned}
$$

Utilizando (3.67) e (3.68), obtemos que neste caso $\chi_{+}=\chi_{-}=0$.

Caso $\varphi(1)=+$, veja a demonstração 3.5.2.

Agora estamos prontos para introduzir o módulo $\varphi$-Verma de $\hat{\mathscr{C}}$

$$
M_{\hat{\mathscr{C}}, \varphi}=\mathfrak{U}(\hat{\mathscr{C}}) \otimes_{\mathfrak{b}_{\hat{\mathscr{C}} \varphi}} \mathbb{C} v
$$

Podemos descrever um elemento arbitrário $w \in M_{\hat{\mathscr{C}}, \varphi}$, como

$$
w=\left(\sum_{(\bar{\alpha}, \bar{\beta})} \xi(\bar{\alpha}, \bar{\beta}) \prod_{\substack{i \in \mathbb{Z} \\ \varphi(i) \neq \operatorname{sgn}(i)}} b_{i}^{\alpha_{i}} \prod_{\substack{j \in \mathbb{Z} \\ \varphi(j) \neq \operatorname{sgn}(j)}}\left(b_{j}^{1}\right)^{\beta_{j}}\right) v
$$

onde somente um número de $\xi(\bar{\alpha}, \bar{\beta}) \in \mathbb{C}$ é não nulo. Além disso, $\bar{\gamma}=\left\{\gamma_{N_{1}}, \ldots, \gamma_{N_{k}}\right\}$ com $\gamma_{i} \in \mathbb{Z}_{>0}$ para todo $i \in \mathbb{Z} \backslash\{0\}$. 
Proposição 3.6.2. Se $m, n \in \mathbb{Z} \backslash\{0\}, \varphi(m)=\operatorname{sgn}(m)$ e $\varphi(n) \neq \operatorname{sgn}(n)$, então

$$
\begin{aligned}
b_{m}\left(b_{n}\right)^{l} v & =\left(2 \ln \kappa_{0}\right) \delta_{m+n, 0}\left(\left(b_{n}\right)^{l-1}\right) v, \\
b_{m}\left(b_{n}^{1}\right)^{l} v & =0 \\
b_{m}^{1}\left(b_{n}^{1}\right)^{l} v & =\left(\kappa_{0} l(n-m)\right)\left(\delta_{m+n+2,-1}-2 b \delta_{m+n+2,0}\right. \\
& \left.+\delta_{m+n+2,1}\right)\left(b_{n}^{1}\right)^{l-1} v, \\
b_{m}^{1}\left(b_{n}\right)^{l} v & =0 .
\end{aligned}
$$

Demonstração. A prova é dada pelo cálculo direto

$$
\begin{aligned}
b_{m}\left(b_{n}\right)^{l} v & =\left[b_{m},\left(b_{n}\right)^{l}\right] v+\left(b_{n}\right)^{l} b_{m} v \\
& =l\left(b_{n}\right)^{l-1}\left[b_{m}, b_{n}\right] v \\
& =\left(2 l n \kappa_{0}\right) \delta_{m+n, 0}\left(\left(b_{n}\right)^{l-1}\right) v . \\
b_{m}\left(b_{n}^{1}\right)^{l} v & =\left[b_{m},\left(b_{n}^{1}\right)^{l}\right] v+\left(b_{n}^{1}\right)^{l} b_{m} v \\
& =l\left(b_{n}^{1}\right)^{l-1}\left[b_{m}, b_{n}^{1}\right] v \\
& =0 . \\
b_{m}^{1}\left(b_{n}^{1}\right)^{l} v & =\left[b_{m}^{1},\left(b_{n}^{1}\right)^{l}\right] v+\left(b_{n}^{1}\right)^{l} b_{m}^{1} v \\
& =l\left(b_{n}^{1}\right)^{l-1}\left[b_{m}^{1}, b_{n}^{1}\right] v \\
& =\left(\kappa_{0}^{l} l(n-m)\right)\left(\delta_{m+n+2,-1}-2 b \delta_{m+n+2,0}+\delta_{m+n+2,1}\right)\left(b_{n}^{1}\right)^{l-1} v . \\
b_{m}^{1}\left(b_{n}\right)^{l} & =\left[b_{m}^{1},\left(b_{n}\right)^{l}\right] v+\left(b_{n}\right)^{l} b_{m}^{1} v \\
& =l\left(b_{n}\right)^{l-1}\left[b_{m}^{1}, b_{n}\right] v \\
& =0 .
\end{aligned}
$$

Teorema 3.6.3. O módulo $\varphi$-Verma $M_{\hat{\mathscr{C}}, \varphi}$ é irredutível se, e somente se, $\kappa_{0} \neq 0$.

Demonstração. O módulo $\varphi$-Verma $M_{\hat{\mathscr{C}}, \varphi}$ é $\mathbb{Z}$-graduado, de forma que

$$
M_{\hat{\mathscr{C}}, \varphi}=\bigoplus_{n \in \mathbb{Z}}\left(M_{\hat{\mathscr{C}}, \varphi}\right)_{n}
$$

onde

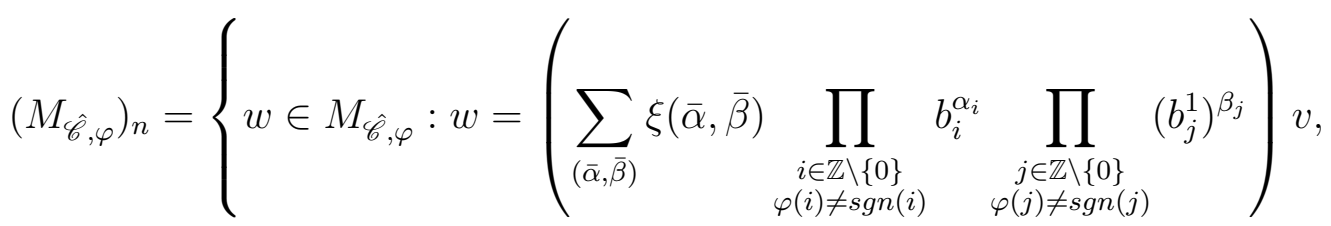

$$
\begin{aligned}
& \left.\sum_{i} \alpha_{i}+\sum_{j} \beta_{j}=n, \forall(\bar{\alpha}, \bar{\beta})\right\} \text {. }
\end{aligned}
$$

Procederemos novamente por indução em $\operatorname{deg}(w)$ para mostrar que todo elemento de $M_{\hat{\mathscr{C}}, \varphi}$ pode gerar o próprio módulo $M_{\hat{\mathscr{C}}, \varphi}$.

1. Base de indução: 
Suponha que $\operatorname{deg}(w)=1$, então

$$
w=\left(\sum_{\substack{i \in \mathbb{Z} \\ \varphi(i) \neq s g n(i)}} \xi_{i} b_{i}+\sum_{\substack{i \in \mathbb{Z} \\ \varphi(j) \neq s g n(j)}} \xi_{i}^{1} b_{i}^{1}\right) v
$$

onde somente um número de $\xi_{i}, \xi_{j}^{1} \in \mathbb{C}$ é diferente de zero.

(a) Caso $\xi_{i}=0, \forall i \in \mathbb{Z}_{<0}$, então existe $\xi_{k}^{1} \neq 0$ para algum $k \in \mathbb{Z}_{<0}$. Seja $x \in \hat{\mathscr{C}}_{\varphi}^{+}$tal que $x=b_{-k}^{1}$ e $k$ é o menor índice tal que $\xi_{k-3}^{1} \neq 0$. Teremos que

$$
x w=x \sum_{\substack{i \in \mathbb{Z} \\ \varphi(i) \neq \operatorname{sgn}(i)}} \xi_{i}^{1} b_{i}^{1} v=(2 k-3) \kappa_{0} \xi_{k-3}^{1} v .
$$

(b) Caso exista $\xi_{k} \neq 0$ para $k \in \mathbb{Z}_{<0}$. Seja $x \in \hat{\mathscr{C}}_{\varphi}^{+}$tal que $x=b_{-k}$. Teremos que

$$
x w=x \sum_{\substack{i \in \mathbb{Z} \\ \varphi(i) \neq \operatorname{sgn}(i)}} \xi_{i} b_{i} v=2 \kappa_{0} k \xi_{k} v .
$$

Portanto, em ambos os casos existe $x \in \hat{\mathscr{C}}_{\varphi}^{+}$tal que $x w \neq 0$ e $\operatorname{deg}(x w)=0$.

2. Passo de indução: Suponha que para todo $v \in M_{\hat{\mathscr{C}}, \varphi}$ com grau $n$, exista $y \in \mathscr{C}_{\varphi}^{+}$tal que $y w \neq 0$ e $\operatorname{deg}(y w)=0$. Agora, $\operatorname{suponha}$ que $\operatorname{deg}(w)=n+1$, então

$w=\left(\sum_{\substack{i \in \mathbb{Z} \\ \varphi(i) \neq s g n(i)}} \xi_{i} b_{i}^{(n+1)}+\sum_{\substack{i \in \mathbb{Z} \\ \varphi(i) \neq s g n(i)}} \xi_{i}^{1}\left(b_{i}^{1}\right)^{(n+1)}+\sum_{(\bar{\alpha}, \bar{\beta})} \xi(\bar{\alpha}, \bar{\beta}) \prod_{\substack{i \in \mathbb{Z} \\ \varphi(i) \neq s g n(i)}} b_{i}^{\alpha_{i}} \prod_{\substack{j \in \mathbb{Z} \\ \varphi(j) \neq \operatorname{sgn}(j)}}\left(b_{j}^{1}\right)^{\beta_{j}}\right) v$

onde somente um número de $\xi_{i}, \xi_{i}^{1}$ e $\xi(\bar{\alpha}, \bar{\beta})$ é diferente de zero. Reordene os monômios 
de maneira que possamos escrever com as constantes apropriadas,

$$
\begin{aligned}
& w=\left(\sum_{\substack{i \in \mathbb{Z} \backslash\{0\} \\
\varphi(i) \neq \operatorname{sgn}(i)}} \xi_{i} b_{i}^{(n+1)}+\sum_{\substack{i \in \mathbb{Z} \backslash\{0\} \\
\varphi(i) \neq \operatorname{sgn}(i)}} \xi_{i}^{1}\left(b_{i}^{1}\right)^{(n+1)}\right. \\
& \left.+\sum_{(\bar{\alpha}, \bar{\beta})} \xi(\bar{\alpha}, \bar{\beta}) \prod_{\substack{i \in \mathbb{Z} \backslash\{0\} \\
\varphi(i) \neq \operatorname{sgn}(i)}} b_{i}^{\alpha_{i}} \prod_{\substack{i \in \mathbb{Z} \backslash\{0\} \\
\varphi(i) \neq \operatorname{sgn}(i)}}\left(b_{j}^{1}\right)^{\beta_{j}}\right) v \\
& =\left(\sum_{\substack{i \in \mathbb{Z} \backslash\{0\} \\
\varphi(i) \neq \operatorname{sgn}(i)}} \tilde{\xi}_{i} b_{i}^{(n+1)}+\sum_{\substack{i \in \mathbb{Z} \backslash\{0\} \\
\varphi(i) \neq \operatorname{sgn}(i)}} \tilde{\xi}_{i}^{1}\left(b_{i}^{1}\right)^{(n+1)}\right. \\
& +\sum_{(\bar{\alpha}, \bar{\beta})} \tilde{\xi}(\bar{\alpha}, \bar{\beta}) b_{m}^{\alpha_{m}} \prod_{\substack{i \in \mathbb{Z} \backslash\{0, m\} \\
\varphi(i) \neq \operatorname{sgn}(i)}} b_{i}^{\alpha_{i}} \prod_{\substack{i \in \mathbb{Z} \backslash\{0\} \\
\varphi(i) \neq \operatorname{sgn}(i)}}\left(b_{j}^{1}\right)^{\beta_{j}} \\
& \left.+\sum_{(\bar{\alpha}, \bar{\beta})} \tilde{\xi}(\bar{\alpha}, \bar{\beta}) b_{i_{0}}^{\alpha_{i_{0}}} \prod_{\substack{i \in \mathbb{Z} \backslash\{0, m\} \\
\varphi(i) \neq s g n(i)}} b_{i}^{\alpha_{i}} \prod_{\substack{j \in \mathbb{Z} \backslash\{0\} \\
\varphi(j) \neq s g n(j)}}\left(b_{j}^{1}\right)^{\beta_{j}}\right) v .
\end{aligned}
$$

Aplicando $x$ em $w$ obtemos

$$
\begin{aligned}
x w= & -\tilde{\xi_{m}} 2 \kappa_{0}(n+1) m b_{m}^{n} v+0+\sum_{(\bar{\alpha}, \bar{\beta})} \tilde{\xi}(\bar{\alpha}, \bar{\beta}) b_{-m} b_{m}^{\alpha_{m}} \prod_{\substack{i \in \mathbb{Z} \backslash\{0, m\} \\
\varphi(i) \neq s g n(i)}} b_{i}^{\alpha_{i}} \prod_{\substack{j \in \mathbb{Z} \backslash\{0\} \\
\varphi(j) \neq s g n(j)}}\left(b_{j}^{1}\right)^{\beta_{j}} v \\
& +\sum_{(\bar{\alpha}, \bar{\beta})} \tilde{\xi} b_{-m} b_{i_{0}}^{\alpha_{i_{0}}}(\bar{\alpha}, \bar{\beta}) b_{m}^{\alpha_{m}} \prod_{\substack{i \in \mathbb{Z} \backslash\{0, m\} \\
\varphi(i) \neq s g n(i)}} b_{i}^{\alpha_{i}} \prod_{\substack{j \in \mathbb{Z} \backslash\{0\} \\
\varphi(j) \neq \operatorname{sgn}(j)}}\left(b_{j}^{1}\right)^{\beta_{j}} v \\
= & -\tilde{\xi_{m}} 2 \kappa_{0}(n+1) m b_{m}^{n} v \\
& +\sum_{(\bar{\alpha}, \bar{\beta})} \tilde{\xi}(\bar{\alpha}, \bar{\beta}) \alpha_{m}\left(2 m \kappa_{0}\right) b_{m}^{\alpha_{m}-1} \prod_{\substack{i \in \mathbb{Z} \backslash\{0, m\} \\
\varphi(i) \neq s g n(i)}} b_{i}^{\alpha_{i}} \prod_{\substack{j \in \mathbb{Z} \backslash\{0\} \\
\varphi(j) \neq s g n(j)}}\left(b_{j}^{1}\right)^{\beta_{j}} v .
\end{aligned}
$$

Portanto, $x w \neq 0$ e $\operatorname{deg}(x w)=n$. Pela hipótese de indução conseguimos reduzir $x w$ ao grau 0.

3. Caso $\xi(\bar{\alpha}, \bar{\beta})=0, \forall(\bar{\alpha}, \bar{\beta})$.

(a) Se existe $\xi_{k} \neq 0$ tome $x=b_{-k}$ e então $x w=2 \kappa_{0}(n+1) k \xi_{k}^{n} b_{k}^{n} v$.

(b) Se $\xi_{i}=0$ para todo $i \in \mathbb{Z}_{<0}$, tome $x=b_{-k}^{1}$, onde $k$ é o menor índice tal que $\xi_{k-3}^{1} \neq 0$. Teremos que $x w=4 \xi_{k-3}^{1} \kappa_{0}(n+1)\left(b_{k-3}^{1}\right) v$.

Portanto, $x w \neq 0$ e $\operatorname{deg}(x w)=n$.

Podemos afirmar que, por indução, dado um elemento arbitrário $w \in M_{\hat{\mathscr{C}}, \varphi}$ há um elemento $x \in \mathscr{C}_{\varphi}^{+}$tal que $x w \neq 0$ e $\operatorname{deg}(x w)=0$, isto é, que gera todo o módulo $M_{\mathscr{C}}$. Portanto, $M_{\hat{\mathscr{C}}, \varphi}$ não possui submódulos próprios; em outras palavras, é irredutível como queríamos demonstrar. 
Suponha que $\kappa_{0}=0$. Considere o subconjunto $N_{\hat{\mathscr{C}, \varphi}}$ de $M_{\hat{\mathscr{C}}, \varphi}$ dado por

$$
N_{\hat{\mathscr{C}}, \varphi}=\bigoplus_{n \in \mathbb{Z} \backslash\{0\}}\left(M_{\hat{\mathscr{C}}, \varphi}\right)_{n}
$$

(i) De fato o subconjunto $N_{\hat{\mathscr{C}}, \varphi}$ é um submódulo de $M_{\hat{\mathscr{C}}, \varphi}$. Dados $x, y \in N_{\hat{\mathscr{C}}, \varphi}$ e $a, b \in \mathfrak{U}(\hat{\mathscr{C}})$, $(x a+y b) \in N_{\mathscr{\mathscr { C }}}$. Por consequência da Proposição 3.6.2 junta à hipótese de que $\kappa_{0}=0$, se $x \in \mathfrak{U}\left(\hat{\mathscr{C}}_{\varphi}^{+}\right)$então $x \cdot N_{\hat{\mathscr{C}}, \varphi}=0$, enquanto que se $y \in \mathfrak{U}\left(\hat{\mathscr{C}}_{\varphi}^{-}\right)$e $b \in N_{\hat{\mathscr{C}}, \varphi}$, então $\operatorname{deg}(y b) \geq \operatorname{deg}(b)$.

(ii) Observe que $\sum_{i \in \mathbb{Z}} \xi_{i} v \notin N_{\hat{\mathscr{C}}, \varphi}$, onde $\xi_{i} \in \mathbb{C}$ para todo $i \in \mathbb{Z}$.

Por (i) e (ii) $N_{\hat{\mathscr{C}, \varphi}}$ é um submódulo próprio de $M_{\hat{\mathscr{C}}, \varphi}$.

\subsection{Módulos de Verma para $\hat{\mathscr{D}}$}

Novamente, se fizermos o tensor da subálgebra de Cartan $\mathfrak{h}$ de $\mathfrak{g}$ com $R_{\mathscr{D}}$, obteremos uma subálgebra de Heisenberg da álgebra de Lie DJKM que pode ser definida por meio de geradores e relações. Para definir esta álgebra, vamos necessitar da seguinte função polinomial:

$$
\psi_{i j}^{\prime}=\left\{\begin{array}{lll}
1_{i+j-2} & \text { para } i+j=1,0,-1,-2, \\
P_{-3, i+j-2}(c)\left(1_{-3}+c 1_{-1}\right) & \text { para } i+j=2 n-1 \geq 3, \\
P_{-3, i+j-2}(c)\left(c 1_{-3}+1_{-1}\right) & \text { para } i+j=-2 n+1 \leq-3, \\
P_{-4,|i+j|-2}(c) 1_{-4}+P_{-2,|i+j|-2}(c) 1_{-2} & \text { para }|i+j|=2 n \geq 2,
\end{array}\right.
$$

para $n \in \mathbb{Z}$ e $\psi^{\prime}(c, w)=\sum_{n \in \mathbb{Z}} \psi_{n}^{\prime}(c) w^{n}$ para $\psi_{i+j}^{\prime}(c):=\psi_{i j}^{\prime}(c)$.

A definição a seguir foi dada em [CFM14].

Definição 3.7.1. Considerando os geradores $b_{m}, b_{m}^{1}, m \in \mathbb{Z}, 1_{i}, i \in\{0,-1,-2,-3,-4\}$, que podemos entender por $b_{m}=h \otimes t^{m}, b_{m}^{1}=h \otimes t^{m} u$ com $h \in \mathfrak{h}$ e $1_{i}, i \in\{0,-1,-2,-3,-4\}$ os elementos centrais que geram $\Omega_{R_{\mathscr{D}}}^{1} / d R_{\mathscr{D}}$. Esses geradores com as relações dadas por

$$
\begin{aligned}
& {\left[b_{m}, b_{n}\right]=-2 m \delta_{m+n, 0} 1_{0},} \\
& {\left[b_{m}^{1}, b_{n}^{1}\right]=2\left((n+2) \delta_{m+n,-4}-2 c(n+1) \delta_{m+n,-2}+n \delta_{m+n, 0}\right) 1_{0},} \\
& {\left[b_{m}^{1}, b_{n}\right]=2 n \psi_{n m}^{\prime}(c),} \\
& {\left[b_{m}, 1_{i}\right]=\left[b_{m}^{1}, 1_{i}\right]=\left[1_{i}, 1_{j}\right]=0, i, j \in\{0,-1,-2,-3,-4\},}
\end{aligned}
$$

resultam na subálgebra de Heisenberg de $\mathscr{D}$ denotada por $\hat{\mathscr{D}}$, e que chamaremos de álgebra de Heisenberg DJKM.

Definamos os subespaços de $\hat{\mathscr{D}}$

$$
\hat{\mathscr{D}}^{ \pm}=\sum_{n \gtrless 0}\left(\mathbb{C} b_{n}+\mathbb{C} b_{n}^{1}\right) \text { e } \hat{\mathscr{D}}^{0}=\mathbb{C} 1_{0} \oplus \mathbb{C} 1_{-1} \oplus \mathbb{C} 1_{-2} \oplus \mathbb{C} 1_{-3} \oplus \mathbb{C} 1_{-4} \oplus \mathbb{C} b_{0} \oplus \mathbb{C} b_{0}^{1} .
$$

Agora observe a seguinte decomposição de $\hat{\mathscr{D}}$

$$
\hat{\mathscr{D}}=\hat{\mathscr{D}}^{-} \oplus \hat{\mathscr{D}}^{0} \oplus \hat{\mathscr{D}}^{+} .
$$


Neste ponto, considere a álgebra tipo de Borel $\mathfrak{b}_{\hat{\mathscr{D}}}=\hat{\mathscr{D}}^{0} \oplus \hat{\mathscr{D}}^{+}$. Podemos verificar por meio dos colchetes que, de fato, trata-se de uma álgebra.

Seja $\mathbb{C} v$ um $\mathfrak{b}_{\hat{\mathscr{D}}}$-módulo tal que $\hat{\mathscr{D}}^{+} v=0$ e para fixados $\lambda, \mu, \chi_{-1}, \chi_{-2}, \chi_{-3}, \chi_{-4}, \kappa_{0} \in \mathbb{C}$,

$$
\begin{array}{ll}
b_{0} v=\lambda v \quad, \quad 1_{j} v=\chi_{j} v, i \in\{-1,-2,-3,-4\}, \\
b_{0}^{1} v=\mu v \quad, \quad 1_{0} v=\kappa_{0} v .
\end{array}
$$

Proposição 3.7.2. No $\mathfrak{b}_{\hat{\mathscr{D}}}$-módulo definido acima, temos

$$
\chi_{-1}=\chi_{-2}=\chi_{-3}=\chi_{-4}=0 \text { e } \psi_{m n}^{\prime}=0 .
$$

Demonstração. Nesta demonstração, utilizaremos fortemente os cálculos obtidos em [CFT13] para determinados polinômios.

(i)

$$
0=b_{0}^{1} b_{1} v-b_{1} b_{0}^{1} v=\left[b_{0}^{1}, b_{1}\right] v=2 \psi_{01}^{\prime}(c) v=2 \chi_{-1} v .
$$

Portanto, $\chi_{-1}=0$.

(ii)

$$
0=b_{0}^{1} b_{-1} v-b_{-1} b_{0}^{1} v=\left[b_{0}^{1}, b_{-1}\right] v=-2 \psi_{0-1}^{\prime}(c) v=-2 \chi_{-3} v .
$$

Portanto, $\chi_{-3}=0$.

(iii)

$$
\begin{aligned}
0 & =b_{2}^{1} b_{2} v-b_{2} b_{2}^{1} v=\left[b_{2}^{1}, b_{2}\right] v=4 \psi_{22}^{\prime}(c) v=-4\left(P_{-4,2}(c) 1_{-4}+P_{-2,2}(c) 1_{-2}\right) \\
& =\frac{4}{5}\left(4 c 1_{-4}+1_{-2}\right)=0 \Leftrightarrow-4 c \chi_{-4}=\chi_{-2}
\end{aligned}
$$

e

$$
\begin{aligned}
0 & =b_{4}^{1} b_{4} v-b_{4} b_{4}^{1} v=\left[b_{4}^{1}, b_{4}\right] v=8 \psi_{44}^{\prime}(c) v=8\left(P_{-4,4}(c) 1_{-4}+P_{-2,4}(c) 1_{-2}\right) \\
& =\frac{1}{35}\left(\left(32 c^{2}-5\right) 1_{-4}+8\left(1_{-2}\right)\right) \Leftrightarrow\left(32 c^{2}-5\right) \chi_{-4}+8 c \chi_{-2}=0 .
\end{aligned}
$$

Portanto, $\chi_{-4}$ e consequentemente $\chi_{-2}=0$.

Finalmente obtemos que $\chi_{-1}=\chi_{-2}=\chi_{-3}=\chi_{-4}=0$ e $\psi_{m n}^{\prime}=0$.

Agora estamos prontos para induzir o módulo de Verma de $\hat{\mathscr{D}}$

$$
M_{\hat{\mathscr{D}}}:=\mathfrak{U}(\hat{\mathscr{D}}) \otimes_{\mathfrak{U}\left(\mathfrak{b}_{\hat{\mathscr{D}}}\right)} \mathbb{C} v
$$

Seja $w \in M_{\hat{\mathscr{D}}}=\mathfrak{U}(\hat{\mathscr{D}}) \otimes_{\mathfrak{U}\left(\mathfrak{b}_{\hat{\mathscr{D}}}\right)} \mathbb{C} v$. Podemos descrever um elemento arbitrário $w \in M_{\hat{\mathscr{D}}}$, como

$$
w=\sum_{(\bar{\alpha}, \bar{\beta})} \xi(\bar{\alpha}, \bar{\beta}) \prod_{i<0} b_{i}^{\alpha_{i}} \prod_{j<0}\left(b_{j}^{1}\right)^{\beta_{j}} v
$$

onde somente um número de $\xi(\bar{\alpha}, \bar{\beta}) \in \mathbb{C}$ é não nulo. Além disso, $\bar{\gamma}=\left\{\gamma_{-1}, \ldots, \gamma_{N}\right\}$ com $\gamma_{i} \in \mathbb{Z}_{>0}$ para todo $i \in \mathbb{Z}_{<0}$. 
Proposição 3.7.3. Se $m \in \mathbb{Z}_{>0}$ e $n \in \mathbb{Z}_{<0}$, teremos que

$$
\begin{aligned}
b_{m}\left(b_{n}\right)^{l} v & =\left(-2 l m \kappa_{0}\right) \delta_{m+n, 0}\left(\left(b_{n}\right)^{l-1}\right) v \\
b_{m}\left(b_{n}^{1}\right)^{l} v & =0 \\
b_{m}^{1}\left(b_{n}^{1}\right)^{l} v & =2 \kappa_{0} l\left((n+2) \delta_{m+n,-4}-2 c(n+1) \delta_{m+n,-2}+n \delta_{m+n, 0}\right) v \\
b_{m}^{1}\left(b_{n}\right)^{l} v & =0 .
\end{aligned}
$$

Demonstração. A prova é dada pelo cálculo direto

$$
\begin{aligned}
b_{m}\left(b_{n}\right)^{l} v & =\left[b_{m},\left(b_{n}\right)^{l}\right] v+\left(b_{n}\right)^{l} b_{m} v \\
& =l\left(b_{n}\right)^{l-1}\left[b_{m}, b_{n}\right] v \\
& =\left(-2 l m \kappa_{0}\right) \delta_{m+n, 0}\left(\left(b_{n}\right)^{l-1}\right) v . \\
b_{m}\left(b_{n}^{1}\right)^{l} v & =\left[b_{m},\left(b_{n}^{1}\right)^{l}\right] v+\left(b_{n}^{1}\right)^{l} b_{m} v \\
& =l\left(b_{n}^{1}\right)^{l-1}\left[b_{m}, b_{n}^{1}\right] v \\
& =0 . \\
b_{m}^{1}\left(b_{n}^{1}\right)^{l} v & =\left[b_{m}^{1},\left(b_{n}^{1}\right)^{l}\right] v+\left(b_{n}^{1}\right)^{l} b_{m}^{1} v \\
& =l\left(b_{n}^{1}\right)^{l-1}\left[b_{m}^{1}, b_{n}^{1}\right] v \\
& =2 \kappa_{0} l\left((n+2) \delta_{m+n,-4}-2 c(n+1) \delta_{m+n,-2}+n \delta_{m+n, 0}\right) v . \\
b_{m}^{1}\left(b_{n}\right)^{l} & =\left[b_{m}^{1},\left(b_{n}\right)^{l}\right] v+\left(b_{n}\right)^{l} b_{m}^{1} v \\
& =l\left(b_{n}\right)^{l-1}\left[b_{m}^{1}, b_{n}\right] v \\
& =0 .
\end{aligned}
$$

Teorema 3.7.4. O módulo de Verma $M_{\hat{\mathscr{D}}}$ é irredutível se, e somente se, $\kappa_{0} \neq 0$.

Demonstração. O módulo de Verma $M_{\hat{\mathscr{D}}}$ é $\mathbb{Z}$-graduado, de forma que

$$
M_{\hat{\mathscr{D}}}=\bigoplus_{n \in \mathbb{Z}}\left(M_{\hat{\mathscr{D}}}\right)_{n}
$$

onde

$$
\left(M_{\hat{\mathscr{D}}}\right)_{n}=\left\{w \in M_{\hat{\mathscr{D}}}: w=\sum_{(\bar{\alpha}, \bar{\beta})} \xi(\bar{\alpha}, \bar{\beta}) \prod_{i<0} b_{i}^{\alpha_{i}} \prod_{j<0}\left(b_{j}^{1}\right)^{\beta_{j}} v, \sum_{i} \alpha_{i}+\sum_{j} \beta_{j}=n, \forall(\bar{\alpha}, \bar{\beta})\right\} .
$$

Diremos que um elemento $w \in M_{\hat{\mathscr{D}}}$ tem grau $k$, denotando $\operatorname{deg}(w)=k$, se $w \in\left(M_{\hat{\mathscr{D}}}\right)_{k}$. A estratégia para demonstração deste teorema será similar à dos Teoremas anteriores, isto é, mostrar que assumindo a hipótese de que $\kappa_{0} \neq 0, M_{\hat{D}}$ não possui submódulos próprios. Procederemos novamente por indução em $\operatorname{deg}(w)$.

1. Base de indução:

Suponha que $\operatorname{deg}(w)=1$, então

$$
w=\left(\sum_{i<0} \xi_{i} b_{i}+\sum_{i<0} \xi_{i}^{1} b_{i}^{1}\right) v
$$

onde somente um número de $\xi_{i}, \xi_{j}^{1} \in \mathbb{C}$ é diferente de zero. 
(a) Caso $\xi_{i}=0, \forall i \in \mathbb{Z}_{<0}$, então existe $\xi_{k}^{1} \neq 0$ para algum $k \in \mathbb{Z}_{<0}$. Seja $x \in \hat{\mathscr{D}}^{+}$tal que $x=b_{-k}^{1}$ e $k$ é o maior índice tal que $\xi_{k-4}^{1} \neq 0$. Teremos que

$$
x w=x \sum_{i<0} \xi_{i}^{1} b_{i}^{1} v=2(k-2) \kappa_{0} \xi_{k-4}^{1} v .
$$

(b) Caso exista $\xi_{k} \neq 0$ para $k \in \mathbb{Z}_{<0}$. Seja $x \in \mathscr{D}^{+}$tal que $x=b_{-k}$. Teremos que

$$
x w=x \sum_{i<0} \xi_{i} b_{i} v=2 \kappa_{0} k \xi_{k} v
$$

Portanto, em ambos os casos existe $x \in \hat{\mathfrak{h}}_{3}^{+}$tal que $x w \neq 0$ e $\operatorname{deg}(x w)=0$.

2. Passo de indução: Suponha que para todo $w \in M_{\hat{\mathscr{B}}}$ com grau $n$, exista $y \in \hat{\mathscr{D}}^{+}$tal que $y w \neq 0$ e $\operatorname{deg}(y w)=0$. Agora, $\operatorname{suponha}$ que $\operatorname{deg}(w)=n+1$, então

$$
w=\left(\sum_{i<0} \xi_{i} b_{i}^{(n+1)}+\sum_{i<0} \xi_{i}^{1}\left(b_{i}^{1}\right)^{(n+1)}+\sum_{(\bar{\alpha}, \bar{\beta})} \xi(\bar{\alpha}, \bar{\beta}) \prod_{i<0} b_{i}^{\alpha_{i}} \prod_{j<0}\left(b_{j}^{1}\right)^{\beta_{j}}\right) v
$$

onde somente um número de $\xi_{i}, \xi_{i}^{1}$ e $\xi(\bar{\alpha}, \bar{\beta})$ é diferente de zero.

(a) Caso exista $\xi(\bar{\alpha}, \bar{\beta}) \neq 0$, então tome $\xi(\bar{k}, \bar{l}) \neq 0$ e sejam $m$ o maior índice tal que $k_{m} \neq 0$ e $x \in \mathscr{B}^{+}$tal que $x=b_{-m}$. Além disso, reordene os monômios de maneira que:

i. caso $\prod_{i<0} b_{i}^{\alpha_{i}} \prod_{j<0}\left(b_{j}^{1}\right)^{\beta_{j}}$ possua como fator $b_{m}$, reescreva-o como $b_{m}^{\alpha_{m}} \prod_{i<0, i \neq m}$ $b_{i}^{\alpha_{i}} \prod_{j<0}\left(b_{j}^{1}\right)^{\beta_{j}}$ considerando as novas constantes e os novos monômios que surgirão desta mudança, como observado em (3.7).

ii. caso contrário, reescreva-o como $b_{i_{0}}^{\alpha_{i_{0}}} \prod_{i<i_{0}} b_{i}^{\alpha_{i}} \prod_{j<0}\left(b_{j}^{1}\right)^{\beta_{j}}, i_{0} \neq m$ é o primeiro fator à esquerda.

Então, com as constantes apropriadas,

$$
\begin{aligned}
w= & \left(\sum_{i<0} \xi_{i} b_{i}^{(n+1)}+\sum_{i<0} \xi_{i}^{1}\left(b_{i}^{1}\right)^{(n+1)}+\sum_{(\bar{\alpha}, \bar{\beta})} \xi(\bar{\alpha}, \bar{\beta}) \prod_{i<0} b_{i}^{\alpha_{i}} \prod_{j<0}\left(b_{j}^{1}\right)^{\beta_{j}}\right) v \\
= & \left(\sum_{i<0} \tilde{\xi}_{i} b_{i}^{(n+1)}+\sum_{i<0} \tilde{\xi}_{i}^{1}\left(b_{i}^{1}\right)^{(n+1)}+\sum_{(\bar{\alpha}, \bar{\beta})} \tilde{\xi}(\bar{\alpha}, \bar{\beta}) b_{m}^{\alpha_{m}} \prod_{i \neq m} b_{i}^{\alpha_{i}} \prod_{j<0}\left(b_{j}^{1}\right)^{\beta_{j}}\right. \\
& \left.+\sum_{(\bar{\alpha}, \bar{\beta})} \tilde{\xi} b_{i_{0}}^{\alpha_{i}}(\bar{\alpha}, \bar{\beta}) b_{m}^{\alpha_{m}} \prod_{i \neq m} b_{i}^{\alpha_{i}} \prod_{j<0}\left(b_{j}^{1}\right)^{\beta_{j}}\right) v .
\end{aligned}
$$


Aplicando $x$ em $w$ obtemos

$$
\begin{aligned}
x w= & \tilde{\xi_{m}} 2 \kappa_{0}(n+1) m b_{m}^{n} v+0+\sum_{(\bar{\alpha}, \bar{\beta})} \tilde{\xi}(\bar{\alpha}, \bar{\beta}) b_{-m} b_{m}^{\alpha_{m}} \prod_{i \neq m} b_{i}^{\alpha_{i}} \prod_{j<0}\left(b_{j}^{1}\right)^{\beta_{j}} v \\
& +\sum_{(\bar{\alpha}, \bar{\beta})} \tilde{\xi} b_{-m} b_{i_{0}}^{\alpha_{i_{0}}}(\bar{\alpha}, \bar{\beta}) b_{m}^{\alpha_{m}} \prod_{i \neq m} b_{i}^{\alpha_{i}} \prod_{j<0}\left(b_{j}^{1}\right)^{\beta_{j}} v \\
= & -\tilde{\xi_{m}} 2 \kappa_{0}(n+1) m b_{m}^{n} v \\
& +\sum_{(\bar{\alpha}, \bar{\beta})} \tilde{\xi}(\bar{\alpha}, \bar{\beta}) \alpha_{m}\left(2 m \kappa_{0}\right) b_{m}^{\alpha_{m}-1} \prod_{i \neq m} b_{i}^{\alpha_{i}} \prod_{j<0}\left(b_{j}^{1}\right)^{\beta_{j}} v .
\end{aligned}
$$

Portanto, $x w \neq 0$ e $\operatorname{deg}(x w)=n$. Pela hipótese de indução conseguimos reduzir $x w$ ao grau 0.

(b) Caso $\xi(\bar{\alpha}, \bar{\beta})=0, \forall(\bar{\alpha}, \bar{\beta})$.

i. Se existe $\xi_{k} \neq 0$ tome $x=b_{-k}$ e então $x w=2 \kappa_{0} k(n+1) \xi_{k}^{n} b_{k}^{n} v$.

ii. Se $\xi_{i}=0$ para todo $i \in \mathbb{Z}_{<0}$, tome $x=b_{-k}^{1}$, onde $k$ é o maior índice tal que $\xi_{k-4}^{1} \neq 0$. Teremos que $x w=2 \xi_{k-4}^{1} \kappa_{0}(n+1)\left(b_{k-4}^{1}\right) v$.

Portanto, $x w \neq 0$ e $\operatorname{deg}(x w)=n$.

Finalmente podemos afirmar que, por indução, dado um elemento arbitrário $w \in M_{\hat{D}}$ há um elemento $x \in \hat{\mathscr{D}}^{+}$tal que $x w \neq 0$ e $\operatorname{deg}(x w)=0$, o que prova esta parte do teorema.

Suponha que $\kappa_{0}=0$. Considere o subconjunto $N_{\hat{\mathscr{D}}}$ de $M_{\hat{\mathscr{D}}}$ dado por

$$
N_{\hat{\mathscr{D}}}=\bigoplus_{n \in \mathbb{Z} \backslash\{0\}}\left(M_{\hat{\mathscr{D}}}\right)_{n}
$$

(i) De fato o subconjunto $N_{\hat{\mathscr{D}}}$ é um submódulo de $M_{\hat{\mathscr{D}}}$. Dados $x, y \in N_{\hat{\mathscr{D}}}$ e $a, b \in \mathfrak{U}(\hat{\mathscr{C}})$, $(x a+y b) \in N_{\hat{\mathscr{D}}}$. Por consequência da Proposição 3.8.2 junta à hipótese de que $\kappa_{0}=0$, se $x \in \mathfrak{U}\left(\hat{\mathscr{D}}^{+}\right)$então $x \cdot N_{\hat{\mathscr{D}}}=0$, enquanto que se $y \in \mathfrak{U}\left(\hat{\mathscr{D}}^{-}\right)$e $b \in N_{\hat{\mathscr{D}}}$, então $\operatorname{deg}(y b) \geq \operatorname{deg}(b)$.

(ii) Observe que $\sum_{i \in \mathbb{Z}} \xi_{i} v \notin N_{\hat{\mathscr{D}}}$, onde $\xi_{i} \in \mathbb{C}$ para todo $i \in \mathbb{Z}$.

Por (i) e (ii) $N_{\hat{D}}$ é um submódulo próprio de $M_{\hat{D}}$.

\subsection{Módulos $\varphi$-Verma para $\hat{\mathscr{D}}$}

Considere os subespaços de $\hat{\mathscr{D}}$

$$
\hat{\mathscr{D}}_{\varphi}^{ \pm}=\left(\sum_{\substack{m \in \mathbb{Z}_{<0} \\ \varphi(n)= \pm}}\left(\mathbb{C} b_{n}+\mathbb{C} b_{n}^{1}\right)\right) \oplus\left(\sum_{\substack{m \in \mathbb{Z}>0 \\ \varphi(m)=\mp}}\left(\mathbb{C} b_{n}+\mathbb{C} b_{n}^{1}\right)\right) .
$$

Agora observe a seguinte decomposição de $\hat{\mathscr{D}}$

$$
\hat{\mathscr{D}}=\hat{\mathscr{D}}_{\varphi}^{-} \oplus \hat{\mathscr{D}}^{0} \oplus \hat{\mathscr{D}}_{\varphi}^{+} .
$$


Considere a álgebra tipo de Borel $\mathfrak{b}_{\hat{D}, \varphi}=\hat{\mathscr{D}}^{0} \oplus \hat{\mathscr{D}}_{\varphi}^{+}$. Podemos verificar por meio dos colchetes que, de fato, trata-se de uma álgebra.

Seja $\mathbb{C} v$ um $\mathfrak{b}_{\hat{\mathscr{D}}, \varphi}$-módulo tal que $\hat{\mathscr{D}}_{\varphi}^{+} \cdot v=0$ e para fixados $\lambda, \mu, \chi_{-1}, \chi_{-2}, \chi_{-3}, \chi_{-4}, \kappa_{0} \in \mathbb{C}$,

$$
\begin{aligned}
& b_{0} \cdot v=\lambda v \quad, b_{0} \cdot v=\lambda v, \\
& b_{0}^{1} \cdot v=\mu v \quad, 1_{0} \cdot v=\kappa_{0} v . \\
& 1_{j} \cdot v=\chi_{j} v, i \in\{-1,-2,-3,-4\},
\end{aligned}
$$

Proposição 3.8.1. No $\mathfrak{b}_{\hat{\mathscr{D}}, \varphi}$-módulo definido acima, temos

$$
\chi_{-1}=\chi_{-2}=\chi_{-3}=\chi_{-4}=0 \text { e } \psi_{m n}^{\prime}=0 .
$$

Demonstração. Novamente utilizaremos os cálculos obtidos em [CFT13] para determinados polinômios.

(i)

$$
0=b_{0}^{1} b_{1} v-b_{1} b_{0}^{1} v=\left[b_{0}^{1}, b_{1}\right] v=2 \psi_{01}^{\prime}(c) v=2 \chi_{-1} v .
$$

Portanto, $\chi_{-1}=0$.

(ii)

$$
0=b_{0}^{1} b_{-1} v-b_{-1} b_{0}^{1} v=\left[b_{0}^{1}, b_{-1}\right] v=-2 \psi_{0-1}^{\prime}(c) v=-2 \chi_{-3} v .
$$

Portanto, $\chi_{-3}=0$.

(iii) Se $\varphi(2)=-$, então $\varphi(-2)=+$. Logo

$$
0=b_{0}^{1} b_{-2} v-b_{-2} b_{0}^{1} v=\left[b_{0}^{1}, b_{-2}\right] v=-4 \psi_{0-2}^{\prime}(c) v=-4 \chi_{-4} .
$$

Portanto, $\chi_{-4}=0$ e

$$
\begin{aligned}
0 & =b_{-2}^{1} b_{-2} v-b_{-2} b_{-2}^{1} v=\left[b_{-2}^{1}, b_{-2}\right] v=-8 \psi_{-2-2}^{\prime}(c) v=-8\left(P_{-2,2}(c) 1_{-2}\right) v \\
& =-8 \frac{1}{5} \chi_{-2}=0
\end{aligned}
$$

Portanto, $\chi_{-4}$ e $\chi_{-2}=0$.

(iv) Se $\varphi(2)=+$, veja a demonstração da Proposição 3.7.2.

Finalmente obtemos que $\chi_{-1}=\chi_{-2}=\chi_{-3}=\chi_{-4}=0$ e $\psi_{m n}^{\prime}=0$.

Agora estamos prontos para introduzir o módulo $\varphi$-Verma de $\hat{\mathscr{D}}$

$$
M_{\hat{\mathscr{D}}, \varphi}=\mathfrak{U}(\hat{\mathscr{D}}) \otimes_{\mathfrak{b}_{\hat{\mathscr{D}}, \varphi}} \mathbb{C} v
$$

Podemos descrever um elemento arbitrário $w \in M_{\hat{\mathscr{D}}, \varphi}$, como

$$
w=\left(\sum_{(\bar{\alpha}, \bar{\beta})} \xi(\bar{\alpha}, \bar{\beta}) \prod_{\substack{i \in \mathbb{Z} \\ \varphi(i) \neq s g n(i)}} b_{i}^{\alpha_{i}} \prod_{\substack{j \in \mathbb{Z} \\ \varphi(j) \neq s g n(j)}}\left(b_{j}^{1}\right)^{\beta_{j}}\right) v
$$

onde somente um número de $\xi(\bar{\alpha}, \bar{\beta}) \in \mathbb{C}$ é não nulo. Além disso, $\bar{\gamma}=\left\{\gamma_{-1}, \ldots, \gamma_{N}\right\}$ com $\gamma_{i} \in \mathbb{Z}_{>0}$ para todo $i \in \mathbb{Z}_{<0}$. 
Proposição 3.8.2. Se $m, n \in \mathbb{Z} \backslash\{0\}, \varphi(m)=\operatorname{sgn}(m)$ e $\varphi(n) \neq \operatorname{sgn}(n)$, então

$$
\begin{aligned}
& b_{m}\left(b_{n}\right)^{l} v=\left(-2 l m \kappa_{0}\right) \delta_{m+n, 0}\left(\left(b_{n}\right)^{l-1}\right) v, \\
& b_{m}\left(b_{n}^{1}\right)^{l} v=0 \\
& b_{m}^{1}\left(b_{n}^{1}\right)^{l} v=2 \kappa_{0} l\left((n+2) \delta_{m+n,-4}-2 c(n+1) \delta_{m+n,-2}+n \delta_{m+n, 0}\right) v, \\
& b_{m}^{1}\left(b_{n}\right)^{l} v=0 .
\end{aligned}
$$

Demonstração. A prova é dada pelo cálculo direto

$$
\begin{aligned}
b_{m}\left(b_{n}\right)^{l} v & =\left[b_{m},\left(b_{n}\right)^{l}\right] v+\left(b_{n}\right)^{l} b_{m} v \\
& =l\left(b_{n}\right)^{l-1}\left[b_{m}, b_{n}\right] v \\
& =\left(-2 l m \kappa_{0}\right) \delta_{m+n, 0}\left(\left(b_{n}\right)^{l-1}\right) v . \\
b_{m}\left(b_{n}^{1}\right)^{l} v & =\left[b_{m},\left(b_{n}^{1}\right)^{l}\right] v+\left(b_{n}^{1}\right)^{l} b_{m} v \\
& =l\left(b_{n}^{1}\right)^{l-1}\left[b_{m}, b_{n}^{1}\right] v \\
& =0 . \\
b_{m}^{1}\left(b_{n}^{1}\right)^{l} v & =\left[b_{m}^{1},\left(b_{n}^{1}\right)^{l}\right] v+\left(b_{n}^{1}\right)^{l} b_{m}^{1} v \\
& =l\left(b_{n}^{1}\right)^{l-1}\left[b_{m}^{1}, b_{n}^{1}\right] v \\
& =2 \kappa_{0} l\left((n+2) \delta_{m+n,-4}-2 c(n+1) \delta_{m+n,-2}+n \delta_{m+n, 0}\right) v . \\
b_{m}^{1}\left(b_{n}\right)^{l} & =\left[b_{m}^{1},\left(b_{n}\right)^{l}\right] v+\left(b_{n}\right)^{l} b_{m}^{1} v \\
& =l\left(b_{n}\right)^{l-1}\left[b_{m}^{1}, b_{n}\right] v \\
& =0 .
\end{aligned}
$$

Teorema 3.8.3. O módulo $\varphi$-Verma $M_{\hat{\mathscr{D}}, \varphi}$ é irredutível se, e somente se, $\kappa_{0} \neq 0$.

Demonstração. O módulo de Verma $M_{\hat{\mathscr{D}}, \varphi}$ é $\mathbb{Z}$-graduado, de forma que

$$
M_{\hat{\mathscr{D}}, \varphi}=\bigoplus_{n \in \mathbb{Z}}\left(M_{\hat{\mathscr{D}}, \varphi}\right)_{n}
$$

onde

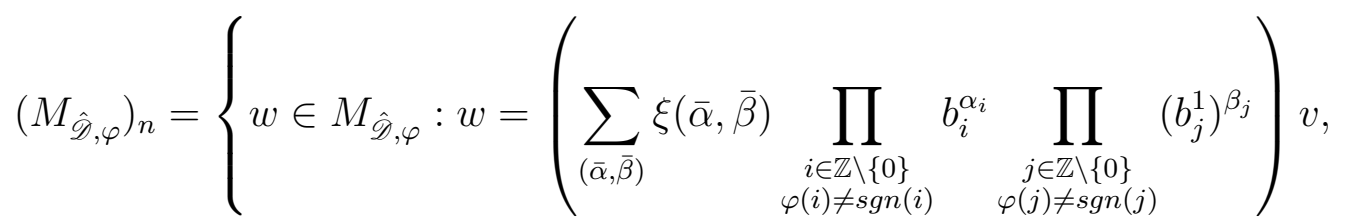

$$
\begin{aligned}
& \left.\sum_{i} \alpha_{i}+\sum_{j} \beta_{j}=n, \forall(\bar{\alpha}, \bar{\beta})\right\} .
\end{aligned}
$$

A estratégia para demonstração deste teorema será similar à dos Teoremas anteriores, isto é, mostrar que assumindo a hipótese de que $\kappa_{0} \neq 0, M_{\hat{D}}$ não possui submódulos próprios. Procederemos novamente por indução em $\operatorname{deg}(w)$. 
1. Base de indução: $\operatorname{Suponha} q u e \operatorname{deg}(w)=1$, então

$$
w=\left(\sum_{\substack{i \in \mathbb{Z} \backslash\{0\} \\ \varphi(i) \neq \operatorname{sgn}(i)}} \xi_{i} b_{i}+\sum_{\substack{i \in \mathbb{Z} \backslash\{0\} \\ \varphi(i) \neq \operatorname{sgn}(i)}} \xi_{i}^{1} b_{i}^{1}\right) v
$$

onde somente um número de $\xi_{i}, \xi_{i}^{1} \in \mathbb{C}$ é diferente de zero.

(a) Caso $\xi_{i}=0, \forall i \in \mathbb{Z}_{<0}$, então existe $\xi_{k}^{1} \neq 0$ para algum $k \in \mathbb{Z}_{<0}$. Seja $x \in \hat{\mathscr{D}}_{\varphi}^{+}$tal que $x=b_{-k}^{1}$ e $k$ é o maior índice tal que $\xi_{k-4}^{1} \neq 0$. Teremos que

$$
x w=x \sum_{\substack{i \in \mathbb{Z} \backslash\{0\} \\ \varphi(i) \neq s g n(i)}} \xi_{i}^{1} b_{i}^{1} v=2(k-2) \kappa_{0} \xi_{k-4}^{1} v .
$$

(b) Caso exista $\xi_{k} \neq 0$ para $k \in \mathbb{Z}_{<0}$. Seja $x \in \mathscr{D}_{\varphi}^{+}$tal que $x=b_{-k}$. Teremos que

$$
x w=x \sum_{\substack{i \in \mathbb{Z} \backslash\{0\} \\ \varphi(i) \neq \operatorname{sgn}(i)}} \xi_{i} b_{i} v=2 \kappa_{0} k \xi_{k} v .
$$

Portanto, em ambos os casos existe $x \in \mathscr{D}_{\varphi}^{+}$tal que $x w \neq 0$ e $\operatorname{deg}(x w)=0$.

2. Passo de indução: Suponha que para todo $v \in M_{\hat{\mathscr{B}}}$ com grau $n$, exista $y \in \hat{\mathscr{D}}^{+}$tal que $y w \neq 0$ e $\operatorname{deg}(y w)=0$. Agora, $\operatorname{suponha}$ que $\operatorname{deg}(w)=n+1$, então

$$
w=\left(\sum_{\substack{i \in \mathbb{Z} \backslash\{0\} \\ \varphi(i) \neq \operatorname{sgn}(i)}} \xi_{i} b_{i}^{(n+1)}+\sum_{\substack{i \in \mathbb{Z} \backslash\{0\} \\ \varphi(i) \neq \operatorname{sgn}(i)}} \xi_{i}^{1}\left(b_{i}^{1}\right)^{(n+1)}+\sum_{(\bar{\alpha}, \bar{\beta})} \xi(\bar{\alpha}, \bar{\beta}) \prod_{\substack{i \in \mathbb{Z} \backslash\{0\} \\ \varphi(i) \neq \operatorname{sgn}(i)}} b_{i}^{\alpha_{i}} \prod_{\substack{j \in \mathbb{Z} \backslash\{0\} \\ \varphi(j) \neq \operatorname{sgn}(j)}}\left(b_{j}^{1}\right)^{\beta_{j}}\right) v
$$

onde somente um número de $\xi_{i}, \xi_{i}^{1}$ e $\xi(\bar{\alpha}, \bar{\beta})$ é diferente de zero. Reordene os monômios 
de maneira que possamos escrever com as constantes apropriadas,

$$
\begin{aligned}
& w=\left(\sum_{\substack{i \in \mathbb{Z} \backslash\{0\} \\
\varphi(i) \neq \operatorname{sgn}(i)}} \xi_{i} b_{i}^{(n+1)}+\sum_{\substack{i \in \mathbb{Z} \backslash\{0\} \\
\varphi(i) \neq \operatorname{sgn}(i)}} \xi_{i}^{1}\left(b_{i}^{1}\right)^{(n+1)}\right. \\
& \left.+\sum_{(\bar{\alpha}, \bar{\beta})} \xi(\bar{\alpha}, \bar{\beta}) \prod_{\substack{i \in \mathbb{Z} \backslash\{0\} \\
\varphi(i) \neq \operatorname{sgn}(i)}} b_{i}^{\alpha_{i}} \prod_{\substack{j \in \mathbb{Z} \backslash\{0\} \\
\varphi(j) \neq \operatorname{sgn}(j)}}\left(b_{j}^{1}\right)^{\beta_{j}}\right) v \\
& =\left(\sum_{\substack{i \in \mathbb{Z} \backslash\{0\} \\
\varphi(i) \neq \operatorname{sgn}(i)}} \tilde{\xi}_{i} b_{i}^{(n+1)}+\sum_{\substack{i \in \mathbb{Z} \backslash\{0\} \\
\varphi(i) \neq \operatorname{sgn}(i)}} \tilde{\xi}_{i}^{1}\left(b_{i}^{1}\right)^{(n+1)}\right. \\
& +\sum_{(\bar{\alpha}, \bar{\beta})} \tilde{\xi}(\bar{\alpha}, \bar{\beta}) b_{m}^{\alpha_{m}} \prod_{i \neq m} b_{i}^{\alpha_{i}} \prod_{\substack{j \in \mathbb{Z} \backslash\{0\} \\
\varphi(j) \neq s g n(j)}}\left(b_{j}^{1}\right)^{\beta_{j}} \\
& \left.+\sum_{(\bar{\alpha}, \bar{\beta})} \tilde{\xi} b_{i_{0}}^{\alpha_{i_{0}}}(\bar{\alpha}, \bar{\beta}) b_{m}^{\alpha_{m}} \prod_{i \neq m} b_{i}^{\alpha_{i}} \prod_{\substack{j \in \mathbb{Z} \backslash\{0\} \\
\varphi(j) \neq \operatorname{sgn}(j)}}\left(b_{j}^{1}\right)^{\beta_{j}}\right) v .
\end{aligned}
$$

Aplicando $x$ em $w$ obtemos

$$
\begin{aligned}
x w= & \tilde{\xi}_{m} 2 \kappa_{0}(n+1) m b_{m}^{n} v+0+\sum_{(\bar{\alpha}, \bar{\beta})} \tilde{\xi}(\bar{\alpha}, \bar{\beta}) b_{-m} b_{m}^{\alpha_{m}} \prod_{i \neq m} b_{i}^{\alpha_{i}} \prod_{\substack{j \in \mathbb{Z} \backslash\{0\} \\
\varphi(j) \neq s g n(j)}}\left(b_{j}^{1}\right)^{\beta_{j}} v \\
& +\sum_{(\bar{\alpha}, \bar{\beta})} \tilde{\xi} b_{-m} b_{i_{0}}^{\alpha_{i_{0}}}(\bar{\alpha}, \bar{\beta}) b_{m}^{\alpha_{m}} \prod_{i \neq m} b_{i}^{\alpha_{i}} \prod_{\substack{j \in \mathbb{Z} \backslash\{0\} \\
\varphi(j) \neq s g n(j)}}\left(b_{j}^{1}\right)^{\beta_{j}} v \\
= & -\tilde{\xi}_{m} 2 \kappa_{0}(n+1) m b_{m}^{n} v \\
& +\sum_{(\bar{\alpha}, \bar{\beta})} \tilde{\xi}(\bar{\alpha}, \bar{\beta}) \alpha_{m}\left(2 m \kappa_{0}\right) b_{m}^{\alpha_{m}-1} \prod_{i \neq m} b_{i}^{\alpha_{i}} \prod_{\substack{j \in \mathbb{Z} \backslash\{0\} \\
\varphi(j) \neq s g n(j)}}\left(b_{j}^{1}\right)^{\beta_{j}} v .
\end{aligned}
$$

Portanto, $x w \neq 0$ e $\operatorname{deg}(x w)=n$. Pela hipótese de indução conseguimos reduzir $x w$ ao grau 0.

3. Caso $\xi(\bar{\alpha}, \bar{\beta})=0, \forall(\bar{\alpha}, \bar{\beta})$.

(a) Se existe $\xi_{k} \neq 0$ tome $x=b_{-k}$ e então $x w=2 \kappa_{0} k(n+1) \xi_{k}^{n} b_{k}^{n} v$.

(b) Se $\xi_{i}=0$ para todo $i \in \mathbb{Z}_{<0}$, tome $x=b_{-k}^{1}$, onde $k$ é o maior índice tal que $\xi_{k-4}^{1} \neq 0$. Teremos que $x w=2 \xi_{k-4}^{1} \kappa_{0}(n+1)\left(b_{k-4}^{1}\right) v$.

Portanto, $x w \neq 0$ e $\operatorname{deg}(x w)=n$.

Finalmente podemos afirmar que, por indução, dado um elemento arbitrário $w \in M_{\hat{\mathscr{D}}, \varphi}$ há um elemento $x \in \hat{\mathscr{D}}_{\varphi}^{+}$tal que $x w \neq 0$ e $\operatorname{deg}(x w)=0$, o que prova esta parte do teorema. 
Suponha que $\kappa_{0}=0$. Considere o subconjunto $N_{\hat{\mathscr{D}}, \varphi}$ de $M_{\hat{\mathscr{D}}, \varphi}$ dado por

$$
N_{\hat{\mathscr{D}}, \varphi}=\bigoplus_{n \in \mathbb{Z} \backslash\{0\}}\left(M_{\hat{\mathscr{D}}, \varphi}\right)_{n}
$$

(i) De fato o subconjunto $N_{\hat{\mathscr{D}}, \varphi}$ é um submódulo de $M_{\hat{\mathscr{D}}, \varphi}$. Dados $x, y \in N_{\hat{\mathscr{D}}, \varphi, \varphi}$ e $a, b \in$ $\mathfrak{U}(\hat{\mathscr{D}}),(x a+y b) \in N_{\hat{\mathscr{D}}}$. Por consequência da Proposição 3.8.2 junta à hipótese de que $\kappa_{0}=0$, se $x \in \mathfrak{U}\left(\hat{\mathscr{D}}_{\varphi}^{+}\right)$então $x \cdot N_{\hat{\mathscr{D}}, \varphi}=0$, enquanto que se $y \in \mathfrak{U}\left(\hat{\mathscr{D}}_{\varphi}^{-}\right)$e $b \in N_{\hat{\mathscr{D}}}$, então $\operatorname{deg}(y b) \geq \operatorname{deg}(b)$.

(ii) Observe que $\sum_{i \in \mathbb{Z}} \xi_{i} v \notin N_{\hat{\mathscr{D}}, \varphi}$, onde $\xi_{i} \in \mathbb{C}$ para todo $i \in \mathbb{Z}$.

Por (i) e (ii) $N_{\mathscr{\mathscr { D }}, \varphi}$ é um submódulo próprio de $M_{\hat{\mathscr{D}}, \varphi}$. 


\section{Referências Bibliográficas}

[BBFK13] Viktor Bekkert, Georgia Benkart, Vyacheslav Futorny e Iryna Kashuba. New irreducible modules for Heisenberg and affine Lie algebras. Journal of Algebra, 373:284-298, 2013. 1, 2, 3, 5, 11, 12, 25

[BCF09] Andre Bueno, Ben Cox e Vyacheslav Futorny. Free Field realizations of the Elliptic Affine Lie Algebra $\mathfrak{s l}(2, R) \oplus d \omega_{R} / d R$. Journal of Geometry and Physics, 59(9), 02 2009. iii, v, 1, 3, 21, 22, 43

[Bre95] Murray Bremner. Four-point affine Lie algebras. Proceedings of the American Mathematical Society, 123(7):1981-1989, 1995. iii, v, 1, 2, 3, 18, 19, 20

[CFM14] Ben Cox, Vyacheslav Futorny e Renato Alessandro Martins. Free field realizations of the Date-Jimbo-Kashiwara-Miwa algebra, volume 38 of Developments in Mathematics. Springer International Publishing, 2014. iii, v, 1, 3, 23, 24, 52

[CFT13] Ben Cox, Vyacheslav Futorny e Juan A. Tirao. DJKM algebras and non-classical orthogonal polynomials. Journal of Differential Equations, 255(9):2846-2870, 2013. 53, 57

[CJ13] Ben L. Cox e Elizabeth G. Jurisich. Realizations of the three point algebra $\mathfrak{s l}(2, R) \oplus\left(\omega_{R} / d R\right)$. Pacific Journal of Mathematics, 270(1):27-48, 2013. iii, v, $1,3,20,21,35$

[Cox08] Ben Cox. Realizations of the four point affine Lie algebra $\mathfrak{s l}(2, R) \oplus\left(\omega_{R} / d R\right)$. Pacific Journal of Mathematics, 234(2):260-288, 2008. iii, v, 1, 3, 26

[DKBTK97] Eddy A. De Kerf, Gerard G.A. Bäuerle e A.P.E. Ten Kroode. Lie Algebras, Part 2: Finite and Infinite Dimensional Lie Algebras and Applications in Physics, volume 7 of Studies in Mathematical Physics. Elsevier, 1997. 2, 8

[EGH+11] Pavel Etingof, Oleg Golberg, Sebastian Hensel, Tiankai Liu, Alex Schwendner, Dmitry Vaintrob e Elena Yudovina. Introduction to representation theory, volume 59 of Student Mathematical Library. American Mathematical Society, Providence, RI, 2011. With historical interludes by Slava Gerovitch. 5

[Fuc95] Jürgen Fuchs. Affine Lie algebras and quantum groups: An Introduction, with applications in conformal field theory. Cambridge university press, 1995. 2, 5

[Hum72] James E Humphreys. Introduction to Lie algebras and Representation Theory, volume 9 of Graduate Texts in Mathematics. Springer New York, 1972. 5

[Kac94] Victor G Kac. Infinite-dimensional Lie algebras, volume 44 of Progress in Mathematics. Cambridge university press, 1994. 3, 5, 11, 12 
[Kas84] Christian Kassel. Kähler differentials and coverings of complex simple Lie algebras extended over a commutative algebra. Journal of Pure and Applied Algebra, 34(2):265-275, 1984. 1, 19

[KN87] Igor Moiseevich Krichever e Sergei Petrovich Novikov. Algebras of Virasoro type, Riemann surfaces and structures of the theory of solutons. Functional Analysis and Its Applications, 21(2):126-142, 1987. 1, 17, 18

[MP95] Robert V Moody e Arturo Pianzola. Lie algebras with triangular decompositions. Wiley, 1995. 3, 6, 7, 11, 15

[Sch14] Martin Schlichenmaier. Krichever-Novikov Type Algebras Theory and Applications. Walter de Gruyter GmbH, 2014. 3

[SM10] Luiz Antonio Barrera San Martin. Algebras de Lie. Unicamp, 2010. 5

[SW16] Elias M. Stein e Guido Weiss. Introduction to Fourier analysis on Euclidean spaces (PMS-32), volume 32 of Princeton Mathematical Series. Princeton University Press, 2016. 19

[Sze39] Gabor Szeg. Orthogonal polynomials, volume 39 of Colloquium publications. American Mathematical Soc., 1939. 19 